\title{
Layering transitions, disordered flat phases, reconstruction, and roughening
}

\author{
Anoop Prasad and Peter B. Weichman \\ Condensed Matter Physics 114-36, California Institute of Technology, Pasadena, California 91125
}

(Received 24 June 1997)

\begin{abstract}
We study in light of recent ellipsometry, vapor pressure isotherm and specific-heat measurements on the thermodynamics of adsorbed thin films on graphite, the connection between the layering phase diagrams of thin films on periodic substrates and the thermodynamics of the solid-vapor interface of a semi-infinite crystal. The latter is the limit of the former when the film becomes infinitely thick, and we are interested in connecting this limiting behavior to the thermodynamics of films of finite thickness. We argue that the concepts of surface roughening, preroughening, and reconstruction provide a quantitatively useful framework within which to discuss this connection. Through general renormalization-group arguments and, in more detail, through a self-consistent mean-field treatment that explicitly accounts for all relevant phases, we show that the same types of interactions that lead to these different surface phases lead also to the reentrant layering transitions seen in the recent experiments. By appropriate tuning of the mean-field parameters we can semiquantitatively reconstruct all the observed experimental phase diagrams. It turns out that certain experimental phase diagrams with "zippers" require that the preroughening transition become first order. Our renormalization-group arguments predict such behavior in certain parameter ranges. In addition, for different parameters we predict the existence of an, as yet unobserved, $\theta$ disordered flat phase with spontaneously broken particle-hole symmetry and continuously varying surface height with an accompanying intermeshing layering phase diagram. The underlying lattice in the experiments is triangular, and this actually enhances the stability of the disordered flat phase and the corresponding reentrant layering transitions in the films. [S0163-1829(98)04307-0]
\end{abstract}

\section{INTRODUCTION}

\section{A. Surface critical phenomena}

The study of interfaces between two different thermodynamic phases has yielded a remarkable variety of interesting phenomena. Some of the most fascinating behavior occurs at the interface between a bulk semi-infinite crystal and its vapor. When the temperature is below the bulk triple point $T_{t}$ (the temperature at which the crystal melts in the presence of the vapor), the thermodynamics of the bulk crystal is smooth and nonsingular. The crystal surface, on the other hand, can exist in many different phases. The simplest phase is the flat phase in which the surface looks essentially like a bulk crystalline plane. This phase is characterized by the existence of a positive surface step free energy $f_{s}$, which discourages the formation of plateaus or depressions in the surface. Although a finite density of such imperfections will always be entropically favored, the probability of their occurence will decrease exponentially with their size. Furthermore, if the number of particles is such that the surface layer is incomplete, phase separation will occur and a single one-dimensional interface will separate two macroscopic flat regions with a unit height difference between them.

The flat phase is a special case of more general reconstructed phases. Here the surface layer, though only partially complete, nevertheless forms a periodic structure, commensurate with the underlying bulk crystal lattice plane, but with a larger unit cell, and a corresponding rational filling fraction $\theta_{R}$. There are analogous step free energies $f_{s, R}$, which discourage configurations of particles that deviate from perfect periodicity. If the number of particles is such that the overall filling fraction $\theta$ of the surface layer deviates from $\theta_{R}$, the surface will again phase separate with a single one- dimensional interface separating two (possibly different) reconstructed phases. In the event that the two phases are different, coexistence requires that the surface free energies must match.

Very different in character from the flat and reconstructed phases is the rough phase. At and above the roughening temperature $T_{r}<T_{t}$ the flat phase step free energy vanishes and it becomes entropically favorable for the surface to wander. To describe this quantitatively, let $\mathbf{r}=n_{1} \mathbf{a}+n_{2} \mathbf{b}$, where $n_{1}$ and $n_{2}$ are integers and $\mathbf{a}$ and $\mathbf{b}$ are primitive vectors, label the lattice points in the underlying crystal plane. Let $h(\mathbf{r})$ be the (integer) height of the surface above the lattice point $\mathbf{r}$. Then, at the roughening temperature, the variance of $h(\mathbf{r})$ diverges. More specifically, at and above $T_{r}$, the height-height correlation function,

$$
G\left(\mathbf{r}-\mathbf{r}^{\prime}\right) \equiv \frac{1}{2}\left\langle\left[h(\mathbf{r})-h\left(\mathbf{r}^{\prime}\right)\right]^{2}\right\rangle,
$$

increases logarithmically with separation:

$$
G(\mathbf{r}) \approx \frac{1}{4 \pi K_{R}(T)} \ln \left(r / a_{0}\right), \quad r \equiv|\mathbf{r}| \rightarrow \infty, \quad T_{r}<T<T_{t},
$$

where $a_{0}=|\mathbf{a}|$, say, is a microscopic length scale, and $K_{R}(T)$ may be thought of as a renormalized surface tilt modulus. In the flat and reconstructed phases, the variance $\langle[h(\mathbf{r})$ $\left.-\langle h(\mathbf{r})\rangle]^{2}\right\rangle$ is finite and equal to the large $r$ limit of $G(\mathbf{r})$.

The transition into the rough phase is in the universality class of the Kosterlitz-Thouless transition, which also describes the low-temperature magnetic ordering in the twodimensional $X Y$ model and the superfluid ordering in thin ${ }^{4} \mathrm{He}$ films. A consequence of this is that right at the roughening temperature, $T=T_{r}$, the renormalized tilt modulus has 
the universal value $K_{R}\left(T_{r}\right)=\pi / 2$. The value of $K_{R}$ jumps discontinuously to infinity below $T_{r}$, and decreases monotonically with $T$ above $T_{r}$. In the $X Y$ model the heights $h(\mathbf{r})$ appear in a dual representation of the original twocomponent spin model, and $1 / k_{B} T K_{R}(T)$ is proportional to the spin stiffness (or superfluid density) $Y$. There is an inverse relation $T \propto 1 / T_{X Y}$ between the temperatures in the two models since the flat phase, with $K_{R}(T) \equiv \infty$, corresponds to the disordered phase of the magnet (or superfluid), with $\mathrm{Y} \equiv 0 .{ }^{1}$

It turns out that there is yet another class of possible surface phases that may occur. These are the disordered flat (DOF) phases ${ }^{2-5}$ which may be thought of as intermediate between the reconstructed and rough phases. As an example, consider the (100) surface of a cubic crystal, and suppose that the atomic interactions are such that at low temperatures a kind of antiferromagnetic reconstructed phase with a checkerboard pattern $\left(\theta_{R}=\frac{1}{2}\right)$ is stabilized. Now, as the temperature rises, this phase may proceed directly through a roughening transition, analogous to that for the flat phase (but with a form of long-range antiferromagnetic order persisting). However, it is also possible, if the checkerboard pattern is only weakly stable, for the system to undergo an Ising transition that destroys long-range antiferromagnetic order without roughening the surface. The surface layer is then basically a two-dimensional lattice gas at half-filling. This phase is called the disordered flat phase. Raising the temperature further finally roughens the surface completely.

It is also possible to enter the DOF phase directly from the flat phase. ${ }^{2-5}$ The transition is driven by the entropy gain entailed by a disordered surface, and can occur even if the energetics favors the flat phase. Note that this transition causes a discontinuous change in the occupancy of the surface layer. If the total number of particles is fixed, this means that the surface must phase separate into two disordered flat phases, one with an extra half-layer of atoms, the other with a half-layer of "holes." The phase transition, at a temperature $T_{\mathrm{pr}}<T_{r}$, is called preroughening and lies in a different universality class from that of all the other transitions discussed so far. For example, the specific-heat exponent $\alpha$ can take any value between the Kosterlitz-Thouless value, $\alpha=$ $-\infty$, and the four-state Potts value, $\alpha=\frac{2}{3}$, depending upon the system parameters and, in particular, upon the precise strength of the tendency toward reconstruction ${ }^{4}$ (the more nearly stable the reconstructed phase, the larger the value of $\alpha)$. It turns out ${ }^{6}$ that the preroughening transition can even be driven first order, a possibility that was missed in earlier studies. $^{2-5}$

The disordering of the checkerboard phase is only one example of a DOF phase. In principle, corresponding to any reconstructed phase is a disordered flat phase with the same coverage $\theta_{R}$ separated from it by an Ising- (or perhaps Potts-) type phase transition. However, we shall see that DOF phases may also exist even without a corresponding reconstructed phase ever being stable. This is crucial for the triangular lattice substrates relevant to the experiments, where the analogue of the $\theta_{R}=\frac{1}{2}$ "antiferromagnetic" checkerboard reconstructed phase is frustrated and does not exist. Nevertheless, as we shall see, a $\theta=\frac{1}{2}$ disordered flat phase does exist, and is even more stable than its square lattice counterpart! In fact, there are conditions ${ }^{6}$ under which a disordered flat phase with continuously varying surface coverage $\theta(T)$ can exist. This $\theta \mathrm{DOF}$ phase was first proposed by den $\mathrm{Nijs}^{4}$ as a consequence of particle-hole symmetry breaking corner interactions. However, we find ${ }^{6}$ that the same physics that gives rise to the first-order preroughening mentioned above can, for different parameters, lead to a spontaneous breaking of particle-hole symmetry and corresponding $\theta$ DOF phase in a completely particle-hole symmetric model. Whether or not a given system will exhibit a disordered flat phase depends upon the detailed atomic interactions. It is clear that a rather sensitive balance of nearestand further-neighbor interactions may be required. ${ }^{2-5} \mathrm{~A}$ twocomponent "alloy" structure (as discussed in Sec. III and Appendix D) seems to be required to observe $\theta \mathrm{DOF}$ behavior.

\section{B. Layering critical phenomena}

Everything we have discussed so far relates to a free surface on a bulk semi-infinite crystal. This is important because it means that the potential experienced by an atom on the surface is an exactly periodic function of the number of layers: if a completed layer contains $N_{A}$ atoms, the addition to the surface of a further $N_{A}$ atoms yields a state thermodynamically indistinguishable from the original. It is this property that makes roughening and preroughening so different from more conventional two-dimensional critical phenomena.

If this discrete translational symmetry is broken, for example, by considering a crystalline slab of finite thickness, or by growing a finite number of layers of the crystal on a smooth substrate made of a different material, the surface critical phenomena will change. Thermodynamics will no longer be periodic in the number of layers, and the types of surface phases may change drastically from layer to layer. Historically, experimental work was directed mostly toward understanding monolayer physics. A rich variety of phenomena, including commensurate-incommensurate transitions between various registered and "floating" phases, ${ }^{7}$ reconstuction transitions, and dislocation mediated twodimensional melting, ${ }^{8}$ occur in very thin films. These phenomena have been explored experimentally using techniques such as heat capacity measurements, $x$-ray scattering, vapor pressure isotherms, neutron diffraction, and low-energy electron diffraction. ${ }^{9}$

However, our focus will be on multilayer phenomena. ${ }^{10}$ Thus we observe that, for a sufficiently large number of layers, the surface thermodynamics must, in some way, approach that of the perfect, bulk crystal surface. Conversely, the bulk surface phases and phase transitions must be reflected somehow in the behavior of a finite but sufficiently thick film. Motivated by the results of some recent experiments on rare gases adsorbed on graphite $\mathrm{e}^{11-14}$ and $\mathrm{MgO}$ (Ref. 15) substrates, the purpose of this paper is to explore precisely this latter issue. Figure 1 constitutes a complete pictorial summary of our results. All phase diagrams in this figure have been computed using a sophisticated plaquette mean-field theory, to be introduced in later sections, applied to the restricted solid-on-solid (RSOS) model. The model, which will be introduced in detail in Sec. II, contains two parameters, $K=J_{1} / k_{B} T$ and $L=J_{2} / k_{B} T$, where $J_{1}$ and $J_{2}$ 

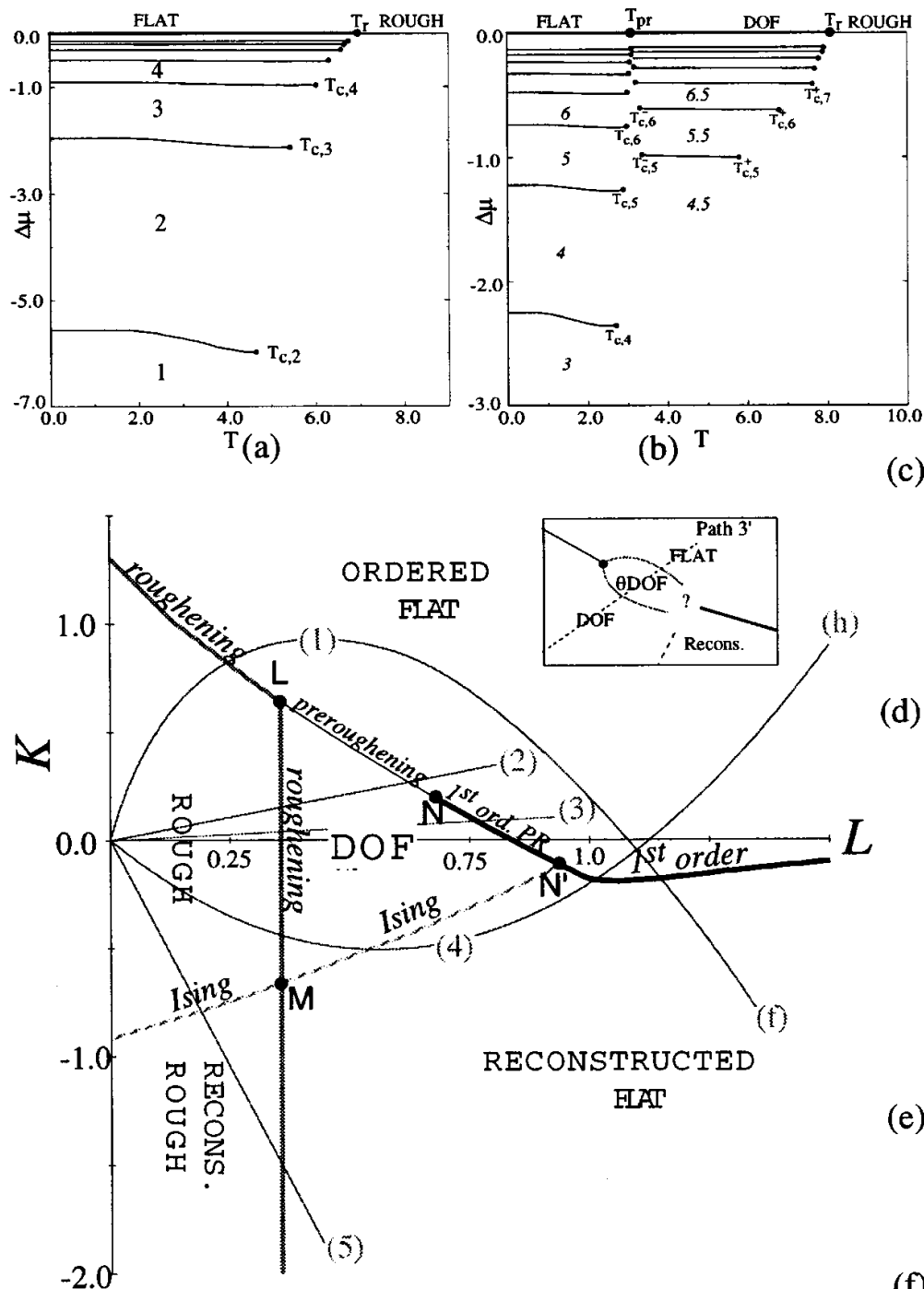

(h)
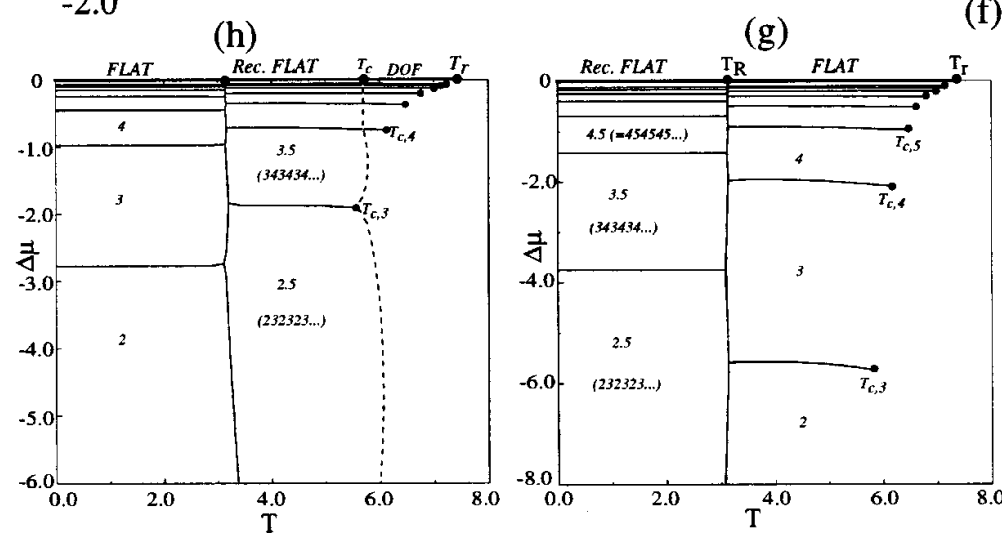

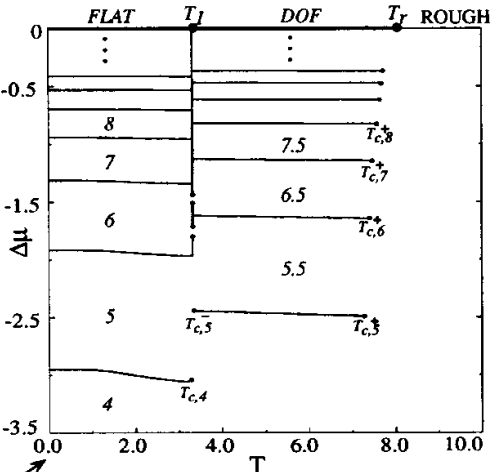

(c)

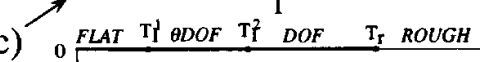

FIG. 1. Pictorial summary of the essential results in this paper. The central plot shows a global phase diagram, as computed using a plaquette mean-field theory on a square lattice, for the RSOS model of a bulk interface, with $K=J_{1} / k_{B} T$ and $L=J_{2} / k_{B} T$ the nearest- and next-nearest-neighbor interactions, showing the six possible different surface phases. Strictly speaking, all the roughening lines are actually absent in mean-field theory. The theory instead shows a narrow crossover that we have used to estimate the positions of these lines (see the discussion in Sec. IV C). All other transitions, however, are sharp. The inset shows schematically an alternative scenario containing the $\theta$ DOF phase. This scenario is not found in the RSOS model we study (hence the remaining questions about how some of the transition lines connect up), but is expected to appear in other models. Paths 1-5 represent possible experimental trajectories through this phase diagram. The surrounding figures show the layering phase diagrams associated with these paths when a substrate potential is included: (a) Pure roughening behavior and associated low-temperature layering transitions, path 1. (b) Continuous preroughening behavior and associated reentrant layering, path 2. (c) First-order preroughening behavior and associated zippering, path 3. (d) $\theta$ DOF phase behavior and associated intermeshing, path 3 ' [inset to the central plot]. The layering phase diagram in this case is computed from the sine-Gordon theory (Sec. III) rather than the mean-field theory since our RSOS model does not show this behavior. (e) DOF to reconstructed behavior and associated antiferromagnetic transitions within each layer, path 4. (f) Reconstructed-rough behavior and associated surrounding antiferromagnetic line, path 5. (g) First-order flat to reconstructed behavior, similar to (b) but with layering lines reversed, extension of path 1. (h) First-order reconstructed to flat behavior, similar to (c) but with an antiferromagnetic line at higher temperature, extension of path 4. A similar extension of path 5 (not shown) would move this antiferromagnetic line outwards, as in (g), to surround the layering lines. 
are, respectively, nearest- and second-nearest neighbor interactions between the surface heights $h(\mathbf{r})$. For present purposes one need only know that positive $J_{1}$ energetically favors neighboring columns of equal height, while negative $J_{1}$ favors a unit height difference; $J_{2}$ is always kept positive, and favors second neighboring columns of equal height. The central plot in Fig. 1 is the bulk interface phase diagram for this model. We see clearly here the six different phases we have discussed, and the transition lines between them. Shown also are five different experimental paths through the phase diagram that we will outline here, and consider in detail in later sections. Associated with each of these paths is a layering phase diagram, Figs. 1(a)-(h), corresponding to the same RSOS model but now including a substrate potential.

Path 1 shows ordinary surface roughening behavior. The relation between this behavior and layering critical phenomena is actually well known (see especially Ref. 16): the roughening temperature $T_{r}$ is the accumulation point for the sequence of critical points $T_{c, n}$ that terminate the first-order layering transitions at lower temperatures [see Fig. 1(a)]. The $n$th layering line separates phases with approximately integer film thicknesses, $n-1$ and $n$, and ends in an Ising critical point $T_{c, n}$.

Paths 2, 3, and 3' cut, in various ways, through the $J_{1}$ $>0$ portion of the DOF phase, and are the primary focus of this paper. Path 2 corresponds to ordinary preroughening. Den Nijs has proposed some possible associated layering phase diagrams. ${ }^{5}$ The basic idea is that there should be two sequences of layering transitions. At low temperatures $T$ $\leq T_{\mathrm{pr}}$ there is a sequence of first-order layering transitions between integer coverages, while at higher temperatures $T_{\mathrm{pr}}$ $\leq T \leq T_{r}$ there is a sequence of first-order layering transitions between integer-plus-one-half (or, more generally, integerplus- $\theta_{R}$ ) coverages. The second set of lines must therefore be reentrant, with upper and lower endpoints $T_{c, n}$ and $T_{n}^{2}$, respectively. The low-temperature set have only upper endpoints $T_{n}^{1}$. The temperatures $T_{c, n}$ still tend to $T_{r}$ as $n \rightarrow \infty$. What was not previously understood is in what way (if at all) the endpoints $T_{n}^{2}$ and $T_{n}^{1}$ are connected together. Den Nijs ${ }^{5}$ suggests two possibilities: (i) $T_{n}^{1}$ and $T_{n}^{2}$ are Ising critical points, as are $T_{c, n}$, with $T_{n}^{1}, T_{n}^{2} \rightarrow T_{\mathrm{pr}}$, and are not connected in any way [Fig. 1(b)]; (ii) $T_{n}^{2}$ and $T_{n}^{1}$ are triple points, zipped together by a zigzagging sequence of first-order lines, with $T_{n}^{1}, T_{n}^{2} \rightarrow T_{0}$ [Fig. 1(c)]. We distinguish between $T_{\mathrm{pr}}$ and $T_{0}$ for reasons that will become obvious below. Another possibility (iii) is that the two sets of layering lines intermesh, with distinct limits $T_{n}^{1} \rightarrow T_{I}^{1}$ and $T_{n}^{2} \rightarrow T_{I}^{2}$ where $T_{I}^{2}<T_{I}^{1}$ [Fig. 1(d)]. The high-resolution heat capacity studies ${ }^{12-14}$ suggest possibility (ii). We will show that, depending on the parameters, all of these possibilities, as well as others, can occur. Possibility (i) indeed corresponds to a continuous preroughening transition, path 2; while (ii) corresponds to a firstorder transition between flat and DOF phases ( $T_{0}$ denoting then the first-order preroughening temperature), path 3; and (iii) to a $\theta$ DOF phase in the temperature interval $T_{I}^{2} \leqslant T$ $\leqslant T_{I}^{1}$, path $3^{\prime}$. The bulk interface transitions $T_{I}^{2}$ and $T_{I}^{1}$ are also Ising like, and in the $\theta \mathrm{DOF}$ phase one has a continuously varying surface coverage, $0 \leqslant \theta(T) \leqslant \frac{1}{2}$ with $\theta\left(T_{I}^{1}\right)=0$ and $\theta\left(T_{I}^{2}\right)=\frac{1}{2}$.
Although not relevant to present experiments, one may also cut through the DOF phase with $J_{1}<0$, ending with a reconstructed phase at lower temperatures. This is represented by path 4 , and the associated layering phase diagram is shown in Fig. 1(e). Here there is only one set of layering lines, between half-integer coverages. However, for each given film thickness an Ising antiferromagnetic ordering transition takes place at intermediate temperatures. This transition becomes the DOF-reconstructed phase boundary on the bulk interface. The roughening transition at higher temperatures is again reflected in the sequence of Ising critical points $T_{c, n}$.

Path 5 shows behavior for larger $\left|J_{1}\right| / J_{2}$, where the surface roughens before it deconstructs, yielding an intermediate reconstructed-rough phase. The associated layering phase diagram [Fig. 1(f)] displays a sequence of layering transitions between half-integer film thicknesses lying completely within an antiferromagnetic phase boundary. The nature of the order within this boundary is quite subtle, corresponding to antiferromagnetic order in the magnitude of the mean-square fluctuations of each column height, not in the column heights themselves. The latter symmetry is broken only below $T_{c, n}$.

We also show layering phase diagrams for somewhat fanciful continuations of paths 1 and 4 that cross the first-order flat to reconstructed phase boundary [see Figs. 1(g,h)]. There is no experimental evidence for $J_{1}$ changing sign as a function of $T$, but the resulting phase diagrams are remarkably similar in appearance to Fig. 1(c), associated with first-order preroughening, and the experimental results for Argon and Krypton on graphite. ${ }^{12,13,17}$ The continuation of path 1, shown in Fig. 1(g), is identical to Fig. 1(c), except that the integer and half-integer layering lines are interchanged. Ellipsometry ${ }^{11}$ and vapor pressure isotherm measurements, ${ }^{12-14,18}$ however, are sufficiently accurate to rule out such an interchange. The continuation of path 4 shown in Fig. 1(h) has the two sets of layering lines in the correct order, but, just as in Fig. 1(e), involves also an antiferromagnetic Ising phase boundary. A similar extension of path 5 (not shown) would detach this Ising boundary completely from the layering lines, exactly as in Fig. $1(\mathrm{~g})$, and the physics would then have nothing to do with the DOF phase at all. We have not shown this latter phase diagram explicitly because the mean-field theory becomes numerically very hard to control for larger $\left|J_{1}\right| / J_{2}$ (see the discussion in Sec. II C 2). Once again, there is no evidence for any of these scenarios in any of the experiments.

As mentioned, the experimental graphite substrate lattice is triangular. Indications are that the reentrant layerings nevertheless occur at half-filling. Although $\theta_{R}=\frac{1}{2}$ reconstructed phases, such as those with every second row missing, do exist on a triangular lattice, they do not arise in a natural way if the interactions are isotropic. It is likely, then, that for the models we consider here there is no stable half-filled reconstructed phase. Until now, this was thought to be a problem for the DOF phase interpretation of reentrant layering. ${ }^{5}$ What we will show, however, is that the absence of a reconstructed phase actually enhances the DOF phase, and that there are two factors that one must consider in determining the filling fraction $\theta$ at which it occurs. Thus, although it is energetics that favors a DOF phase with filling fraction $\theta_{R}$, it is entropy 


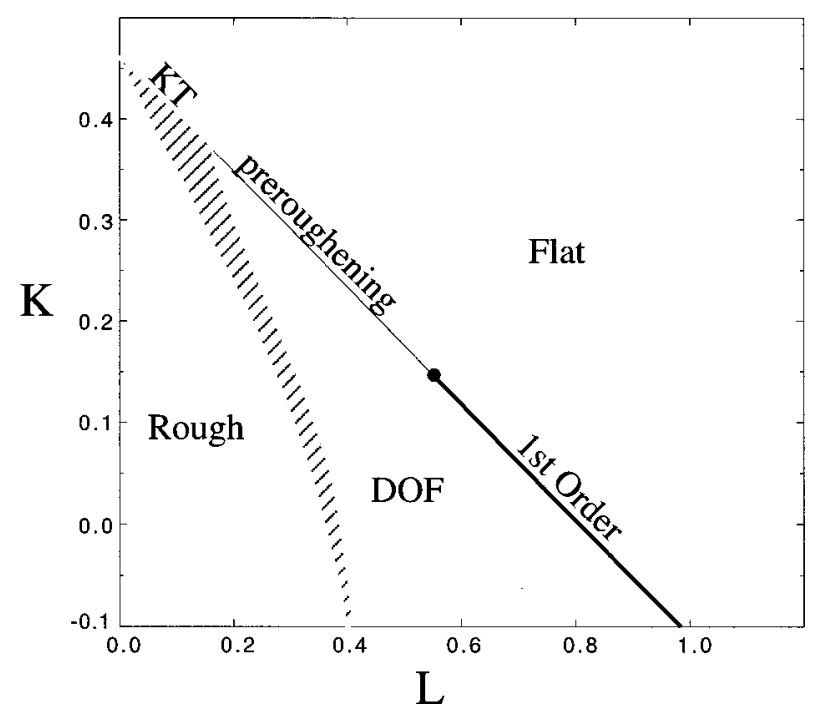

FIG. 2. Global phase diagram, as computed using a plaquette mean-field theory on a triangular lattice, for the RSOS model of a bulk interface. The reconstructed and reconstructed rough phases, and transitions associated with them, are now absent, leading to a much enlarged DOF phase. The behavior for $K>0$, however, is qualitatively unchanged from that for a square lattice, Fig. 1. In particular, paths 1,2 , and 3 exhibit behavior qualitatively identical to that shown in Figs. 1(a)-(c). Path 4 yields behavior similar to that in Fig. 1(e) except that the antiferromagnetic line is now absent - the upper layer remains disordered to arbitrarily low $T$. The roughening line is again estimated (see the discussion in Sec. IV C).

that drives the preroughening transition and disfavors integer filling fractions. In the absence of the former, the latter will tend to form a DOF phase halfway between the two bounding integer coverages even in the absence of an "attracting", incipient reconstructed phase, just as seen in the experiments. The triangular lattice bulk interface phase diagram, as computed using our plaquette mean-field theory, is shown in Fig. 2. As can be seen, the main difference between this figure and the central phase diagram in Fig. 1 is the absence of the reconstructed and reconstructed rough phases, and the correspondingly expanded DOF phase. The $K>0$ portion of the phase diagram is, however, qualitatively unchanged.

\section{Outline}

In this paper we will examine various solid-on-solid models of surface critical phenomena in the presence of a substrate potential. It is our aim to understand the conditions under which possibilities (i), (ii), (iii) (or perhaps something entirely different) occur. Much can be understood qualitatively based on the sine-Gordon (closely related to the Coulomb gas) representation of the roughening and preroughening transitions, ${ }^{4}$ generalized to include a substrate potential. However our main quantitative tool will be a mean-field theory sophisticated enough to account for all of the possible surface phases. Since the issue here is really the topology of the phase diagram, rather than the nature of the critical points (which are all Ising like for finite $n$, and, in any case, the experiments do not resolve detailed critical behavior) one can go a long way with mean-field theory, even to the point of obtaining semiquantitative results.
In Sec. II we introduce the RSOS models of crystal-vapor interfaces and discuss their general properties. A great deal of intuition can be obtained by considering the limit of a strong substrate potential and restricting the model to a small number (two or three) of layers. One then obtains effective spin- $j$ (with $j=\frac{1}{2}$ or $j=1$ ) Ising models whose phase diagrams can be understood quite generally. A plaquette meanfield formalism is then developed for later detailed computations.

In Sec. III we will use generalized sine-Gordon models along with renormalization-group arguments to discuss the phenomenology of the layering phase diagram. In so doing we will uncover the four basic classes of layering behavior for thick films shown in Figs. 1(a)-(d).

In Sec. IV we explore solutions to the mean-field equations, classifying, to some extent, the possible phase diagrams. We find that the RSOS model exhibits, depending on parameters, the first three behaviors described above, but not the fourth. As mentioned, the $\theta \mathrm{DOF}$ phase requires a more general "alloy" RSOS model. In addition, we explore a number of phase diagrams that do not have sine-Gordon model descriptions, namely, those that involve reconstruction. As alluded to above, some of these mimic closely some of the phase diagrams involving preroughening, but there are significant experimentally observable differences.

In Sec. V we conclude by comparing the theoretical and experimental phase diagrams. We also describe future work that might help in the search for new experimental systems that display the so far unobserved phase diagrams.

Various appendices contain more technical derivations. Appendix A contains a formal development of consistent plaquette mean-field theories. In Appendixes B and C explicit expressions for the free energies on various lattices and for various plaquettes are derived. In Appendix D the equivalence between the body-centered-cubic solid-on-solid (BCSOS) model and the Ashkin-Teller model is outlined and this is used to exhibit an RSOS "binary alloy" model with a $\theta$ DOF phase.

\section{MODELS AND METHODS}

\section{A. Solid-on-solid models}

Solid-on-solid (SOS) models are conventionally used to model interface phenomena. In these models the vapor phase above the surface is taken to be a perfect vacuum, while the solid phase below is taken to be a perfect crystal, and surface overhangs are ignored. The surface is then defined by a set of column heights $h(\mathbf{r})$ above a two-dimensional lattice spanned by the index $\mathbf{r}$. To begin with we shall assume a simple square lattice with $h(\mathbf{r})$ taking integer values. Later on we shall discuss the experimentally more relevant case of a triangular lattice. In fact, the bulk crystals considered here have a face-centered-cubic structure in which sequential layers of atoms sit in the interstices of the previous layer. Although, for a given $\mathbf{r}, h(\mathbf{r})$ can change only in integer steps, neighboring heights will then differ by noninteger amounts. For simplicity of modeling, we shall ignore this complication and take the triangular lattices to lie one on top of the other so that all $h(\mathbf{r})$ are integers. ${ }^{19}$ In the restricted solid-on-solid models, the further constraint is imposed that neighboring column heights can differ by at most unity. This reflects the physical constraint that it is energetically unfavorable to 


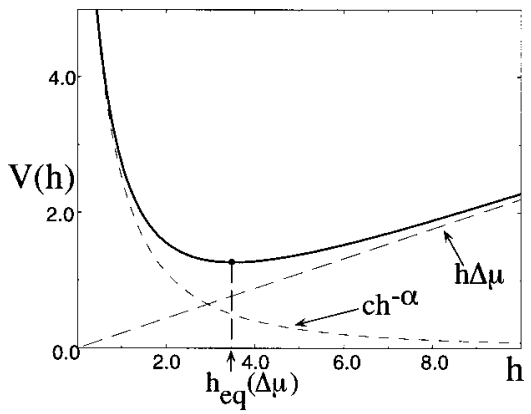

FIG. 3. Substrate potential, $V(h)$.

form steps of greater than unit height. In the RSOS models the energy barrier against such steps is simply taken to be infinite. This constraint greatly reduces the number of surface configurations and, therefore, simplifies certain analytic and numerical calculations (see below) without affecting the basic physics. It also decreases the configurational entropy, and roughness, of the surface relative to that of, say, the interface between oppositely magnetized domains in a threedimensional Ising model. This tends to stabilize more delicate phases, like the disordered flat phase, which rely on a critical balance between configurational entropy and step free-energy barriers. Clearly, whether the SOS model, RSOS model, or something in between, is most appropriate depends upon the details of the system being modeled.

Following den Nijs, ${ }^{4}$ we first consider the RSOS Hamiltonian on a square lattice,

$$
\begin{aligned}
\mathcal{H}= & \frac{1}{2} J_{1} \sum_{\left\langle\mathbf{r}, \mathbf{r}^{\prime}\right\rangle}\left[h(\mathbf{r})-h\left(\mathbf{r}^{\prime}\right)\right]^{2}+\frac{1}{2} J_{2} \sum_{\left(\mathbf{r}, \mathbf{r}^{\prime \prime}\right)}\left[h(\mathbf{r})-h\left(\mathbf{r}^{\prime \prime}\right)\right]^{2} \\
& +\sum_{\mathbf{r}} V[h(\mathbf{r})],
\end{aligned}
$$

where the first sum is over nearest neighbors and the second sum is over second (i.e., diagonal) neighbors. We assume $J_{2}>0$ always, but $J_{1}$ can be either positive or negative. The external potential, $V(h)$ [in the absence of which, Eq. (2.1) is precisely the model treated in Ref. 4] is due to the substrate, and takes the form ${ }^{16}$ (see Fig. 3)

$$
V(h)=\left\{\begin{array}{l}
h \Delta \mu+v(h), \quad h \geqslant 0 \\
\infty, \quad h<0,
\end{array}\right.
$$

with $v(h) \approx c h^{-\alpha}$ for large $h .^{20}$ For a van der Waals substrate potential, $\alpha=2$ and $c>0$. The linear coefficient, $\Delta \mu$ $=\mu_{\text {coex }}-\mu$, is the deviation of the chemical potential from bulk solid-vapor coexistence. For $\Delta \mu>0$ the bulk phase is vapor, while for $\Delta \mu<0$ the bulk phase is solid. A true bulk equilibrium interface exists only for $\Delta \mu=0$ (precisely analogous to external magnetic field $H=0$ in an Ising model). If $J_{1}$ and $J_{2}$ are both positive, then at zero temperature the interface is perfectly flat and its equilibrium position is at the minimum, $h_{0}(\Delta \mu, T=0)$, of $V(h)$ (over integer values of $h$ ). For small $\Delta \mu$ the minimum diverges as

$$
h_{0}(\Delta \mu) \approx\left(\frac{\alpha c}{\Delta \mu}\right)^{1 /(1+\alpha)} \sim \Delta \mu^{-1 / 3}, \quad \Delta \mu \rightarrow 0 .
$$

It is for this reason that absorption isotherms, which essentially measure $h_{\mathrm{eq}}(\Delta \mu, T) \equiv\langle h(\mathbf{r})\rangle$ as a function of $\Delta \mu$ for fixed $T$, are often plotted versus $\Delta \mu^{-1 / 3}$ : the steps due to the sequence of layer completions then occur with roughly equal spacing. ${ }^{12,13}$

The physics behind Eq. (2.1) is as follows. Consider first a bulk interface with $V \equiv 0$. If $J_{1}$ is positive and large compared to $J_{2}$, the energetics give preference to a flat interface, and the model will produce a standard roughening transition with increasing temperature when $K \equiv J_{1} / k_{B} T$ and $L$ $\equiv J_{2} / k_{B} T$ are sufficiently small. If $J_{1}<0$ and is large in magnitude compared to $J_{2}$, neighboring column heights prefer to differ by unity. However, since $J_{2}$ prefers that diagonal nearest-neighbor column heights have equal height, an antiferromagnetic order is stabilized at low temperature: this is the checkerboard reconstructed phase. As $K$ decreases, this phase roughens, but still retains a generalized long-range antiferromagnetic order. $^{2}$ A second Ising-like transition, at higher temperature, into a fully rough phase is required to finally eliminate this residual order. However, if $J_{1}<0$ is sufficiently small in magnitude, the antiferromagnetic order can be lost, via an Ising transition, before the surface roughens: this is the transition to the disordered flat phase. This phase actually persists also for small $J_{1}>0$ : the entropy gain from disordering the surface more than offsets the loss of ferromagnetic energy. The central plot in Fig. 1 shows how these four phases fit together.

Now, how are these phases affected by the presence of $V(h)$ ? The effect on the rough phases is catastrophic! Since $V(h)$ prefers a set of values of $h$ near $h_{0}(\Delta \mu)$, the correlation function $G(\mathbf{r})$ [see Eq. (1.1)] must always remain finite as $|\mathbf{r}| \rightarrow \infty$. The logarithmic divergence in Eq. (1.2) must saturate. We may estimate the saturation value as follows: assuming that the interface does not wander too far from the minimum, it will be governed by the effective Hamiltonian,

$$
\overline{\mathcal{H}}_{\text {eff }} \equiv \frac{\mathcal{H}_{\mathrm{eff}}}{k_{B} T}=\frac{1}{2} \int d^{2} r\left[K_{R}|\nabla h|^{2}+\kappa\left(h-h_{0}\right)^{2}\right],
$$

where

$$
k_{B} T \kappa=\left(\frac{\partial^{2} V}{\partial h^{2}}\right)_{h=h_{0}} \approx \alpha(\alpha+1)\left(\frac{\Delta \mu}{\alpha c}\right)^{(2+\alpha) /(1+\alpha)} \sim \Delta \mu^{4 / 3}
$$

is the curvature at the minimum, and $K_{R}$ is the effective long-wavelength (renormalized) tilt modulus [see Eq. (1.1)] in the absence of $V$. This Hamiltonian is Gaussian, and yields

$$
\begin{aligned}
\left\langle\left(h-h_{0}\right)^{2}\right\rangle & \approx \int_{q \leqslant \pi / a} \frac{d^{2} q}{(2 \pi)^{2}} \frac{1}{K_{R} q^{2}+\kappa}=\frac{1}{4 \pi K_{R}} \ln \left[1+\frac{\pi^{2} K_{R}}{\kappa a^{2}}\right] \\
& \approx \frac{2+\alpha}{1+\alpha} \frac{1}{4 \pi K_{R}} \ln \left(\frac{c}{\Delta \mu}\right) \ll \frac{1}{\kappa}
\end{aligned}
$$

which also estimates the saturation value of $G(\mathbf{r})$. The final inequality tells us, self-consistently, that although the interface width diverges logarithmically as $\Delta \mu \rightarrow 0$, the interface remains sufficiently close to $h_{0}$ that the quadratic approximation remains valid. 
Clearly, the flat phases will be affected by the potential in much more subtle ways. They will, of course, remain flat. The question we address is the nature of the various transitions between them in the presence of $V(h)$.

\section{B. Effective layer Hamiltonians}

From the general Hamiltonian (2.1) one can derive various approximate effective Hamiltionians for describing the thermodynamics of individual layers. The basic idea is that if the effective potential $V(h)$ increases rapidly to either side of the minimum near $h_{0}(\Delta \mu)$, then large deviations of the column heights from $h_{0}$ will be strongly discouraged, and, to a good approximation, one can suppress all values of $h(\mathbf{r})$ outside of some narrow range. If this range encompasses an integer $2 j+1$ of values, one then has reduced the full Hamiltonian to one of a classical spin- $j$ Ising model. It will transpire that a description of the thin-film analogue of the disordered flat phase requires $j \geqslant 1$. However, we will begin our discussion with the simpler spin $-\frac{1}{2}$ model.

\section{Spin-j Ising models}

A spin- $\frac{1}{2}$ description is valid if the the substrate potential is so strong as to allow essentially only one value of the column heights, except when the value of $\Delta \mu$ is such that two column heights, say $n$ and $n+1$, are nearly degenerate in energy. In this latter situation the true minimum of $V(h)$ lies near $n+\frac{1}{2}$, and $V(n) \approx V(n+1)$. Physically, we expect this to be a valid description for films only a few layers thick. We define the spin- $\frac{1}{2}$ variables $s(\mathbf{r})$ via

$$
s(\mathbf{r})= \begin{cases}-1 & \text { if } h(\mathbf{r})=n \\ +1 & \text { if } h(\mathbf{r})=n+1\end{cases}
$$

Ignoring all other possible values of $h(\mathbf{r})$, the Hamiltonian now becomes

$$
\begin{aligned}
\overline{\mathcal{H}} \approx \overline{\mathcal{H}}_{1 / 2} \equiv & \frac{1}{2} K \sum_{\left\langle\mathbf{r r}^{\prime}\right\rangle}\left[s(\mathbf{r})-s\left(\mathbf{r}^{\prime}\right)\right]^{2}+\frac{1}{2} L \sum_{\left(\mathbf{r r}^{\prime \prime}\right)}\left[s(\mathbf{r})-s\left(\mathbf{r}^{\prime \prime}\right)\right]^{2} \\
& -h \sum_{\mathbf{r}} s(\mathbf{r})
\end{aligned}
$$

where $\overline{\mathcal{H}} \equiv \mathcal{H} / k_{B} T, \quad \overline{\mathcal{H}}_{1 / 2} \equiv \mathcal{H}_{1 / 2} / k_{B} T, h=H / k_{B} T$ with $H$ $=\frac{1}{2}[V(n)-V(n+1)]$ an effective magnetic field, and we have dropped an overall constant term, $C=\frac{1}{2}[V(n)+V(n$ $+1)] N_{A}$ where $N_{A}$ is the number of atoms per layer. We should really distinguish between the coupling constants $K$ and $L$ that appear in Eq. (2.8) and those that appear in Eq. (2.1) because the former are effective parameters that will differ somewhat from the latter in a way that depends upon how good an approximation the spin- $\frac{1}{2}$ model is. For simplicity of notation, however, we will not make this distinction explicit. For $L=0$ this is the standard two-dimensional Ising Hamiltonian. If $K>0$ the model is ferromagnetic, and when $H=0$ there is a phase transition to a state with finite magnetization as $K$ increases through a critical value $K=K_{c}$ [see Fig. 4(a)]. If $K<0$ the model is antiferromagnetic. Since $H$ does not couple directly to the staggered magnetization order parameter in this case, there is a line of transitions, $K$ $=K_{c}(H)$ [see Fig. 4(b)], to states with finite staggered magnetization. Thus, although $H$ polarizes the spins somewhat, (a)

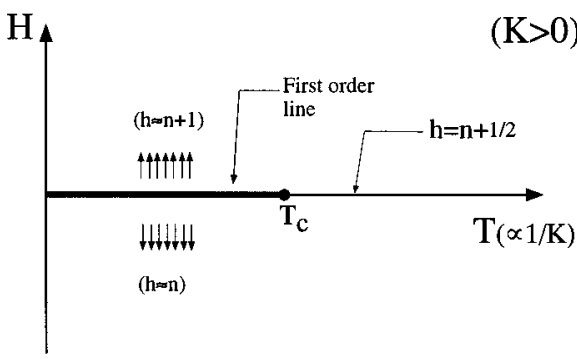

$(\mathrm{K}<0)$

(b)

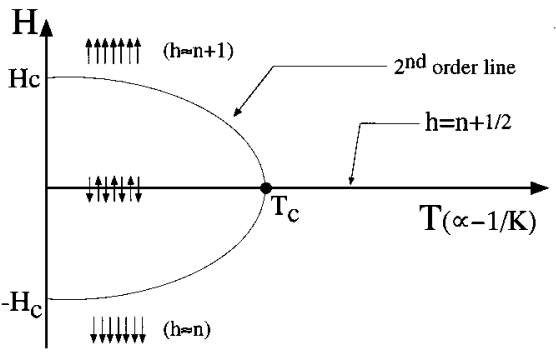

FIG. 4. (a) Ferromagnetic and (b) antiferromagnetic Ising phase diagrams for $L=0$.

antiferromagnetic order survives if $H$ is not too large. Clearly, one must have $K_{c}(0)=-K_{c}$. This line terminates at $T=0(K=-\infty)$ for a critical value of the field, $H= \pm H_{c}$, with $H_{c}=-2 J_{1}$. Since $L>0$ encourages the alignment of diagonal nearest-neighbor spins, it enhances both ferromagnetic and antiferromagnetic order. If $L$ is not too large, the phase diagrams are qualitatively unchanged.

For large $L>0$, new behavior occurs. Suppose $K=0$. Then the two interpenetrating sublattices are decoupled, and $L$ provides a nearest-neighbor ferromagnetic coupling within each one. Thus, at $H=0$ and a critical value, $L=K_{c}$, the two sublattices will independently order ferromagnetically. We may view a small value of $K$ as a perturbation on this behavior, which then determines how these two sublattices orient relative to one another. If $K>0$ (but arbitrarily small) the two will order parallel to each other, yielding an overall ferromagnetic state; if $K<0$ (but arbitrarily small) the two will order antiparallel to each other, yielding an overall antiferromagnetic state. There is, therefore, a first-order transition from one ordered state to the other when $K$ reverses sign at large enough $L$. This is seen in the $K-L$ plane at $H=0$ in Fig. $5(\mathrm{~b})$.

For nonzero $H$ the ferromagnetic part of the critical line is destroyed [see Fig. 5(b)], but the antiferromagnetic part survives, and must merge somehow with the extension of the first-order decoupling line, $K=0, L>K_{c}$. For large $L$ it is easy to see that the latter moves to negative $J_{1} \simeq-\frac{1}{2}|H|$ since a finite $K<0$ is now required to overturn one sublattice against the field. For small $L$ the transition remains secondorder. How the two behaviors connect at intermediate $L$ is surprisingly complicated: for smaller $H$ the two meet in a tricritical point, while for larger $H$ the second order line ends in a critical endpoint on the first-order line, while the firstorder line ends in an Ising critical point inside the antiferro- 
(a)
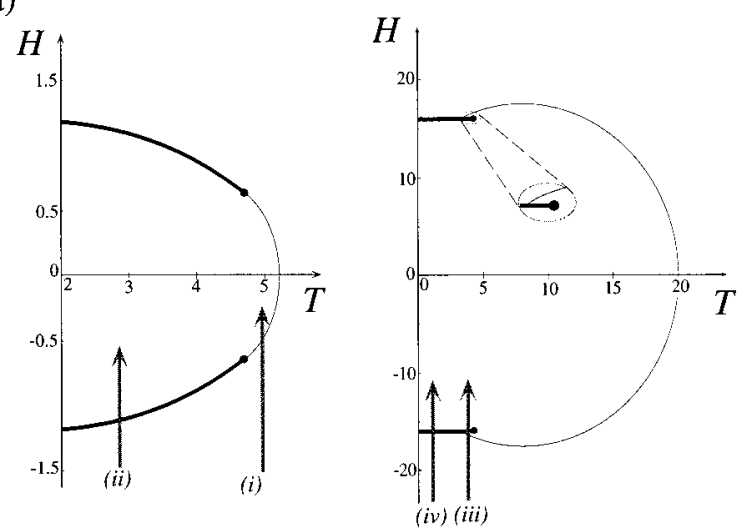

(b)

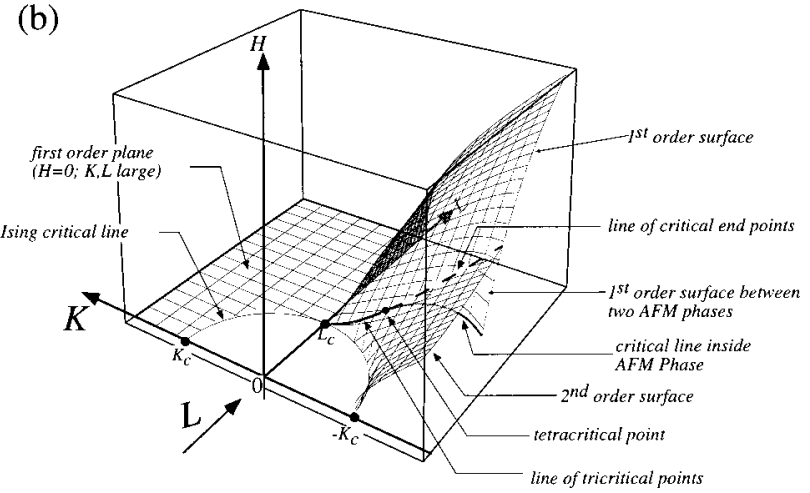

FIG. 5. Ising phase diagrams for $L>0$ : (a) $H$ vs $T$ phase diagrams showing tricritical behavior for $J_{2} /\left|J_{1}\right|>j_{4 c}$ and critical-endpoint behavior for $J_{2} /\left|J_{1}\right|<j_{4 c}$. Figure $4(\mathrm{~b})$ is recovered as $J_{2} /\left|J_{1}\right| \rightarrow 0$. The paths labeled (i), (ii), (iii), and (iv) refer to the corresponding parts of Fig. 19 below. (b) Three-dimensional plot, with details of the tricritcial and critical-end-point structure shown.

magnetic phase. A tetracritical point, at a particular value of $H=H_{c 4}$, separates these two behaviors. This is shown schematically in Fig. 5(a). A three-dimensional phase diagram in the full $H-K-L$ space is shown in Fig. 5(b). All this will be described in more detail in Sec. IV.

Let us now understand the relationship between this phase diagram and the layering transitions in the solid-on-solid model. Consider first $K>0$ and $L=0$. At low temperature $K$ will be larger than $K_{c}$, and as $H$ passes through zero a firstorder transition will take place between the spin-down ferromagnetic phase and the spin-up ferromagnetic phase. This corresponds to a first-order layering transition (as a function of $\Delta \mu$ ) between $n$ completed layers, with a dilute gas of atoms (whose density varies continuously with $H<0$ ) in the partially completed $(n+1)$ st layer, and $n+1$ completed layers with a dilute gas of "holes" (whose density varies continuously with $H>0)$ in the $(n+1)$ st layer, occurring precisely when $V(n)$ and $V(n+1)$ are degenerate. This firstorder line terminates in an Ising critical point, above which the layers grow continuously.

As $\Delta \mu$ decreases further, $V(n+2)$ eventually becomes degenerate with $V(n+1)$, and we leave the domain of validity of the Ising model (2.8). However, we may now ignore the $n$th layer, which is essentially full and inert, and consider a new effective Ising model, of the same form as Eq. (2.8), for the $(n+1)$ st and $(n+2)$ nd layers. The effective param- eters will be slightly different since the precise shape of $V(h)$ has changed, but the same physics will now repeat, with $H=\frac{1}{2}[V(n+1)-V(n+2)]$. In particular, a new layering transition between $n+1$ and $n+2$ layers will now occur. Repeating this whole process indefinitely generates the entire infinite sequence of layering transitions [Fig. 1(a)]. Of course, our assumption that $V(h)$ effectively isolates only two layers breaks down as the number of layers increases, but the picture actually remains valid. The point is that $K$ prefers a flat surface, and Huse ${ }^{16}$ has shown that the endpoints of the layering transitions accumulate at the roughening transition from the low-temperature side. Therefore the renormalized tilt modulus $K_{R}$ in Eq. (2.4) is still infinite, and the interface is flat right through the Ising transition. It is therefore a combination of a weak minimum in $V(h)$ and the fact that $T_{c, n}<T_{r}$ that maintains the correctness of our simple picture. These results are qualitatively unaffected if $L>0$ since $L$ just enhances the stability of the flat phase somewhat.

Consider next $K<0$. The original RSOS Hamiltonian does not really make sense in this case if $J_{2}=0$ since the surface will always be rough: in the absence of a strongly localizing substrate potential, one needs a finite $J_{2}$ to stabilize a flat surface at low temperatures. The corresponding effective layer Hamiltonian must then have a positive $L$. At low temperatures, then, the first-order layering lines now broaden out into second-order lobes enclosing checkerboard ordered phases that exist in the interval $-H_{c}(T)<H$ $<H_{c}(T)$ [see Figs. 4(b) and 5(a)]. As above, there will be one such lobe for each value of $n$. If $V(h)$ is sufficiently steep so that $H$ passes through $H_{c}$ before $V(n+2)-V(n$ $+1)$ becomes smaller than $8\left|J_{1}\right|$, then the transition line reaches right to $T=0$ and is completely disjoined from the checkerboard phases at neighboring coverages. In principle, all of the complicated triple-point or critical-end-point structure will appear as well. This is shown towards the bottom of Fig. 1(e). If, on the other hand, $V(n+2)-V(n+1)$ becomes smaller than $8\left|J_{1}\right|$ before $H$ passes through $H_{c}$, the neighboring lobes will overlap and one will have a first-order transition between neighboring checkerboard phases at low temperature. This must happen for sufficiently large $n$, and is shown in the upper-left-hand parts of Fig. 1(e).

What happens at higher temperatures? There are two possibilities, depending upon the relative strengths of $J_{1}$ and $J_{2}$. If $J_{2}$ is large compared to $J_{1}$, then the layering tendency is stronger than the reconstruction tendency, and will survive to higher temperatures. Therefore, as the temperature rises, first the reconstructed phase disorders, while the strong $L$ continues to maintain a flat, roughly half-filled surface. The second-order antiferromagnetic Ising transitions then terminate at critical endpoints on the first-order layering lines. As the film thickens the reconstruction transitions accumulate at the bulk surface reconstruction transition, $T=T_{R}$. Meanwhile, the first-order layering lines terminate at Ising critical points $T_{c, n}$ at higher temperatures. These critical points acculmulate at the bulk surface roughening transition, $T=T_{r}$. The bulk surface phase in the interval $T_{R}<T<T_{r}$ is precisely the disordered flat phase. This scenario is pictured in Fig. 1(e).

If, on the other hand, $J_{1}$ is large compared to $J_{2}$, the reconstruction tendency is stronger than the layering ten- 
dency. Therefore, as the temperature rises, the layering critical points, $T=T_{c, n}$, will occur completely within the reconstructed phase. A single second-order reconstruction transition line will now enclose all of the layering transition lines (for sufficiently large $n$ ), terminating at the bulk surface rough-to-reconstructed-rough transition. The layering endpoints will accumulate at a lower temperature, $T=T_{r}$, corresponding to the bulk surface reconstructed-flat to reconstructed-rough transition. This scenario is pictured in Fig. 1(f). The numerical convergence of the mean-field theory becomes quite finicky in this regime of strongly negative $J_{1} / J_{2}$, hence the overly jagged antiferromagnetic line (better choices of parameters would improve this, but this regime is not our primary interest and we have not pursued such improvements).

Even more interesting behavior occurs if the effective coupling $K$ changes sign as a function of temperature at a value of $L$ larger than $L_{c}$. One may obtain phase diagrams that show both integer and half-integer layering. In Fig. 1(g) we show the case where $J_{1}$ is antiferromagnetic at low temperatures, turning ferromagnetic at high temperatures. The result is similar to that shown in Fig. 1(c), including a zigzagging line of first-order transitions that zip together the two sets of layering lines, differing only in that it is now the integer layering lines that are reentrant. In the bulk interface limit there are still two phase transitions. The surface is reconstructed at low temperatures, converts to the flat phase via a first-order transition at $T=T_{R}$, and finally roughens at $T=T_{r}$. The two sets of layering triple points, $T_{n}^{2}$ and $T_{n}^{1}$ must accumulate at the same point, $T=T_{R}$, because when $J_{1}=0$ only $J_{2}$ stabilizes the flat surface. For thick films $J_{2}$ does not distinguish between half-integer and integer layers, so the switch from one to the other must take place essentially over a vanishingly small temperature step.

In Fig. 1(h) we show what happens if $J_{1}$ is ferromagnetic at low temperatures and antiferromagnetic at higher temperatures. The possible behaviors are identical at high temperatures to those shown in Fig. 1(e) [or to those shown in Fig. $1(\mathrm{f})$ for a similar extension of path 5]. The only difference is that at low temperatures a new series of layering transitions between integer coverages takes over. These connect to the half-integer layering transitions in the same way as shown in Fig. 1(g), except that high and low temperatures are reversed. In the bulk interface limit there are now three transitions: a first-order transition from flat to reconstructed flat at low temperatures, followed by an Ising transition to the DOF phase, followed finally by a roughening transition to the film analogue of the reconstructed-rough phase (the last two would be reversed for the similar extension of path 5).

In neither of the two scenarios shown in Figs. $1(\mathrm{~g})$ and $(\mathrm{h})$ is preroughening involved because the reconstructed surface never disorders, but simply converts to the flat phase when $J_{1}$ changes sign. We emphasize these scenarios only because they mimic Fig. 1(c) but contain completely different physics. Figure 1(h) is especially similar since it is the halfinteger layering lines that are reentrant. In both phase diagrams, the first-order zipper appears. The difference now is that there is a higher-temperature Ising line below which the rough surface reconstructs. For the experiments that we will discuss, these scenarios are unlikely as there does not seem to be any indication that reconstruction takes place.
Although checkerboard reconstruction is described by the effective spin- $\frac{1}{2}$ Hamiltonian, the layering behavior discussed in the previous paragraphs is not since it involves three values of $n$. To derive the layering behavior from the RSOS model one must use at least a spin-1 Hamiltonian, which takes the general form

$$
\begin{aligned}
\overline{\mathcal{H}}_{1} \equiv & \frac{1}{2} K \sum_{\left\langle\mathbf{r r}^{\prime}\right\rangle}\left[s(\mathbf{r})-s\left(\mathbf{r}^{\prime}\right)\right]^{2}+\frac{1}{2} L \sum_{\left(\mathbf{r} \mathbf{r}^{\prime \prime}\right)}\left[s(\mathbf{r})-s\left(\mathbf{r}^{\prime \prime}\right)\right]^{2} \\
& -h \sum_{\mathbf{r}} s(\mathbf{r})+h_{2} \sum_{\mathbf{r}} s(\mathbf{r})^{2}
\end{aligned}
$$

where we have used the parabolic form, $-h s+h_{2} s^{2}$, with $h_{2}=H_{2} / k_{B} T$, to fit $V(h)$ for $h=n-1, n, n+1$, and dropped an overall constant $C_{1}=V(n) N_{A}$. Clearly the two parameters $h, h_{2}$ are all that are required. The restricted solid-onsolid (RSOS) condition now comes into play: since nearestneighbor sites can differ in height by at most unity, spin configurations in which $s(\mathbf{r})=+1$ and $s\left(\mathbf{r}^{\prime}\right)=-1$ for nearest-neighbor sites $\mathbf{r}$ and $\mathbf{r}^{\prime}$ are disallowed-in effect $K$ $=\infty$ for $\left|s(\mathbf{r})-s\left(\mathbf{r}^{\prime}\right)\right|=2$. Detailed computations of the layering behavior described in the previous paragraphs using this model will be described in Sec. IV.

\section{Film analogue of the DOF phase}

The spin- 1 model is also required to understand the film analogue of the DOF phase. Recall that preroughening involves a transition from a flat phase to a disordered reconstructed phase (i.e., a disordered flat phase). In the context of a thin film, the disordered flat phase will correspond to a checkerboard phase that has "melted," but nevertheless retains a preference for a certain density of atoms, namely a half-filled layer. To describe this properly the model must allow for two such phases: one with an extra half-layer on top of the flat phase, and one with a half-layer missing from the flat phase. This is crucial because it will turn out that these two phases arise from a kind of symmetry breaking in the flat phase. Given this, it is clear that three different layers enter the physics in a crucial way, and the effective layer Hamiltonian must allow three different values of the spin.

The disordered-flat phase on a bulk crystal interface occurs for small $K$ and moderate, but $L>0$ sufficiently large that $\left|h(\mathbf{r})-h\left(\mathbf{r}^{\prime \prime}\right)\right|=2$, where $\mathbf{r}$ and $\mathbf{r}^{\prime \prime}$ are second neighbors, is discouraged. The surface therefore is not rough, yet $K$ and $L$ are weak enough that $h(\mathbf{r})$ does not condense into a flat or reconstructed phase, preferring instead to take advantage of the entropy gain associated with a half-filled disordered layer. Clearly $J_{1}$ can have either sign, but we will be interested in $J_{1}>0$ so that the flat phase eventually stabilizes at low temperature. In the context of a thin film we are therefore asking the following question: if $H=0$ but $H_{2} \geqslant 0$ (so that $s=0$ is nominally preferred) are there conditions under which both $K$ and $L$ are positive (so that, again, $s=0$ is nominally preferred), and yet a spontaneously broken symmetry exists with $M \equiv\langle s(\mathbf{r})\rangle \neq 0$ ? Clearly the ground state of $\mathcal{H}_{1}$ under these conditions is $s(\mathbf{r}) \equiv 0$, but there may be an entropy driven transition to a state with $M \neq 0$ in some interval of temperatures. At high temperatures this symmetry breaking will be destroyed due to complete disordering of the film. At low temperatures it will be destroyed as energet- 


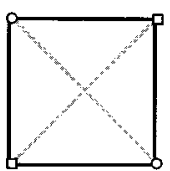

(a)

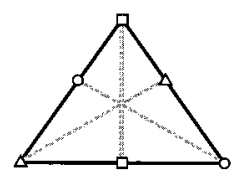

(b)

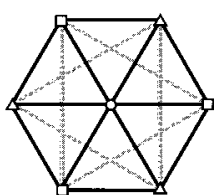

(c)
FIG. 6. (a) Four-spin plaquette for the square lattice containing two spins from each of the two sublattices. (b) Six-spin plaquette for the triangular lattice that violates the full rotational symmetry of the lattice, but treats the three sublattices symmetrically, keeping two spins from each. (c) Seven-spin plaquette for the triangular lattice that has the full rotational symmetry of the lattice, but breaks the symmetry between the three sublattices.

ics wins out over entropy. Although this scenario yields reentrant behavior of the type we seek, a calculation is required to see which of Figs. 1(a)-(d) give the correct global picture. Note that it is the absence of reconstruction in the DOF phase that eliminates the Ising line that is present in Fig. 1(h).

\section{Mean-field formalism}

The main calculational tool that we will use to explore the questions raised in the previous subsection is a selfconsistent mean-field formalism. The standard mean-field formalism replaces each individual fluctuating spin or height variable by an effective continuous single-site magnetization, or average height, which adjusts self-consistently to the effective field generated by its neighbors. Equivalently, the free energy is computed in a saddle-point approximation, with the phase space location of the saddle point determining the single-site magnetizations. Since all sites are equivalent in a ferromagnetic state, such an essentially single spin theory suffices to capture the basic physics. For antiferromagnetism on a square lattice, the two sublattices are inequivalent, but if the individual spins interact only with nearest neighbors there is no ambiguity in the local effective field. The single-spin mean-field theory then again suffices to capture the basic physics. However, if one wishes to describe ordering into a state involving subtle competition between correlations, one must improve the level of approximation by treating the fluctuations within plaquettes of nearby spins exactly. Interactions between different plaquettes are still treated self-consistently. The general formalism for doing this is outlined in Appendix A. In our case we are seeking a state that is formed by a delicate balance of nearest-neighbor and next-nearest-neighbor interactions. We therefore must keep enough spins that both types of interaction are present within a plaquette. For the square lattice we shall analyze a model using four spins in a given plaquette [see Fig. 6(a)]. For the triangular lattice we shall analyze two models, one with six-spin plaquettes [see Fig. 6(b)], and one with sevenspin plaquettes [see Fig. 6(c)].

\section{Square lattice}

In order to apply the mean-field formalism of Appendix A we need to tile the entire lattice with copies of the chosen plaquette, carefully distinguishing between intraplaquette and interplaquette interactions. This tiling is not unique, but

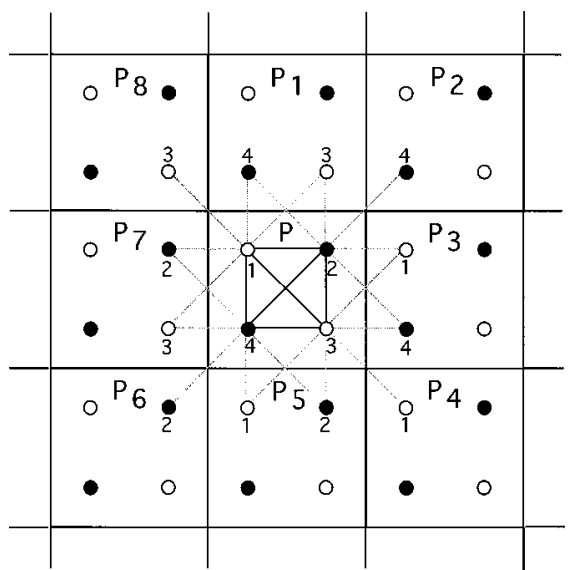

FIG. 7. A natural tiling of the full square lattice by the four-spin plaquette that maintains the symmetry of the lattice as well as the symmetry between the two sublattices.

for the square lattice plaquette there is a natural choice which is shown in Fig. 7. Let us begin by ignoring the RSOS constraint. Applying the formalism of Appendix A to the Hamiltonian (2.9), the single plaquette Hamiltonian corresponding to Fig. 7 is

$$
\begin{aligned}
\overline{\mathcal{H}}_{0}^{(4)}= & \frac{1}{2} K\left[\left(s_{1}-s_{2}\right)^{2}+\left(s_{2}-s_{3}\right)^{2}+\left(s_{3}-s_{4}\right)^{2}+\left(s_{4}-s_{1}\right)^{2}\right] \\
& +\frac{1}{2} L\left[\left(s_{1}-s_{3}\right)^{2}+\left(s_{2}-s_{4}\right)^{2}\right]+\widetilde{h}_{2}\left[s_{1}^{2}+s_{2}^{2}+s_{3}^{2}+s_{4}^{2}\right],
\end{aligned}
$$

where $\widetilde{h_{2}}=h_{2}+\lambda_{1} K+\frac{3}{2} \lambda_{2} L$, arising from multiplying out $\left(s_{i}-s_{j}\right)^{2}$ terms for $i$ and $j$ on different plaquettes. The interplaquette scale factors $\lambda_{1}$ and $\lambda_{2}$, nominally equal to unity, have been introduced for later convenience. Interactions between plaquettes then involve only products of pairs of single spins, so we need only introduce fields $H_{\alpha}$ conjugate to the individual spins, $\sigma_{\alpha}, \alpha=1,2,3,4$. Defining the single plaquette free energy $\Phi^{(4)}\left\{H_{\alpha}\right\}$ via Eq. (A5) we obtain the free-energy functional

$$
\begin{aligned}
\mathcal{F}^{(4)}\left\{H_{P \alpha} ; \sigma_{P \alpha}\right\}= & \sum_{P} \Phi^{(4)}\left\{H_{P \alpha}\right\}-\sum_{P \alpha}\left(H_{P \alpha}+h_{P \alpha}\right) \sigma_{P \alpha} \\
& -\lambda_{1} K \sum_{P}\left(\sigma_{P 1} \sigma_{P_{1} 4}+\sigma_{P 2} \sigma_{P_{1} 3}+\sigma_{P 2} \sigma_{P_{3} 1}\right. \\
& \left.+\sigma_{P 3} \sigma_{P_{3} 4}\right)-\lambda_{2} L \sum_{P}\left(\sigma_{P 1} \sigma_{P_{1} 3}\right. \\
& +\sigma_{P 2} \sigma_{P_{1} 4}+\sigma_{P 2} \sigma_{P_{2} 4}+\sigma_{P 2} \sigma_{P_{3} 4} \\
& \left.+\sigma_{P 3} \sigma_{P_{3} 1}+\sigma_{P 3} \sigma_{P_{4} 1}\right)
\end{aligned}
$$

where $P_{1}, P_{2}, P_{3}$, and $P_{4}$ are neighboring plaquettes to $P$ (see Fig. 7), and $\Phi^{(4)}$ will be computed explicitly in Sec. IV. Since the ordered phases we seek are all either ferromagnetic or antiferromagnetic we now take

$$
\begin{array}{cc}
h_{P 1}=h_{P 3} \equiv h_{A}, & h_{P 2}=h_{P 4} \equiv h_{B}, \\
H_{P 1}=H_{P 3} \equiv H_{A}, & H_{P 2}=H_{P 4} \equiv H_{B}, \\
\sigma_{P 1}=\sigma_{P 3} \equiv M_{A}, & \sigma_{P 2}=\sigma_{P 4} \equiv M_{B} .
\end{array}
$$


The free energy per spin is then

$$
\begin{aligned}
\frac{1}{N} \mathcal{F}^{(4)}\left(H_{A}, H_{B} ; M_{A}, M_{B}\right)= & \frac{1}{4} \Phi^{(4)}\left(H_{A}, H_{B}\right)-\frac{1}{2}\left(H_{A}+h_{A}\right) M_{A} \\
& -\frac{1}{2}\left(H_{B}+h_{B}\right) M_{B}-\lambda_{1} K M_{A} M_{B} \\
& -\frac{3}{4} \lambda_{2} L\left(M_{A}^{2}+M_{B}^{2}\right) .
\end{aligned}
$$

Differentiating with respect to $M_{A}$ and $M_{B}$ we obtain the first set of saddle-point conditions [see the first line of Eq. (A9)]

$$
M_{A}=\frac{1}{2} \frac{\partial \Phi^{(4)}}{\partial H_{A}}, \quad M_{B}=\frac{1}{2} \frac{\partial \Phi^{(4)}}{\partial H_{B}} .
$$

The mean-field free energy per spin is finally obtained by substituting these relations into Eq. (2.13) [this intermediate form represents the Bogoliubov free energy-see Eq. (A16)] and then minimizing over $H_{A}$ and $H_{B}$. This is equivalent to solving the second set of saddle-point equations [see the second line of Eq. (A9)]

$$
\begin{aligned}
& -H_{A}=2 \lambda_{1} K M_{B}+3 \lambda_{2} L M_{A}+h_{A}, \\
& -H_{B}=2 \lambda_{1} K M_{A}+3 \lambda_{2} L M_{B}+h_{B},
\end{aligned}
$$

where, again, Eq. (2.14) should be substituted for the $M_{A}$ and $M_{B}$ dependence. We emphasize that the order is important here: the alternative of using Eq. (2.15) first to eliminate $M_{A}$ and $M_{B}$ often leads to a free energy in which the saddle point is not a minimum. It is also worth commenting that, as discussed in Appendix A, consistency of the theory implies that the saddle-point conditions guarantee that

$$
\begin{aligned}
& M_{A}=\left\langle s_{P 1}\right\rangle=\left\langle s_{P 3}\right\rangle=-\frac{2}{N} \frac{\partial \mathcal{F}^{(4)}}{\partial h_{A}}, \\
& M_{B}=\left\langle s_{P 2}\right\rangle=\left\langle s_{P 4}\right\rangle=-\frac{2}{N} \frac{\partial \mathcal{F}^{(4)}}{\partial h_{B}} .
\end{aligned}
$$

This allows one to follow the alternative route of inverting Eq. (2.14) to eliminate $H_{A}$ and $H_{B}$ in favor of $M_{A}$ and $M_{B}$, and computing the Helmholtz free energy,

$$
\begin{aligned}
\frac{1}{N} A^{(4)}\left(M_{A}, M_{B}\right)= & \frac{1}{N} \mathcal{F}^{(4)}+\frac{1}{2}\left(h_{A} M_{A}+h_{B} M_{B}\right) \\
= & \frac{1}{4} \bar{\Phi}\left(M_{A}, M_{B}\right)-\frac{1}{2}\left[H_{A}\left(M_{A}, M_{B}\right) M_{A}\right. \\
& \left.+H_{B}\left(M_{A}, M_{B}\right) M_{B}\right]-\lambda_{1} K M_{A} M_{B} \\
& -\frac{3}{4} \lambda_{2} L\left(M_{A}^{2}+M_{B}^{2}\right),
\end{aligned}
$$

where $\bar{\Phi}^{(4)}\left(M_{A}, M_{B}\right)$ is obtained from $\Phi^{(4)}\left(H_{A}, H_{B}\right)$ through this elimination. The equilibrium magnetizations are then obtained via the equations of state,

$$
\frac{1}{2} h_{A}=\frac{1}{N} \frac{\partial A^{(4)}}{\partial M_{A}}, \quad \frac{1}{2} h_{B}=\frac{1}{N} \frac{\partial A^{(4)}}{\partial M_{B}} .
$$

The advantage here is that $A^{(4)}$ is a bona fide mean-field free energy depending only on the $M$ variables, and we avoid the "mixed" representation containing all three sets of variables, $h, H$, and $M$.

\section{RSOS condition}

Let us now turn to the inclusion of the RSOS condition. Recall that this condition requires that nearest-neighbor spins differ by at most one, implying a nearest-neighbor interaction $v_{R}\left(s-s^{\prime}\right)$ such that

$$
e^{-\beta v_{R}(s)}=\theta(1-|s|),
$$

where $\theta(x)$ is the step function [we take $\theta(0)=1$ ]. The condition is crucial for stabilizing the bulk crystal surface when $K<0$, since without it nearest-neighbor column height differences would diverge. Within a plaquette, i.e., in the computation of $\Phi$, this condition is easily accounted for simply by eliminating from the trace those spin configurations that violate it. However, between plaquettes greater care must be taken because one must now include the RSOS condition explicitly in the interplaquette interaction term $\mathcal{A}$ defined in Eq. (A1). The difficulty lies in the fact that $v_{R}(s)$ is not simply expressible as a polynomial in $s$. For integer values of $s, v_{R}(s)$ is the large $A$ limit of $v_{A}(s) \equiv A s^{2}\left(s^{2}-1\right)$. This form leads to new interaction terms $s_{i}^{2} s_{j}^{2}$ and $s_{i}^{3} s_{j}$. Unfortunately, within the mean-field approximation, the integer variable $s$ is replaced by a continuous variable $\sigma$, and the fact that $v_{A}(s) \rightarrow-\infty$ as $A \rightarrow \infty$ for $0<s^{2}<1$ leads to thermodynamic instabilities. The form $v_{A}(s)=A s^{2}\left(s^{2}-1\right)^{2}$ is healthier in this regard, but now involves even higher powers of the spins and still unphysically restricts the continuous variable $\sigma$ to the values $0, \pm 1$ when $A \rightarrow \infty$. One really needs $v_{A}(s)=A \theta(|s|-1)$, but this is nonpolynomial.

Our solution to this problem is to keep the RSOS condition within a plaquette, but "soften" it between plaquettes. The condition's main role is to discourage large nearestneighbor column height differences, and its exact form is a matter of convenience. We will consider then two "soft", forms for $v_{R}(s)$. Note that for $K>0$ it is safe to simply take $v_{R}(s) \equiv 0$, but for sufficiently large $K<0$ this choice becomes unstable to unbounded height differences between neighboring plaquettes. One solution then is to set $v_{R}(s)$ $=\left(\lambda_{1}(K)-1\right) K s^{2}$ [effectively replacing $K$ by $\lambda_{1}(K) K$ for all interplaquette interactions] with $0<\lambda(K) \leqslant 1$ a smooth function of $K$ that decreases as $K$ becomes more negative, thereby cancelling at least part of the nearest-neighbor interaction between plaquettes. At the same time one might enhance the interplaquette second-neighbor coupling, replacing $L$ by $\lambda_{2}(K) L$ with $\lambda_{2}(K)>1$. This allows $L$ to stabilize the reconstructed phase. It was precisely for this reason (as well as others-see below) that we introduced $\lambda_{1}$ and $\lambda_{2}$ in Eq. (2.11). Our second choice is to take $v_{R}(s)=A s^{4}$, with fixed $A>0$ of order unity chosen for convenience. This form guarantees thermodynamic stability without ad hoc variation of coefficients, at the expense of introducing higher powers of the spins. Unfortunately, it does allow ever larger nearestneighbor plaquette height differences as $K$ becomes more negative, violating the expected equivalence of all plaquettes. We have used precisely this form in computing the phase diagram shown in Fig. 1(f) (see Sec. IV A 2 for details), and the jaggedness of the antiferromagnetic line is probably due to all of these competing effects. Since our main focus is on ferromagnetic $J_{1}$ we have not seriously attempted to optimize our parameter choices here to improve this figure. 
One is actually led to considering linear rescalings of the interplaquette interactions for other reasons. For example, the relative number of nearest-neighbor and next-neighbor bonds internal to the plaquette in Fig. 6(a) (namely, 2:1) does not match the relative number in the full lattice (namely $1: 1$ ). One might, therefore, introduce phenomenological scale factors into the terms in (2.11) that couple to the environment, i.e., replace $K$ by $\lambda_{1} K$ and $L$ by $\lambda_{2} L$, and adjust $\lambda_{1}$ and $\lambda_{2}$ according to one's preference, or simply to optimize comparison with experiment.

It should now be clear how to write down spin- $j$ Hamiltonians for arbitrary $j$, even $j \rightarrow \infty$. Keeping more layers should improve the accuracy of the approximation for thicker films. Similarly, the construction of the mean-field theory is identical. The major differences are that the site free energy $\Phi$ becomes more complicated because there are more spin configurations to trace over.

\section{Triangular lattice}

The second-neighbor interaction divides the triangular lattice into three equivalent triangular sublattices, $A, B$, and $C$. We consider mean-field theories based on each of the two plaquettes of spins shown in Figs. 6(b) and 6(c). In the first, we keep two spins from each sublattice. In the second, we keep a hexagonal plaquette of seven spins that contains the full rotational symmetry of the triangular lattice, but unfortunately does not treat the three sublattices symmetrically: three spins are kept from each of two of the sublattices, but only one spin from the third. In neither case are all spins equivalent, which we will remedy somewhat by, again, introducing fudge factors $\lambda_{i}$ that scale the couplings to the surroundings.

In principle, to distinguish the three sublattices, we need three magnetic fields $h_{A}, h_{B}$, and $h_{C}$, with corresponding sublattice magnetizations $M_{A}, M_{B}$, and $M_{C}$. However, since even with negative $K$ frustration dictates that there are no phases that spontaneously break the symmetry between the three sublattices, we will keep only one field $h$ and assume the sublattice magnetizations to have all the same value $M$. Note that this is a statement about the exact behavior of the model. The mean-field approximation may well predict unphysical phases with broken symmetry. For this reason we will restrict triangular lattice computations to $K>0$. In discussing the effects of reconstructed phases we will always use a square lattice.

In order to apply the formalism of Appendix A we must again tile the plane with the basic plaquette. If one remains completely faithful to the triangular lattice, this turns out to be very unnatural. Examples of tilings are shown in Figs. 8 and 9. The hexagonal tiling maintains the rotational symmetry of the lattice, but has a "chirality,"' and therefore breaks the inversion symmetry. The triangular tiling is clearly highly nonunique, requires two different orientations of the basic plaquette, and breaks the rotational symmetry of the lattice more badly than does the triangle itself. The nonuniqueness reflects itself in the differing identifications of interplaquette and intraplaquette interactions implied by each possible tiling. For example, the symmetry of the triangle would normally imply equivalency of the three corner sites and equivalency of the three noncorner sites. However, in the tiling shown in Fig. 8 the top corner site connects to four

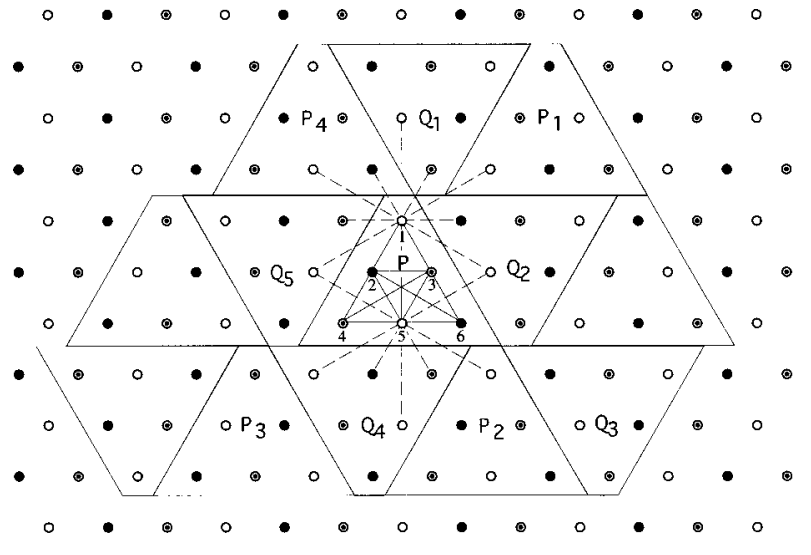

FIG. 8. A possible tiling of the full triangular lattice by the six-spin plaquette. Note that two different orientations of the original plaquette are required, and that the tiling is far from unique.

different plaquettes through nearest-neighbor bonds, while the right and left corner sites connect to three and to two different plaquettes, respectively. All six sites are therefore distinguishable and will have potentially different order parameter values. This is not only inconvenient for eventually solving the mean-field equations, but may also give rise to unphysical reconstructed phases. It seems clear that this will be true for any tiling with this plaquette.

Only by distorting the triangular lattice somewhat can one preserve the full symmetries of the plaquettes in the tiling: see Figs. 10 and 11. The drawback is that identifying second neighbors becomes ambiguous (see below). In particular, there is no way to preserve both the rotational symmetry and the property that second-neighbor bonds join sites only on the same sublattice. Notice in any case that both in Figs. 8,9 and in Figs. 10,11, different tiles contain different orientations of the sublattices $A, B$, and $C$, so any reconstructed phase that is uniform on each sublattice will not have the same periodicity as the tiling. A different choice of six-spin plaquette, say, would have to be made to respect this periodicity (for example, a parallelogram of two rows of three spins), but such a choice would generally violate the rota-

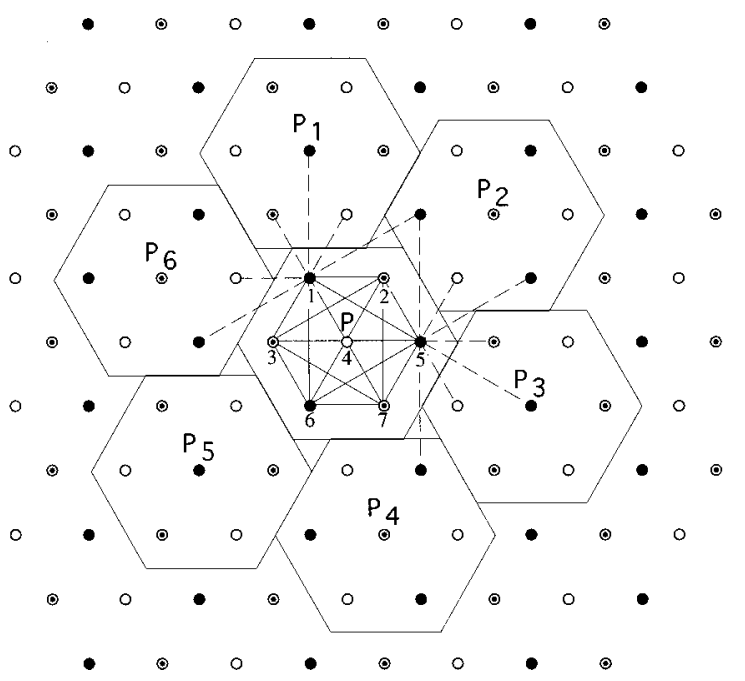

FIG. 9. A possible tiling of the full triangular lattice by the seven-spin plaquette. Other possible tilings differ only by translation or mirror reflection. 


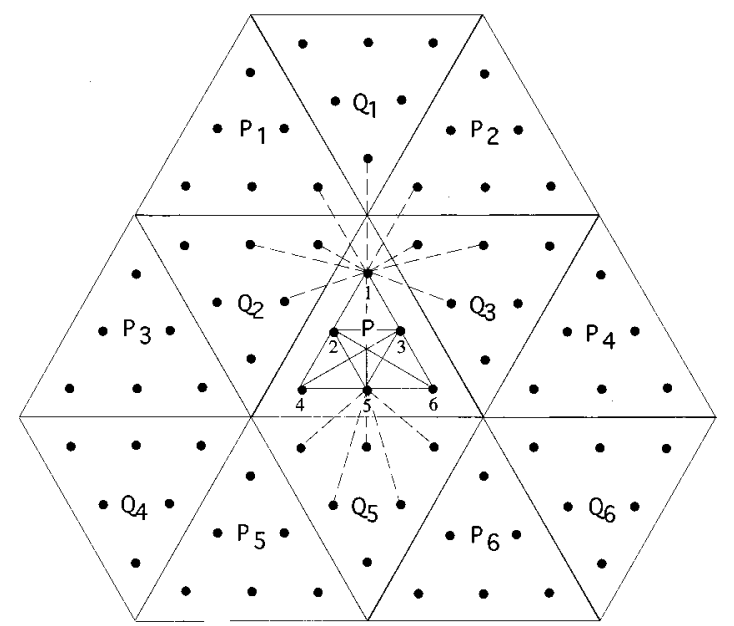

FIG. 10. A more symmetric tiling of a distorted triangular lattice by the six-spin plaquette. Two different orientations of the original plaquette are still required, but the tiling is unique up to translations. Choice of second-neighbor interactions becomes ambiguous, but unreconstructed phases should not be sensitive to this.

tional symmetry even further. Since we work only in the mean-field approximation and with ferromagnetic interactions we feel that maintenance of qualitative symmetries is more important than that of quantitative details of interactions. In any case, our hope is that the basic physics should be dominated by the interactions within the plaquette, which are treated exactly. We emphasize that we go through all this trouble of embedding the plaquette in a real lattice only to ensure that we obtain a fully consistent mean-field theory.

Keeping the above physical considerations in mind, we now write down the appropriate free energies. Detailed expressions and comparisons of the expressions obtained from the distorted and undistorted lattices are contained in Appendix B. Here we exhibit only the simplified expressions valid in the unreconstructed phases.

First, the single plaquette Hamiltonian corresponding to Fig. 6(b) is given by

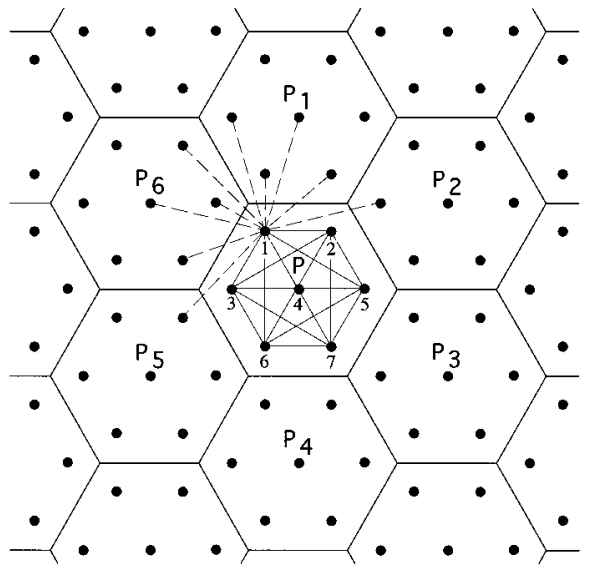

FIG. 11. A more symmetric tiling of a distorted triangular lattice by the seven-spin plaquette. The tiling is unique up to translations. Choice of second-neighbor interactions becomes ambiguous, but again unreconstructed phases should not be sensitive to this.

$$
\begin{aligned}
\overline{\mathcal{H}}_{0}^{(6)}= & \frac{1}{2} K\left[\left(s_{1}-s_{2}\right)^{2}+\left(s_{2}-s_{4}\right)^{2}+\left(s_{4}-s_{5}\right)^{2}+\left(s_{5}-s_{6}\right)^{2}\right. \\
& +\left(s_{3}-s_{6}\right)^{2}+\left(s_{1}-s_{3}\right)^{2}+\left(s_{2}-s_{3}\right)^{2}+\left(s_{3}-s_{5}\right)^{2} \\
& \left.+\left(s_{2}-s_{5}\right)^{2}\right]+\frac{1}{2} L\left[\left(s_{1}-s_{5}\right)^{2}+\left(s_{2}-s_{6}\right)^{2}\right. \\
& \left.+\left(s_{3}-s_{4}\right)^{2}\right]+\widetilde{h}_{2}^{\text {out }}\left[s_{1}^{2}+s_{4}^{2}+s_{6}^{2}\right] \\
& +\widetilde{h}_{2}^{\text {in }}\left[s_{2}^{2}+s_{3}^{2}+s_{5}^{2}\right],
\end{aligned}
$$

where $\tilde{h}_{2}^{\text {out }}=h_{2}+2 \lambda_{1} K+\frac{5}{2} \lambda_{2} L$ and $h_{2}^{\text {in }}=h_{2}+\lambda_{1} K+\frac{5}{2} \lambda_{2} L$. The scale factors, $\lambda_{1}$ and $\lambda_{2}$, have again been introduced for later convenience. Let $\Phi^{(6)}$ be the plaquette free energy defined in Eq. (A1) (to be computed explicitly in Sec. IV). Ignoring once again the RSOS condition between plaquettes, the free-energy functional corresponding to Fig. 10 is then (see Appendix B)

$$
\begin{aligned}
\frac{1}{N} \mathcal{F}^{(6)}\left(H_{\text {in }}, H_{\text {out }} ; M_{\text {in }}, M_{\text {out }}\right) \\
=\frac{1}{6} \Phi^{(6)}\left(H_{\text {in }}, H_{\text {out }}\right)-\frac{1}{2}\left[\left(H_{\text {out }}+h\right) M_{\text {out }}+\left(H_{\text {in }}+h\right) M_{\text {in }}\right] \\
\quad-\frac{1}{2} \lambda_{1} K\left(3 M_{\text {out }}^{2}+M_{\text {in }}^{2}+2 M_{\text {in }} M_{\text {out }}\right)-\lambda_{2} L\left(M_{\text {out }}^{2}+M_{\text {in }}^{2}\right. \\
\left.\quad+3 M_{\text {in }} M_{\text {out }}\right) .
\end{aligned}
$$

Here $M_{\text {out }}$ is the magnetization on the three corner sites of the plaquette, while $M_{\text {in }}$ is the magnetization on the three edge sites. Except for very special values of $\lambda_{1}$ and $\lambda_{2}$ the two will in general be different in the mean-field approximation. The same considerations apply to the fields $H_{\text {out }}$ and $H_{\text {in }}$.

Similarly, the plaquette Hamiltonian corresponding to the hexagonal plaquette in Fig. 6(c) is given by

$$
\begin{aligned}
\overline{\mathcal{H}}_{0}^{(7)}= & \frac{1}{2} K\left[\left(s_{1}-s_{2}\right)^{2}+\left(s_{2}-s_{5}\right)^{2}+\left(s_{5}-s_{7}\right)^{2}+\left(s_{7}-s_{6}\right)^{2}\right. \\
& +\left(s_{6}-s_{3}\right)^{2}+\left(s_{3}-s_{1}\right)^{2}+\left(s_{1}-s_{4}\right)^{2}+\left(s_{2}-s_{4}\right)^{2} \\
& \left.+\left(s_{3}-s_{4}\right)^{2}+\left(s_{5}-s_{4}\right)^{2}+\left(s_{6}-s_{4}\right)^{2}+\left(s_{7}-s_{4}\right)^{2}\right] \\
& +\frac{1}{2} L\left[\left(s_{1}-s_{6}\right)^{2}+\left(s_{1}-s_{5}\right)^{2}+\left(s_{2}-s_{6}\right)^{2}\right. \\
& \left.+\left(s_{5}-s_{6}\right)^{2}+\left(s_{3}-s_{7}\right)^{2}\right] \\
& +\widetilde{h}_{2}^{\text {out }}\left[s_{1}^{2}+s_{2}^{2}+s_{3}^{2}+s_{5}^{2}+s_{6}^{2}+s_{7}^{2}\right]+\widetilde{h}_{2}^{\text {in }} s_{4}^{2},
\end{aligned}
$$

where $\widetilde{h}_{2}^{\text {out }}=h_{2}+\frac{3}{2} \lambda_{1} K+2 \lambda_{2} L$ and $\widetilde{h}_{2}^{\text {in }}=h_{2}+3 \lambda_{2} L$. If $\Phi^{(7)}$ is the corresponding plaquette free energy, the mean-field free energy corresponding to Fig. 11 is then (see Appendix B)

$$
\begin{aligned}
\frac{1}{N} \mathcal{F}^{(7)}\left(H_{\text {in }}, H_{\text {out }} ; M_{\text {in }}, M_{\text {out }}\right) \\
\quad=\frac{1}{7} \Phi^{(7)}\left(H_{\text {in }}, H_{\text {out }}\right)-\frac{1}{7}\left[6\left(H_{\text {out }}+h\right) M_{\text {out }}+\left(H_{\text {in }}+h\right) M_{\text {in }}\right] \\
\quad-\frac{9}{7}\left(\lambda_{1} K+\lambda_{2} L\right) M_{\text {out }}^{2}-\frac{6}{7} \lambda_{2} L M_{\text {in }} M_{\text {out }} .
\end{aligned}
$$

Here $M_{\text {out }}$ is the magnetization on the outer ring of sites, while $M_{\text {in }}$ is the magnetization on the inner site. Fields $H_{\text {in }}$ and $H_{\text {out }}$ are defined similarly. 


\section{SINE-GORDON PHENOMENOLOGY: FIRST-ORDER PREROUGHENING AND ZIPPERING}

In this section we develop a general, large length-scale "hydrodynamic' theory of the layering phase diagram. This will serve as a rigorous guide to the different classes of behavior available to the system. A full microscopic calculation is still required to determine the behavior of any given model. The plaquette mean-field formalism will be applied to this end in later sections.

The basic idea we exploit is that roughening and preroughening are large-scale phenomena, governed only by a few renormalized parameters. The small-scale structure of the surface (be it locally disordered, flat, or possibly even reconstructed) feeds into these parameters, but is otherwise irrelevant to the large-scale behavior. Of course, a phase transition in the local structure could preempt the onset of long-range roughening or preroughening correlations (for example, it might induce some kind of critical endpoint with the roughening or preroughening line then ending on a firstorder line), but we assume this not to be the case. Imagine, then, that the system is close to a roughening or preroughening transition so that the correlation length is very large. The way we would formally derive the large-scale theory is to perform some kind of renormalization-group transformation on the Hamiltonian of the system, iterating it until we enter the neighborhood of the fixed point that governs the transition (we will argue at the very end of Sec. IV, in fact, that the plaquette mean-field theory accomplishes at least part of this step). If we are not precisely at criticality, further iteration will move the Hamiltonian away from the fixed point once more, but along a very restricted set of paths. The point is that during the approach to the fixed point all irrelevant variables have decayed away. Only one (or perhaps two, as we shall see) relevant variables remain, and it is their eventual growth that moves the Hamiltonian away from the fixed point. However, the dimension of this "escape manifold" is just the number of relevant variables. If we then stop the renormalization process on some matching boundary, not too far from the fixed point, we may parametrize the final theory with these one or two renormalized variables.

\section{A. Sine-Gordon-type models}

In many problems the detailed analysis of the fixed-point region cannot be performed explicitly. The advantage in the present case is that this region may be characterized simply and completely by a sine-Gordon-type model:

$$
\overline{\mathcal{H}}_{\mathrm{SG}}=\int d^{2} r\left\{\frac{1}{2} K_{0}|\nabla h(\mathbf{r})|^{2}+V_{0}[h(\mathbf{r})]\right\}
$$

with

$$
V_{0}[h]=-y_{0} \cos [2 \pi h(\mathbf{r})]-u_{0} \cos [4 \pi h(\mathbf{r})]+V_{\text {sub }}[h(\mathbf{r})],
$$

where $h(\mathbf{r})$ represents a coarse grained continuous surface height field, $K_{0}$ is a partially renormalized surface stiffness, $y_{0}$ represents the the fundamental Fourier component of the partially renormalized atomic periodic modulation, $u_{0}$ is the next harmonic, and $V_{\text {sub }}[h]$ is a partially renormalized substrate potential. The fixed point is actually a fixed line on which only $K_{0}$ is nonzero, and the critical behavior has already been alluded to in Eq. (1.2). As we will discuss in detail below, for pure Kosterlitz-Thouless roughening we may set $u_{0}=0$, but in order to discuss preroughening we must sometimes keep $u_{0} \neq 0 .{ }^{4}$ All higher harmonics, however, are irrelevant and may be assumed to have decayed to zero in the neighborhood of the fixed line. The substrate potential grows steeper under renormalization, and the form (3.1) is valid only in the thick-film limit where $V[h]$ is extremely weak, so that the partially renormalized $V_{0}(h)$ is weak as well. Since $V[h]$ has power-law behavior (2.2) for large $h, V_{0}[h]$ will as well. The quadratic form,

$$
V_{\text {sub }}[h] \approx \frac{1}{2} \kappa_{0}\left[h-h_{0}(\Delta \mu)\right]^{2}, \quad h \ll h_{0}(\Delta \mu),
$$

[see also Eq. (2.4)] with a renormalized curvature $\kappa_{0}$ suffices for thick films. Huse ${ }^{16}$ has written down general functional recursion relations for any potential $V_{0}[h]$ and treated in detail the case $u_{0}=0$, i.e., the interplay between roughening and layering. Here we will extend key parts of that analysis to the preroughening regime $u_{0} \neq 0$. It will transpire that $u_{0}$ $>0$ and $u_{0}<0$ can yield very different behaviors, and this gives rise to very interesting physics in the layering phase diagram.

To formalize what we have said so far we write down the renormalization-group recursion relations for the Hamiltonian (3.1): ${ }^{16}$

$$
\begin{gathered}
\frac{d K}{d l}=\kappa^{2} / 2 K \Lambda^{4}+\left(4 \pi^{4} / K \Lambda^{4}\right) y^{2}+\left(64 \pi^{4} / K \Lambda^{4}\right) u^{2}, \\
\frac{d y}{d l}=(2-\pi / K) y+\left(4 \pi^{2} / K \Lambda^{2}\right) y u, \\
\frac{d u}{d l}=(2-4 \pi / K) u-\left(\pi^{2} / K \Lambda^{2}\right) y^{2}, \\
\frac{d \kappa}{d l}=2 \kappa-\kappa^{2} / K \Lambda^{2},
\end{gathered}
$$

where $\Lambda \sim \pi / a$ is the (nonuniversal) momentum space cutoff due to the lattice. The flow parameter $l$ is related to the spatial rescaling factor $b$ via $b=b_{0} e^{l}$, where $b_{0}$ is the initial rescaling factor required to enter the neighborhood of the fixed line and is assumed to depend smoothly on the parameters of the initial RSOS model, say. The recursion relations are valid for small $y, u$, and $\kappa$ and we have the initial conditions $K(l=0)=K_{0}, \quad y(l=0)=y_{0}, \quad u(l=0)=u_{0}$, and $\kappa(l=0)=\kappa_{0}$, which are assumed to lie on some trajectory incoming toward the fixed line.

\section{B. Roughening and preroughening}

Let us now consider the various possible behaviors as a function of the initial condition. Consider first the substrate free case, $\kappa_{0}=0$. For small enough $K_{0} \quad\left(K_{0} \lesssim \pi / 2\right.$ for small $y$ and $u$ ) both $y$ and $u$ flow to zero as $l \rightarrow \infty$, while the stiffness $K(l) \rightarrow K_{R}\left(K_{0}\right)$, its fully renormalized value, which then appears in Eq. (1.2). This corresponds to the rough phase.

For intermediate values of $K_{0}\left(\pi / 2 \leqq K_{0} \leqq 2 \pi\right.$ for small $y$ and $u) u(l)$ still flows to zero, and may be ignored, but if 
$y_{0} \neq 0, y(l)$ eventually begins to grow again, as does $K(l)$. The strengthening corrugation potential, and increasing surface stiffness, signal the onset of a flat phase. Notice that if $y_{0}>0$ the minima of the corrugation potential occur at integer $h$, while if $y_{0}<0$ they occur at half-integer $h$. Since $y_{0}\left(J_{1}, J_{2}, T\right)$ is a renormalized parameter we may, in fact, imagine that as a result of short scale fluctuations it might change sign. The minima then switch abruptly from integer to half-integer. This precisely describes the physics of preroughening, with the preroughening critical line corresponding to $y_{0}(T)=0 .{ }^{4}$ The sign reversal is driven precisely by the entropy of small-scale roughness discussed in previous sections. As we shall see below, a negative value of $y_{0}$ could also be associated with a reconstructed surface, which may also roughen while maintaining a form of long-range reconstructed order. The sine-Gordon Hamiltonian does not distinguish between these two cases, though the dependence of the partially renormalized parameters on the original model parameters would of course be different (possibly even singular if a surface reconstruction transition takes place). For $y_{0}$ $=0$ the fixed line is again stable, and we will have $u(l) \rightarrow 0$ and $K(l) \rightarrow K_{R}$, with $\pi / 2<K_{R}<2 \pi$. The critical surface is therefore rough, but with a larger renormalized stiffness than is generically possible: the short-range fluctuations have renormalized away the strongest Fourier component of the corrugation potential.

Finally, for even larger $K_{0}\left(K_{0} \gtrsim 2 \pi\right.$ for small $y$ and $\left.u\right)$ both $y$ and $u$ are relevant, so even if $y_{0}=0$ the second harmonic of the corrugation potential will grow and the surface will flatten. Notice then that there are twice as many minima. This will be discussed in detail below. In principle, if we had a second free parameter at our disposal, we might imagine that both $y_{0}$ and $u_{0}$ could be made to vanish. Flattening would then take place only when the third harmonic became relevant, i.e., for $K_{0}>9 \pi / 2 .{ }^{21}$ This situation, however, does not seem to be experimentally relevant. If $y_{0}$ is not precisely zero then both $u$ and $y$ will grow under renormalization, and the interesting question then arises of how the two Fourier components might constructively or deconstructively interfere in the final renormalized corrugation potential. We shall explore these effects in detail below, seeing that they have very strong effects on both the surface and layering phase diagrams.

\section{Roughening and layering}

Since $u$ is strongly irrelevant for $K_{0} \lesssim 2 \pi$, the asymptotic behavior in the roughening and preroughening regions may be addressed simply by setting $u=0$ in the recursion relations, Eq. (3.4). The usual roughening transition may then be described by studying the region where the starting manifold $\left[y_{0}(T), K_{0}(T)\right]$ crosses the critical trajectory into the fixed point at $y=0, K=\pi / 2$. For small $y$ and $\lambda \equiv 2-\pi / K$ this trajectory is defined by $\lambda=\tilde{y}$, where $\tilde{y} \equiv\left(4 \sqrt{2} \pi / \Lambda^{2}\right) y$. Correct to quadratic order in $\lambda$ and $\tilde{y}$, the recursion relations simplify to

$$
\frac{d \kappa}{d l}=2 \kappa ; \quad \frac{d \lambda}{d l}=\widetilde{y} ; \quad \frac{d \tilde{y}}{d l}=\lambda \tilde{y} .
$$

The flows generated by these equations are shown in Fig. 12 .

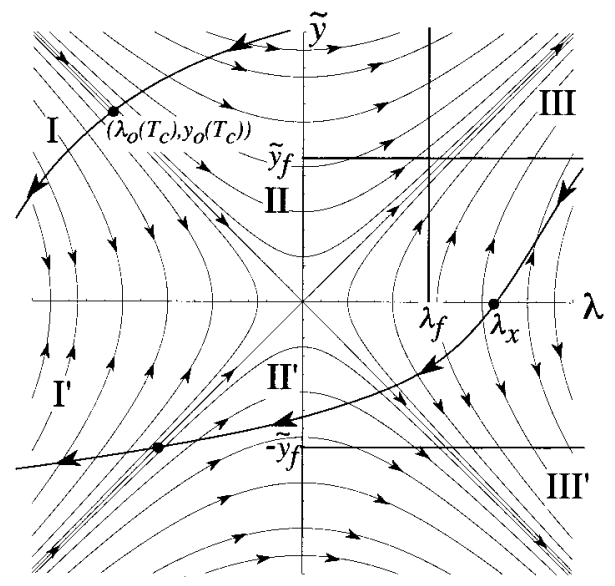

FIG. 12. Renormalization-group flows generated by Eqs. (3.5). Regions I, II, and III are bounded by the two separatrices that flow into and out of the Kosterlitz-Thouless fixed point at $\tilde{y}=\lambda=0$. The two thick lines represent possible physical starting manifolds, with the arrows indicating increasing temperature. The upper-left path corresponds to conventional roughening while the lower path represents preroughening [at the point $\left.\left(\lambda_{x}, 0\right)\right]$ followed by roughening. The solid lines at $\lambda=\lambda_{f}$ and $\tilde{y}=\tilde{y}_{f}$ represent the two possible noncritical matching manifolds, discussed in the text, at which the trajectory integration is stopped.

By integrating the flows in region II of this figure, from the starting manifold to some noncritical matching manifold, for example $\lambda=\lambda_{f}>0$, Huse ${ }^{16}$ has shown that the Ising layering critical points $T_{c, n}$ approach the bulk roughening temperature $T_{r}$ from below asymptotically as

$$
T_{r}-T_{c, n} \propto \frac{4 \pi^{2}}{(2+\alpha)^{2} \ln ^{2}(n / \tilde{n})},
$$

where $\alpha$, defined below Eq. (2.2), describes the power-law tail of the substrate potential, $\tilde{n}$ is a nonuniversal amplitude determined by the strength of the substrate potential, and the overall constant of proportionality depends on the detailed mapping of the origin model onto the sine-Gordon model.

\section{Preroughening and reentrant layering}

Preroughening, on the other hand, corresponds to the rather different situation in which the starting manifold begins in region III of Fig. 12. As the temperature rises the manifold crosses $\tilde{y}=0$ into region $\mathrm{III}^{\prime}$ at some positive value $\lambda_{0}$ of $\lambda$. Precisely at $\tilde{y}=0$ the system is on the fixed line and the interface is rough. On either side of $\tilde{y}=0$ the renormalization-group trajectories move away from the fixed line into an ordered phase. As before, region III corresponds to the flat phase. Region III $^{\prime}$ corresponds to the DOF phase. Since $\tilde{y}<0$ in the DOF phase, the minima in the corrugation potential occur at half-integer $h$. The fractional filling $\theta$ of the top layer of the interface then jumps discontinuously from $\theta=0$ to $\theta=\frac{1}{2}$ at preroughening. As the temperature continues to rise, the trajectory eventually crosses into region II' $^{\prime}$ and then into region I'. The latter corresponds to the transition from the DOF to the rough phase. In the presence of a substrate potential regions II' $^{\prime}$ and III' $^{\prime}$ give rise to first- 
order layering transitions between half-integer coverages. The Huse ${ }^{16}$ computation for the critical points goes through in exactly the same way and leads once again to Eq. (3.6) for the $T_{c, n}$ [see Fig. 1(b)].

The effect of the substrate on preroughening is quite different. The bulk critical behavior is now determined by the rate at which flows are pushed away from the fixed line for small $\tilde{y}$. This is completely determined by the value $\lambda_{X}$ at which the starting manifold crosses the $\tilde{y}=0$ axis. In particular, $\tilde{y}$ itself now plays the role of the deviation from criticality, the Kosterlitz-Thouless fixed point no longer plays any role, and the flows are completely confined to regions III and III'. The solutions to the flow equations in these regions are given by

$$
\begin{gathered}
\kappa(l)=\kappa_{0} e^{2 l}, \quad \tilde{y}(l)=-\operatorname{sgn}\left(\widetilde{y}_{0}\right) B_{0} \operatorname{csch}\left(B_{0} l+\phi_{0}\right), \\
\lambda(l)=-B_{0} \operatorname{coth}\left(B_{0} l+\phi_{0}\right),
\end{gathered}
$$

where

$$
B_{0}^{2}=\lambda_{0}^{2}-\widetilde{y}_{0}^{2}=\lambda(l)^{2}-\tilde{y}(l)^{2}>0, \quad \phi_{0}=\frac{1}{2} \ln \left(\frac{\lambda_{0}-B_{0}}{\lambda_{0}+B_{0}}\right)<0 .
$$

We run the flows until $|\tilde{y}(l)|=\tilde{y}_{f}$, some fixed value. The corresponding value $l_{f}$ of $l$ is then

$$
\begin{aligned}
l_{f} & =-\frac{1}{B_{0}} \sinh ^{-1}\left(B_{0} / \tilde{y_{f}}\right)-\frac{\phi_{0}}{B_{0}} \\
& \approx-\frac{1}{\lambda_{X}} \sinh ^{-1}\left(\lambda_{X} / \widetilde{y_{f}}\right)-\frac{1}{\lambda_{X}} \ln \left(\left|\tilde{y}_{0}\right| / \lambda_{X}\right),
\end{aligned}
$$

where the second line is valid for $\widetilde{y_{0}} \ll \lambda_{0}$. At this point we have

$$
\lambda_{f} \equiv \lambda\left(l_{f}\right)=\sqrt{B_{0}^{2}+\widetilde{y}_{f}^{2}} \approx \sqrt{\lambda_{X}^{2}+\widetilde{y}_{f}^{2}}
$$

Following Huse, ${ }^{16}$ for given values of $\tilde{y}$ and $\lambda$, there will be a critical value of $\kappa=\kappa_{c}(\tilde{y}, \lambda)$ at which the Ising layering critical point occurs. Let us define

$$
\kappa_{c}^{ \pm}\left(\lambda_{X}\right)=\kappa_{c}\left( \pm \widetilde{y_{f}}, \sqrt{\lambda_{X}^{2}+\widetilde{y}_{f}^{2}}\right) .
$$

Then, as $\widetilde{y} \rightarrow 0$, we locate the value of $\kappa_{0}$ at which the critical point occurs by demanding that

$$
\kappa_{c}\left( \pm \tilde{y}_{f}, \lambda_{f}\right) \approx \kappa_{c}^{ \pm}\left(\lambda_{X}\right)=\kappa_{0} e^{2 l_{f}}, \quad \pm \tilde{y_{0}}>0,
$$

which yields

$$
\kappa_{0} \approx \widetilde{\kappa}^{ \pm}\left(\lambda_{X}\right)\left(\left|{\widetilde{y_{0}}}\right| / 2 \lambda_{X}\right)^{2 / \lambda_{X}}
$$

where

$$
\widetilde{\kappa}^{ \pm}\left(\lambda_{X}\right) \equiv \kappa_{c}^{ \pm}\left(\lambda_{X}\right) e^{\left(2 / \lambda_{X}\right) \sinh ^{-1}\left(\lambda_{X} / \tilde{y}_{f}\right)} .
$$

Finally, from Eq. (2.3) for a van der Waals substrate we have $\kappa_{0}=\alpha(\alpha+1) c / h_{0}(\Delta \mu)^{2+\alpha}$, with the $n$th layering line corresponding to $h_{0}(\Delta \mu)=n-\frac{1}{2}$ for $\widetilde{y_{0}}>0$ and to $h_{0}(\Delta \mu)=n$ for $\widetilde{y_{0}}<0$ (i.e., exact degeneracy of two neighboring minima in the renormalized corrugation potential). This yields immediately [see Fig. 1(b)]

$$
\begin{array}{cc}
T_{\mathrm{pr}}-T_{n}^{1} \propto \tilde{y_{0}} \approx 2 \lambda_{X}\left(\tilde{n}^{+} / n\right)^{(2+\alpha) \lambda_{X} / 2}, & \tilde{y_{0}}>0 \\
T_{n}^{2}-T_{\mathrm{pr}} \propto-\tilde{y_{0}} \approx 2 \lambda_{X}\left(\tilde{n}^{-} / n\right)^{(2+\alpha) \lambda_{X} / 2}, \quad \tilde{y_{0}}<0,
\end{array}
$$

where $\widetilde{n}^{ \pm}\left(\lambda_{X}\right)=\left[\alpha(\alpha+1) c / \widetilde{\kappa}^{ \pm}\left(\lambda_{X}\right)\right]^{1 /(2+\alpha)}$ is a nonuniversal amplitude. Once again the overall constants of proportionality are determined by the detailed mapping of the original model onto the sine-Gordon model. We see then that the critical points have a power-law rather than logarithmic approach to the preroughening point. The power is nonuniversal, depending on $\lambda_{X}$, and vanishes as the KosterlitzThouless point is approached. We have therefore established Fig. 1(b) as the correct thick-film layering phase diagram corresponding to a preroughening trajectory such as that shown in Fig. 12.

\section{E. Recursion relations when $u$ is relevant}

We have seen that the experimental phase diagrams for argon and krypton on graphite show rather different behavior, with apparent first-order lines that "zip" the integer and half-integer layering lines together. It is possible that these transitions arise from some confluence of preroughening and two-dimensional melting phenomena, where the melting and preroughening temperatures are nearly the same. This is certainly true in the first two layers where two-dimensional triple points are observed. ${ }^{12,13}$ However, it seems an unlikely coincidence that such a confluence would survive, as seen, to much thicker films, where the energetics of melting and preroughening ought to be distinct. Here we offer a much simpler and more natural explanation, phrased entirely within the physics of the sine-Gordon model. More detailed comparisons between theory and experiment will be made in Sec. V.

The idea now is to consider values of $K_{0}$ in the region where $u$ becomes relevant. Typically, $u$ will be of order unity in the original model, so if $K_{0}$ is significantly larger than $2 \pi$ then even when $y \equiv 0$ the renormalization-group flows will never come close to the fixed line, and there will be no simple analytic description of the behavior. We therefore assume that $K_{0}$ is sufficiently close to $2 \pi$ that, in the absence of $y, u_{0}$ may be assumed small. Defining $\bar{y}$ $=\sqrt{2} \pi y / \Lambda^{2}, \quad \bar{u}=4 \sqrt{2} \pi u / \Lambda^{2}$ and $\mu=2-4 \pi / K$, correct to quadratic order in these variables the recursion relations (3.4) simplify to

$$
\begin{gathered}
\frac{d \kappa}{d l}=2 \kappa, \quad \frac{d \bar{y}}{d l}=\frac{3}{2} \bar{y}+\frac{1}{4} \mu \bar{y}+\frac{\sqrt{2}}{4} \bar{u} \bar{y}, \\
\frac{d \mu}{d l}=\bar{y}^{2}+\bar{u}^{2}, \quad \frac{d \bar{u}}{d l}=\mu \bar{u}-\sqrt{2} \bar{y}^{2} .
\end{gathered}
$$

If $\bar{y}_{0} \ll u_{0}$ these further simplify to 


$$
\frac{d \kappa}{d l}=2 \kappa ; \quad \frac{d \mu}{d l}=\bar{u}^{2} ; \quad \frac{d \bar{u}}{d l}=\mu \bar{u} ; \quad \frac{d \bar{y}}{d l}=\frac{3}{2} \bar{y} ;
$$

the first three of which are identical to Eq. (3.4) with $\mu$ replacing $\lambda$ and $\bar{u}$ replacing $\tilde{y}$. The solutions, in the equivalent to region II, are

$$
\begin{gathered}
\kappa(l)=\kappa_{0} e^{2 l}, \quad y(l)=y_{0} e^{3 / 2} l \\
\bar{u}(l)=A_{0} \sec \left(A_{0} l+\theta_{0}\right), \quad \mu(l)=A_{0} \tan \left(A_{0} l+\theta_{0}\right)
\end{gathered}
$$

with $A_{0}^{2}=\bar{u}_{0}^{2}-\mu^{2}>0$ and $\theta_{0}=\tan ^{-1}\left(\mu_{0} / A_{0}\right)$. These solutions hold up until $\bar{y} \approx \bar{u}$. In the absence of $\bar{y}_{0}$ we would integrate these equations until $\mu=\mu\left(l_{f}^{0}\right) \equiv \mu_{f}^{0}>0 \quad$ [and $\left|\bar{u}\left(l_{f}^{0}\right)\right|=\vec{u}_{f}^{0}$ where $\left.\left(\bar{u}_{f}^{0}\right)^{2}=A_{0}^{2}+\left(\mu_{f}^{0}\right)^{2}\right]$ reaches some final value [just as in Huse's analysis of Eq. (3.5)]. If $\bar{y}_{0}$ were to remain zero for all $\mu_{0}$ (or, equivalently, $K_{0}$ ), we would then predict, as a function of $\mu_{0}$, first-order layering lines terminating in Ising critical points every half-layer. However, since $\bar{y}_{0}$ vanishes only at the putative preroughening point, we conclude that there is only a single value of $\mu_{0}$ at which this analysis is correct. Since we assume the model to be in region II, rather than region $I$, the bulk interface would be in the flat phase. In thick films we would therefore observe first-order transitions every half-layer, with Ising critical points observed only, perhaps, for an initial finite set of layers (the closer the initial values to the incoming separatrix, the greater the number of critical points).

What happens away from this value of $\mu_{0}$ depends upon the growth of $y_{0}$ under renormalization. If $y_{0}$ is so small that $\bar{y}_{f}^{0} \equiv \bar{y}_{0} \exp \left(\frac{3}{2} l_{f}^{0}\right) \ll \bar{u}_{f}^{0}$, then we may still use Eq. (3.18), and stop integrating at $l_{f}^{0}$ as before. Thus, as $y_{0}$ passes through zero, the contribution $y_{f}^{0}$ of the lowest harmonic to the corrugation potential is linear in $y_{0}$. If, however, $\bar{y}_{f} \gtrsim \bar{u}_{f}$ then we should stop integrating at $l_{f}$ such that $\bar{y}\left(l_{f}\right) \equiv \bar{y}_{f} \approx \mu_{f}^{0}$, say, some final value. There is then a regime in the integration where $\bar{y}(l) \gtrsim \bar{u}(l)$, and the solutions (3.18) are no longer valid. If $\bar{u}(l)$ is not too much smaller than $\bar{y}_{f}$ we may use the fact that $\bar{y}$ is rapidly varying relative to $\bar{u}$ and $\mu$. Thus in the time it takes $\bar{y}(l)$ to go from $\bar{u}(l)$ to $y_{f}$ it is easy to see that $\bar{u}(l)$ and $\mu(l)$ change only by $O\left(y_{f}^{2}\right)$, which we assume to be much smaller than $\bar{u}(l)$. Thus $\bar{u}_{f}$ and $\mu_{f}$ are essentially the unperturbed values of $\mu$ and $\bar{u}$ at which $y(l)$ "crosses", $\bar{u}(l)$. If, on the other hand, $\bar{u}(l)$ and $\mu(l)$ are very small compared to $\bar{y}_{f}$, then we may essentially delete all but $\bar{y}(l)$ from the right-hand sides of (3.16): the flows are driven entirely by $\bar{y}(l)$. The final values, $\bar{u}_{f}$ and $\bar{\mu}_{f}$, are then of order $y_{f}^{2} \ll y_{f}$.

To summarize, then, we are interested in the final renormalized form of the corrugation potential. The above analysis shows that for small $\bar{y}_{0}$, the amplitude of the fundamental Fourier component varies linearly with $\bar{y}_{0}$ and changes sign precisely when $\bar{y}_{0}$ does, while the amplitude of the second harmonic can be taken as fixed. For larger $\bar{y}_{0}$, the ratio of the amplitudes, $\bar{y}_{f} / \bar{u}_{f}$, is nonlinear, but monotonically increasing in $\bar{y}_{0}$. This is all we need to know for the purposes of the following analysis.

\section{F. Thermodynamics of the bulk interface when $u$ is relevant}

Now that we have understood the general structure of the fully renormalized Hamiltonian, we must understand its thermodynamics. We are in a regime in which the corrugation potential wins out over thermal fluctuations, leading to a flat phase in which the interface height sits at a minimum of the potential. Since thermal fluctuations have not been completely integrated out $\left[K_{f}=4 \pi /\left(2-\mu_{f}\right)\right.$ is still finite-this was necessitated by the restricted regime in which the flow equations are valid] this is not entirely accurate: the interface still has fluctuations about this minimum. Since $K_{f}$ is large, however, these fluctuations may be taken as small (so long as one is not too close to any second-order phase transitionsee further below), leading to some slight renormalization of the corrugation potential, but not altering its basic form. Including the substrate potential, we therefore arrive, essentially rigorously, at the following single variable free-energy functional, which completely determines the thermodynamics:

$$
f[h]=-y_{R} \cos (2 \pi h)-u_{R} \cos (4 \pi h)+\frac{1}{2} \kappa_{R}\left(h-h_{0}\right)^{2},
$$

where the absolute minimum of $f(h)$ determines the equilibrium average interface height, and $y_{R}$ and $u_{R}$ are mildly renormalized versions of $y_{f} \equiv\left(\Lambda^{2} / \sqrt{2} \pi\right) \bar{y}_{f}$ and $u_{f}$ $\equiv\left(\Lambda^{2} / 4 \sqrt{2} \pi\right) \bar{u}_{f}$ into which $K_{f}$ has been completely subsumed. Similarly for $\kappa_{R} \approx \kappa_{f} \equiv \kappa_{0} \exp \left(2 l_{f}\right)$, where we assume that $\kappa_{0}$ is sufficiently small that $l_{f}$ is set only by the bulk interface recursion relations. This means, for example, that $\kappa_{R}$ is linearly related to $\kappa_{0}$. We reiterate that the validity of this free energy presumes that the essential physics lies only in the large-scale, coarse-grained fluctuations. It is also possible that small scale energetics of the original model preempt this physics at some temperature, beyond which Eq. (3.19), and the entire sine-Gordon analysis, fails (see further below). The control variable is $y_{R}$, which switches sign, while $u_{R}$ may be taken as fixed and nonzero, but either positive or negative.

\section{1. $u_{R}>0$ : first-order preroughening and zippering}

Begin with the bulk interface, $\kappa_{R} \equiv 0$. Suppose first that $u_{R}>0$, and imagine beginning with $y_{R} \gg u_{R}$, then decreasing $y_{R}$ through zero, and ending with $y_{R} \ll-u_{R}$. The evolution of the corrugation potential is shown in Fig. 13(a). We see that when $y_{R}=4 u_{R}$ local minima develop at half-integer $h$. Since these local minima are not absolute minima, the surface height remains an integer. As $y_{R}$ decreases these local minima decrease, and precisely at $y_{R}=0$ they become degenerate with the integer minima. For $y_{R}<0$ the half-integer minima lie below the integer minima, and therefore define the equilibrium surface height. We therefore have a firstorder transition from the flat to the DOF phase. The preroughening line therefore has a tricritical point precisely where the fully renormalized stiffness reaches $2 \pi$. 
(a)

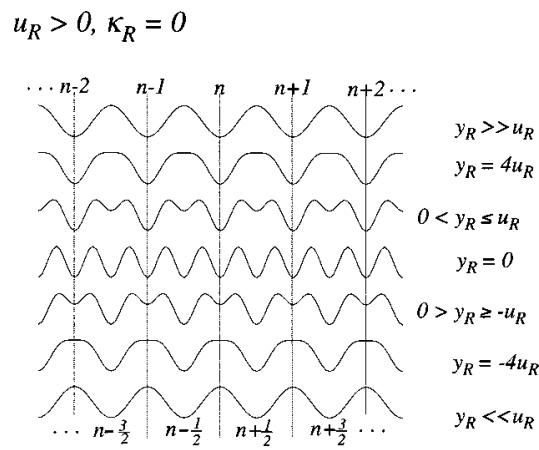

(b)

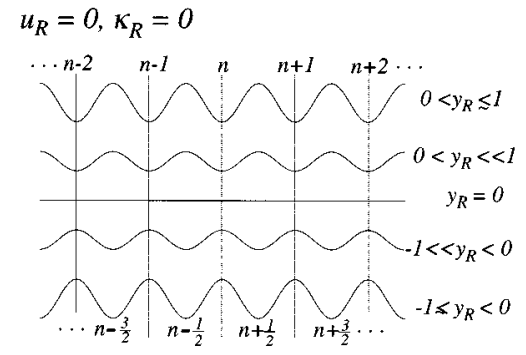

FIG. 13. (a) Corrugation potential for the bulk interface $\left(\kappa_{R}\right.$ $=0)$ with $u_{R}>0$ as a function of $y_{R}$. There is a first-order preroughening transition at $y_{R}=0$ when the integer minima exchange stability with the half-integer minima. (b) For comparison, the corrugation potential in the continuous preroughening case, $u_{R}=0$.

For clarity, this picture is contrasted in Fig. 13(b) with the standard preroughening case in which $u_{R} \equiv 0$. There, at $y_{R}$ $=0$ the corrugation potential is competely flat and the interface is free to wander.

Consider now the addition of the substrate potential $\kappa_{R}$. Since $\kappa_{R}$ will vary only slowly with film thickness, our control variable is $h_{0}$. Minimizing Eq. (3.19) yields the equation

$$
\sin (4 \pi h)+\frac{y_{R}}{2 u_{R}} \sin (2 \pi h)=-\frac{\kappa_{R}}{4 \pi u_{R}}\left(h-h_{0}\right) .
$$

Suppose first that $y_{R}=0$, in which case we require

$$
\sin (4 \pi h)=-\frac{\kappa_{R}}{4 \pi u_{R}}\left(h-h_{0}\right) .
$$

By periodicity we may suppose that $n \leqslant h_{0} \leqslant n+\frac{1}{2}$. The local minima closest to $h_{0}$ that solve this equation lie just above $n$ and just below $n+\frac{1}{2}$. When $h_{0}=n+\frac{1}{4}$ they are symmetrically located and degenerate. They exist for sufficiently small $\kappa_{R}$, namely,

$$
\kappa_{R}<(4 \pi)^{2} u_{R} .
$$

Thus for sufficiently thick films we will have, with increasing $h_{0}$, a first-order transition precisely at $h_{0}=n+\frac{1}{4}$ from slightly more than $n$ layers to slightly less than $n+\frac{1}{2}$ layers. As $h_{0}$ increases further, $h$ will increase to slightly more than $n+\frac{1}{2}$ layers until, precisely at $h_{0}=n+\frac{3}{4}$, there is transition to slightly less than $n+1$ layers. If Eq. (3.22) is not satisfied the substrate wipes out the corrugation and the film will grow continuously until Eq. (3.22) is satisfied. The closer we are to the triple point, the larger will be $\kappa_{R}$ and the thicker the film required to see layering.
In the opposite limit, where $\left|y_{R}\right|>4 u_{R}$, we may ignore $u_{R}$ and obtain essentially the same picture as above, but with twice the period. Thus if $y_{R}>0$ there are first-order transitions between essentially integer interface heights precisely at $h_{0}=n+\frac{1}{2}$, while if $y_{R}<0$ the transitions are between essentially half-integer interface heights precisely at $h_{0}=n$. Both sets of transitions are wiped out unless $\kappa_{R}$ $<(2 \pi)^{2} y_{R}$.

The interesting question is what happens for $0<\left|y_{R}\right|$ $<4 u_{R}$. Clearly the local minima at half-integer $h$ are most stable if $h_{0}=n+\frac{1}{2}$. This minimum can be an absolute minimum only if $\kappa_{R}$ is sufficiently large, namely,

$$
\kappa_{R}>\kappa_{R}^{t} \equiv 16\left|y_{R}\right|\left[1+\frac{\left|y_{R}\right|}{\pi^{2} u_{R}}-\left(\pi^{2}-4\right) \frac{y_{R}^{2}}{4 \pi^{4} u_{R}^{2}}+O\left(\frac{\left|y_{R}\right|^{3}}{u_{R}^{3}}\right)\right]
$$

for small $\kappa_{R} / u_{R}$ and $y_{R} / u_{R}$, which will be valid for thick films close to the bulk first-order transition at $y_{R}=0$. If this inequality is violated, which will always occur for sufficiently thick flims, only the transitions between integer surface heights (for $y_{R}>0$ ) or half-integer surface heights (for $\left.y_{R}<0\right)$ will be observed. If the inequality is satisfied, both sets of transitions will be seen. For $\kappa_{R}$ larger than $\kappa_{R}^{t}$, we may compute the range, $\Delta h_{0}$, of $h_{0}$ around $n+\frac{1}{2} \quad\left(y_{R}>0\right)$ or $n\left(y_{R}<0\right)$ over which the new minimum is stable. Indeed one finds that

$$
\Delta h_{0}=\frac{1}{4}\left[1-\frac{2 y_{R}}{\pi^{2} u_{R}}+O\left(\frac{y_{R}^{2}}{u_{R}^{2}}, \frac{\kappa_{R}-\kappa_{R}^{t}}{u_{R}}\right)\right] \frac{\kappa_{R}-\kappa_{R}^{t}}{\kappa_{R}^{t}} .
$$

Thus $\kappa_{R}^{t}\left(y_{R}\right)$ is a triple point, with two new first-order transitions extending out linearly from the horizontal layering lines at larger $\left|y_{R}\right|$. At $y_{R}=0$ these new lines are precisely the transitions at $n \pm \frac{1}{4}$ found above. In the thick-film limit $\kappa_{R} \rightarrow 0$ one sees from Eq. (3.24) that these lines are essentially straight. We have therefore confirmed precisely the zippering picture shown in Fig. 1(c).

Finally, since $\kappa_{R} \sim 1 / n^{2+\alpha}$ and, inverting Eq. (3.23), the triple point position $y_{R}^{t}\left(\kappa_{R}\right) \approx \kappa_{R} / 16 u_{R}$ vanishes linearly with $\kappa_{R}$, the two sequences of triple points on either side of $y_{R}$ $=0$ converge to the bulk interface first-order preroughening temperature $T_{0}$ also as $\left|T_{n}^{1,2}-T_{f o}\right| \sim 1 / n^{2+\alpha}$ (with $\alpha=2$ for a van der Waals substrate potential).

To complete the analysis, we discuss the question of how Fig. 1(c) converts to Fig. 1(b), either as $\kappa_{R}$ increases or as $K_{0}$ decreases into the region where $u$ is irrelevant. We shall see that if $\kappa_{R} / u_{R}$ is sufficiently large, the triple points are wiped out and replaced by ordinary Ising critical points. We wish to understand how this happens in detail.

We have already seen that when $y_{R}=0$ the first-order transitions at $h_{0}=n \pm \frac{1}{4}$ disappear if $\kappa_{R} / u_{R}>(4 \pi)^{2}$. More generally, the critical point that signals the first appearance of the first-order transition occurs when the line representing the right-hand side of Eq. (3.20) precisely kisses an inflection point of the left-hand side [see Fig. 14(a)]. For $y_{R}=0$ this inflection point is at $h=n \pm \frac{1}{4}$, and the slope at this point gives the above critical value, $\kappa_{R} / 4 \pi u_{R}=k_{c}\left(y_{R}=0\right) \equiv 4 \pi$. For $y_{R} \neq 0$ but small one finds that the inflection points are at 
(a)

$\sin (4 \pi h)+\left(y_{R} 2 u_{R}\right) \sin (2 \pi h)=-\kappa_{R}\left(h-h_{o}\right) / 4 \pi u_{R}$

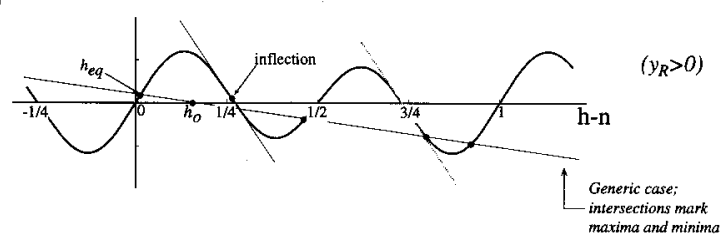

(b)

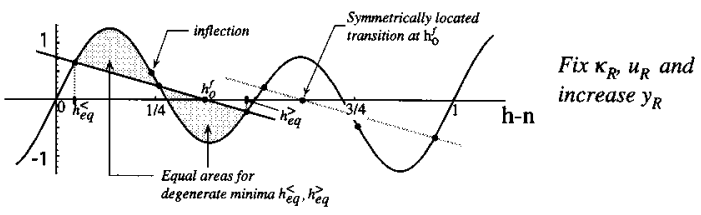

(c)

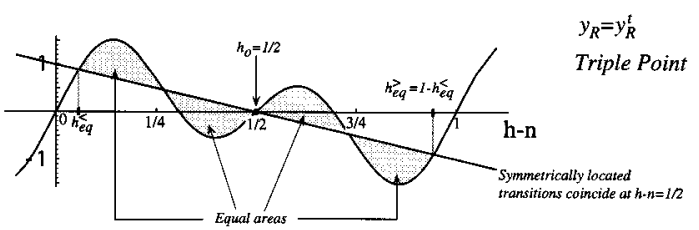

(d)

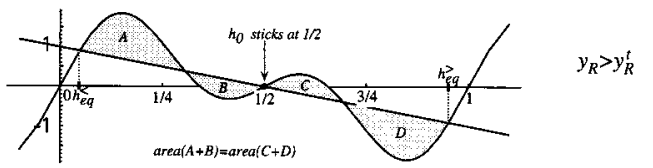

FIG. 14. Graphical solutions of Eq. (3.20) for $u_{R}>0$, which relates the layering phase diagram to the behavior of the bulk interface in the first-order preroughening regime. (a) Inflection points and the first appearance of first-order layering lines in the zipper regime. (b) Equal areas construction for the position of the firstorder zipper layering line. (c) The triple point. (d) Ordinary layering beyond the triple point.

$$
\pm\left(h_{i n f l}-n\right)=\frac{1}{4}+\frac{y_{R}}{32 \pi u_{R}}+\frac{2 \pi^{2}}{3}\left(\frac{y_{R}}{32 \pi u_{R}}\right)^{3}+O\left(\frac{y_{R}^{5}}{u_{R}^{5}}\right)
$$

and the (negative of the) slope is

$$
\begin{aligned}
k_{c}\left(y_{R}\right)= & 4 \pi\left[1+8 \pi^{2}\left(\frac{y_{R}}{32 \pi u_{R}}\right)^{2}+128 \pi^{4}\left(\frac{y_{R}}{32 \pi u_{R}}\right)^{4}\right. \\
& \left.+O\left(\frac{y_{R}^{6}}{u_{R}^{6}}\right)\right]
\end{aligned}
$$

with corresponding value of $h_{0}$,

$$
\pm\left(h_{0}^{c}-n\right)=4 \frac{y_{R}}{32 \pi u_{R}}-\frac{88 \pi^{2}}{3}\left(\frac{y_{R}}{32 \pi u_{R}}\right)^{3}+O\left(\frac{y_{R}^{5}}{u_{R}^{5}}\right) .
$$

This is larger than $k_{c}(0)$, meaning that the first-order transition is more stable for $y_{R} \neq 0$, existing for larger $\kappa_{R} / u_{R}$. Note that this computation assumes, effectively, $K_{R} \rightarrow \infty$ so that mean-field theory is exact. This is fine for first-order transitions, but for second-order transitions there will, in fact, be fluctuation corrections to this behavior so that both the exact position of the critical point and the critical behavior (which will be that of the two-dimensional Ising model) will be different. (a)

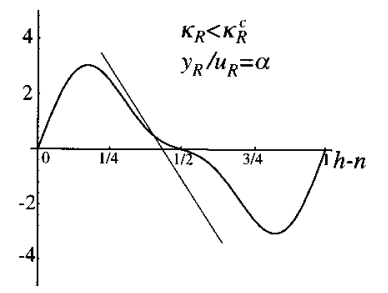

(b)

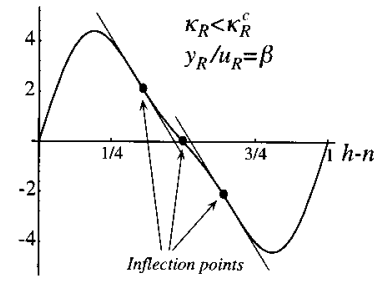

(c)

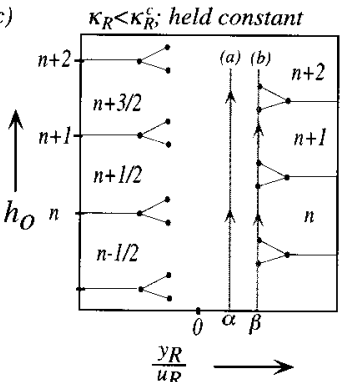

(d)

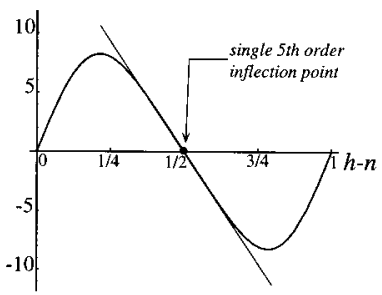

(e) $y_{R} / u_{R}=16$

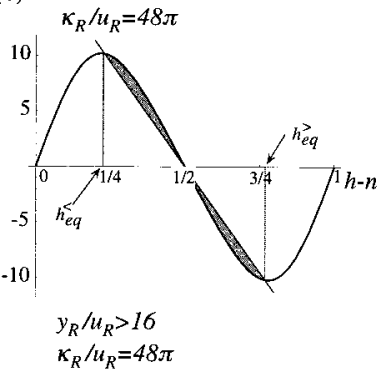

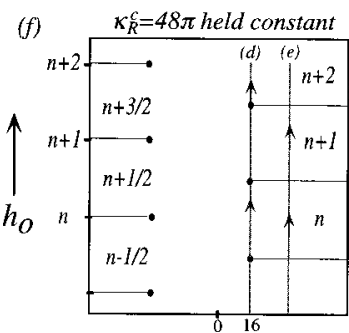

$\frac{y_{R}}{u_{R}} \longrightarrow$

FIG. 15. From triple point to tricriticality in the layering phase diagram for $u_{R}>0$. (a) Continuous increase in film thickness [shown as path $a$ in part (c) of this figure]. (b) Continuous increase in film thickness that just passes through the layering critical points [path $b$ in part (c)]. (c) Schematic phase diagram with substrate potential strength $\kappa_{R}<\kappa_{R}^{c}$ taken as fixed, independent of film thickness for simplicity. (d) Merging of the three inflection points into a single fifth-order inflection point at $\kappa_{R}=48 \pi$ and $y_{R} / u_{R}=16$. The triple point is now a tricritical point and film thickness grows continuously [path $d$ in part (f) of this figure]. (e) Ordinary first-order layering beyond the tricritical point [path $e$ in (f)]. (f) Schematic phase diagram for $\kappa_{R}=\kappa_{R}^{c}=48 \pi$.

We expect, then, that the stability of the first-order transition will continue to increase as $y_{R}$ increases. As further evidence for this we may examine the stability of the triple point, $y_{R}^{t}$. As shown in Fig. 15, the triple point becomes a tricritical point, and then a critical point when $\kappa_{R} / u_{R}$ becomes so large that the pair of inflection points on either side of $h=n+\frac{1}{2}$ merge with the one at $h=n+\frac{1}{2}$, forming a single fifth-degree inflection point (which, within Landau theory, defines a tricritical point). The vanishing of the third derivative at $h=n+\frac{1}{2}$ occurs when $y_{R} / u_{R}=16$. The slope at this point is then $k_{c}^{\mathrm{tri}}=12 \pi$. Thus only for $\kappa_{R} / u_{R}>3(4 \pi)^{2}$ is the triple point washed out. This is quite a bit larger than the value $(4 \pi)^{2}$ at which the first-order transition disappears at $y_{R}=0$.

We finally obtain, then, the following picture of the disappearance of the zipper with increasing $\kappa_{R}$ and/or decreasing $u_{R}$. The zipper, for a given value of $h_{0}$, first breaks in the middle $\left(y_{R}=0\right)$, forming a pair of two-pronged forks. The prongs then become shorter, eventually retracting into 

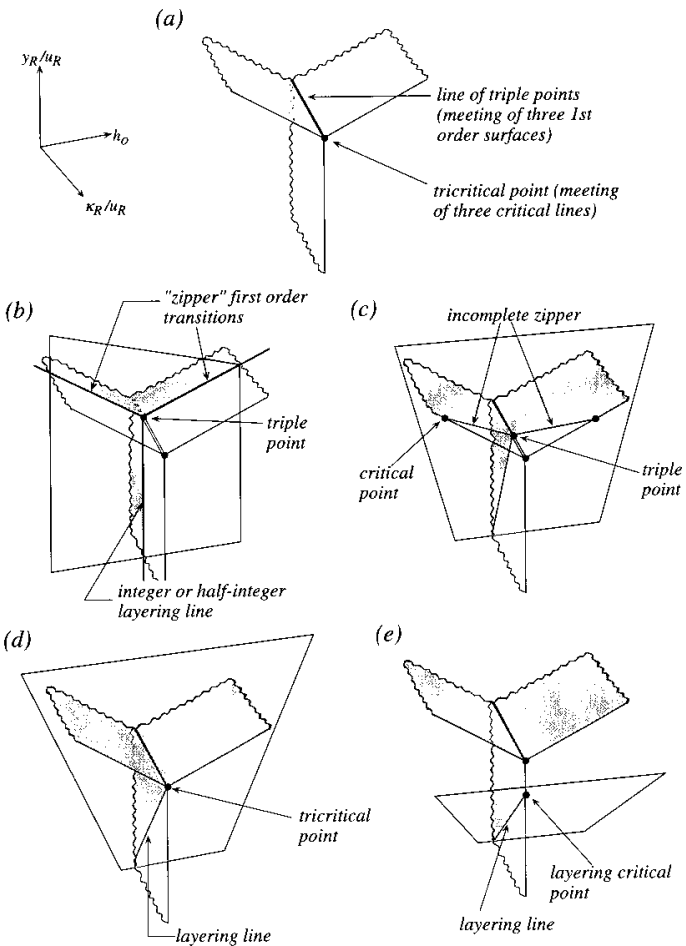

FIG. 16. Zippered layering phase diagram as a sequence of plane sections of the usual three-dimensional tricritical region.

the triple point. Precisely at the point where the prongs disappear, the triple point becomes a tricritical point. Beyond this the tricritical point becomes a simple Ising critical point, and locally the picture is now indistinguishable from Fig. 1(b). Now, $\kappa_{R}$ decreases as $h_{0}$ increases. Hence as long as $u_{R}>0$ remains fixed as the film thickens, this process occurs in reverse order, with the zipper reappearing for sufficiently thick films [see Fig. 1(c)]. In the higher, three-dimensional space, $\left(h_{0}, y_{R} / u_{R}, \kappa_{R} / u_{R}\right)$, this process can be viewed as a sequence of plane sections of the usual tricritical surface [see Fig. 16]. Consequently, as $K_{0}$ decreases toward the point at which $u$ becomes irrelevant, one has $u_{R} \rightarrow 0$, and ever thicker films will be required to see the zipper. This means that integer and half-integer layering transitions "unzip" from the bottom, becoming fully unzipped all the way to infinite layer thickness precisely when $u$ becomes marginal. ${ }^{22}$ Note that the mapping of the original model onto this fully renormalized description may lead to some nonmonotonic dependence of the renormalized parameters on the original ones (thus, for example, $u_{R}, y_{R}$, and $\kappa_{R}$ are all functions of $J_{1}$, $J_{2}$, and $T$ ). In thinner films one may, therefore, see behaviors different from the asymptotic behaviors we have found.

\section{2. $u_{R}<0$ : Spontaneously broken particle-hole symmetry and intermeshing}

We next consider the case $u_{R}<0$, which turns out to yield completely different behavior. The evolution of the substrate potential as $y_{R}$ goes through zero is shown in Fig. 17. This figure is actually identical to Fig. 13(a) turned upside down. The major difference now is that the absolute minima at integer $h=n$ split continuously in two at $y_{R}=4\left|u_{R}\right|$. The two new minima lie at $h=n \pm \theta\left(y_{R}\right)$ where $\theta\left(y_{R}\right)$ grows continuously from zero. Again, since this transition is continuous,

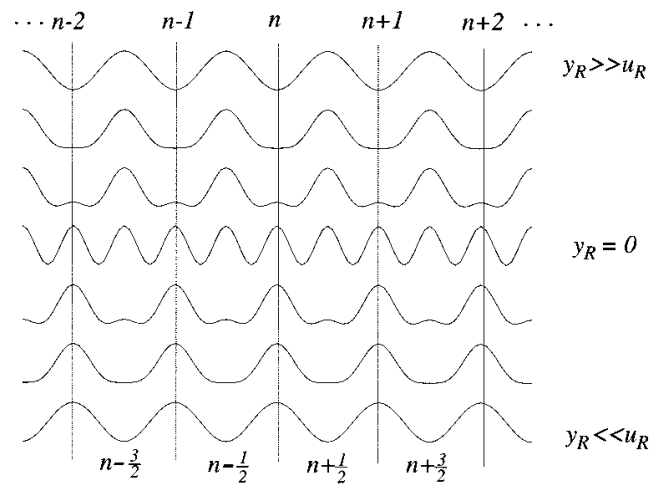

FIG. 17. Evolution of the bulk interface corrugation potential for $u_{R}<0$. The new $\theta$ DOF phase appears for an intermediate range of $y_{R}$.

fluctuation corrections will alter its nature and position. The transition, which appears as a classical Landau mean-field critical point in our theory, must become a two-dimensional Ising critical point with $\theta\left(y_{R}\right) \sim\left|y_{R}-y_{R}^{c}\right|^{1 / 8}$ and $y_{R}^{c} \leq 4\left|u_{R}\right|$. At $y_{R}=0$ one has, by symmetry, $\theta(0)=\frac{1}{4}$, so that the equilibrium mean surface heights are now $h=n \pm \frac{1}{4}$, rather than $h=n$ or $h=n+\frac{1}{2}$ as found when $u_{R}>0$. For $y_{R}<0$ the minima at $h=n+\theta\left(y_{R}\right)$ and $h=n+1-\theta\left(y_{R}\right)$ move together, eventually merging at $h=n+\frac{1}{2}$ when $y_{R}=-y_{R}^{c}$ $\gtrsim 4 u_{R}$. The merging also corresponds to a two-dimensional Ising critical point, with $\frac{1}{2}-\theta\left(y_{R}\right) \sim\left|y_{R}+y_{R}^{c}\right|^{1 / 8}$. For $y_{R}<$ $-y_{R}^{c}$ only minima at $h=n+\frac{1}{2}$ remain, signifying the usual DOF phase.

Thus instead of the preroughening line simply becoming first order, it splits into two second-order Ising lines, with a new intervening phase, which we call the $\theta$ DOF phase, ${ }^{4}$ with continuously varying mean surface height. Den $\mathrm{Nijs}^{4}$ first introduced this phase as a consequence of particle-hole symmetry violating terms in the Hamiltonian (which we neglect throughout this work), completely analogous to magneticfield terms in an Ising model. Here we find this phase as a result of spontaneous breaking of particle-hole symmetry driven by $u_{R}<0$.

The layering phase diagram is now very simple to describe. In the presence of the substrate potential it is clear by symmetry that neighboring minima can be degenerate only when $h_{0}=n$ or $h_{0}=n+\frac{1}{2}$. If $h_{0}=n+\frac{1}{2}$ degenerate minima are, for large positive $y_{R}, h \simeq n$ and $h \simeq n+1$, signifying the usual first-order transitions between essentially integer film thicknesses. However, when $y_{R} \lesssim y_{R}^{c}$ the degeneracy is between $h \simeq n+\theta\left(y_{R}\right)$ and $h \simeq n+1-\theta\left(y_{R}\right) \quad$ (approximate equality only due to the perturbative effect of $\kappa_{R}$ on the positions of the minima). When $y_{R} \geq-y_{R}^{c}$ these two minima merge, and the film thickness then varies continuously around $h=n+\frac{1}{2}$ for small deviations of $h_{0}$ from $n+\frac{1}{2}$. The layering line therefore terminates there in an Ising critical point. On the other hand, if $h_{0}=n$ degenerate minima are, for large negative $y_{R}, h \simeq n \pm \frac{1}{4}$. When $y_{R} \geq-y_{R}^{c}$ the degeneracy is between $h \simeq n \pm \theta\left(y_{R}\right)$, and when $y_{R} \leq y_{R}^{c}$ these two minima merge. The film thickness then varies continuously around $h=n$ for small deviations of $h_{0}$ from $n$. The layering line therefore again terminates in an Ising critical point, but this time as $y_{R}$ increases rather than decreases. In the inter- 


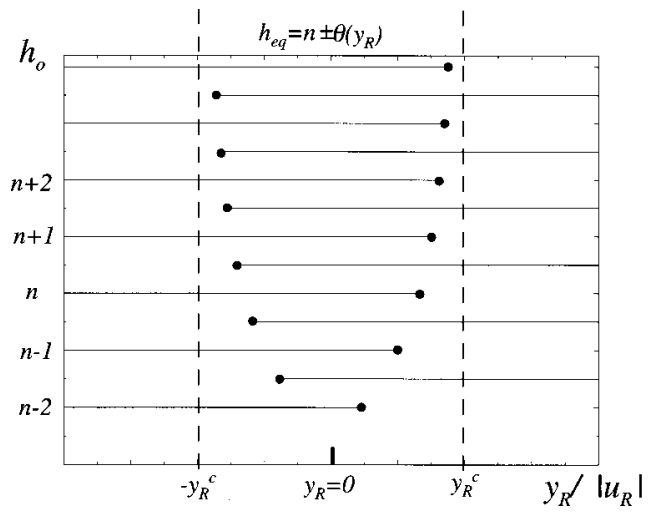

FIG. 18. Intermeshing layering phase diagram for $u_{R}<0$. The two sequences of layering critical points approach the boundaries of the $\theta \mathrm{DOF}$ phase as the film thickens.

mediate regime, $-y_{R}^{c} \lessgtr y_{R} \leq y_{R}^{c}$, both sets of lines exist. Thus, unlike the case $u_{R}=0$ where the two sets of lines are pushed apart so that there is a small region about $y_{R}=0$ where the film can grow continuously, the two sets, though independent and nonintersecting, are intermeshed so that at no time can one have unbounded continuous film growth [see Fig. 18; this figure is also reproduced, with more physical axes, in Fig. 1(d)]. Note, however, that if one varies $y_{R}$ in an oscillatory fashion as $h_{0}$ increases, one can, in principle, follow a snakelike path to grow an arbitrarily thick film without ever crossing a layering line. This is another signal that the bulk interface transition is second order rather than first order. As $n \rightarrow \infty$ the two sets of Ising critical points converge to the bulk interface Ising transitions at $y_{R}= \pm y_{R}^{c}$, with $T_{I, n}^{1}-T_{I}^{1}, T_{I}^{2}-T_{I, n}^{2} \sim n^{-(2+\alpha)}$, just as for first-order preroughening.

\section{G. Global phase diagram}

The computations in this section are relevant both to roughening and preroughening phenomena on the bulk interface and to layering phenomena in film growth. The results for $u_{R} \neq 0$ are new and, as we have seen, have strong impact on the phase diagrams. In particular, some previous results in the literature ${ }^{4}$ require some revision.

Thus, Fig. 2 in Ref. 4 shows the Ising transition between DOF and reconstructed phases joining the preroughening line precisely at the point labeled $N$ where $u$ becomes relevant. We believe this to be incorrect: the point $N$ will generically lie to the left (toward smaller $L=J_{2} / k_{B} T$ ) of the Ising line, which we expect, assuming that $u_{R}>0$ for the RSOS model, to join the first-order preroughening line at a critical end point $N^{\prime}$ distinct from $N$ [see the central phase diagram in Fig. 1]. The point $N$ is therefore tricritical, rather than bicritical as proposed by den Nijs. ${ }^{4}$ The physics of the Ising line is separate from that of the preroughening line (in fact, for a triangular lattice substrate the reconstructed phase, and hence the Ising line, is completely absent), and we see no reason why they should be connected at $N$. Our plaquette mean-field calculations will lend further credence to the distinction between $N$ and $N^{\prime}$. We shall find, however, that the first-order transition remains extremely weak, which may explain why it was not seen in earlier numerical investigations of the RSOS model.
Alternatively, if $u_{R}<0$ the preroughening line splits into two Ising lines at the point $N$, with the new $\theta$ DOF phase in between [see the inset to the central phase diagram in Fig. 1]. How the reconstructed-DOF (Ising) transition line connects up to this (if at all) is still not clear to us: this type of behavior is not seen in the RSOS models we investigate, but has been seen in the two-dimensional Ashkin-Teller model. ${ }^{23,24}$ As discussed in Appendix D, this model may be interpreted as a "binary alloy" interface model, but its detailed phase diagram lacks an obvious correspondence to that appearing in Fig. 1. Nevertheless, these results hint that the place to look for a $\theta$ DOF phase experimentally is in alloy films with more than one atomic (or molecular) constituent.

\section{CALCULATIONAL RESULTS}

\section{A. Single-spin computations}

Many of the phases we are interested in can be investigated, often analytically, within a simple single-spin meanfield formalism. In order to gain insight we begin with these computations. This allows one to discuss not only the usual layering phenomena, but also the interplay between the thinfilm analogues of reconstruction and roughening. In particular, we shall elucidate the nature of the reconstructed-rough phase. Multispin plaquettes will be used later to improve the accuracy of the calculations as well as to describe phases that the single-spin theory misses.

\section{Spin- $\frac{1}{2}$ computations: tricriticality and tetracriticality in reconstructed layering}

If, in addition, one is interested only in phenomena involving at most two different layers, a spin- $\frac{1}{2}$ model suffices. The Hamiltonian is given by Eq. (2.8), and its basic phenomenology was outlined in Sec. II B 1. Here we fill in some of the details via explicit computations.

Applying the formalism of Appendix A, the single site mean-field free-energy functional is

$$
\begin{aligned}
\mathcal{F}\left\{H_{i}, \sigma_{i} ; h_{i}\right\}= & -K \sum_{\langle i j\rangle} \sigma_{i} \sigma_{j}-L \sum_{(i k)} \sigma_{i} \sigma_{k}-\sum_{i}\left(H_{i}+h_{i}\right) \sigma_{i} \\
& -\sum_{i} \ln \cosh \left(H_{i}\right) .
\end{aligned}
$$

We assume that $L>0$, but that $K$ can have either sign. The first of the saddle-point equations (A9) yields $\sigma_{i}=$ $-\tanh \left(H_{i}\right)$. Substituting this relation into Eq. (4.1) we obtain the Bogoliubov mean-field free energy

$$
F\left\{h_{i}\right\}=\min _{\left\{\sigma_{i}\right\}}\left[A\left\{\sigma_{i}\right\}-\sum_{i} h_{i} \sigma_{i}\right],
$$

where the mean-field Helmholtz free energy is [see also Eq. (2.17) and the discussion preceding it]

$$
\begin{aligned}
A\left\{\sigma_{i}\right\}= & -K \sum_{\langle i j\rangle} \sigma_{i} \sigma_{j}-L \sum_{(i k)} \sigma_{i} \sigma_{k}-\frac{1}{2} \sum_{i}\left[\left(1-\sigma_{i}\right)\right. \\
& \left.\times \ln \left(1-\sigma_{i}\right)+\left(1+\sigma_{i}\right) \ln \left(1+\sigma_{i}\right)\right] .
\end{aligned}
$$

We restrict our attention to a bipartite lattice, with sublattices $A$ and $B$. Let there be $q_{1}$ nearest neighbors and $q_{2}$ next- 
nearest neighbors. We assume that the only relevant phases are those with uniform magnetization, $\sigma_{i}=m_{A}$ for $i \in A$ and $\sigma_{i}=m_{B}$ for $i \in B$, on each sublattice, with corresponding fields $h_{A}$ and $h_{B}$. The ferromagnetic and antiferromagnetic order parameters are, respectively, $m \equiv \frac{1}{2}\left(m_{A}+m_{B}\right)$ and $m^{\dagger}$ $\equiv \frac{1}{2}\left(m_{A}-m_{B}\right)$, with corresponding conjugate fields $h=h_{A}$ $+h_{B}$ and $h^{\dagger}=h_{A}-h_{B}$. In terms of these Eq. (4.2) becomes

$$
F\left\{h_{i}\right\} / N \equiv f\left(h, h^{\dagger}\right)=\min _{m, m^{\dagger}}\left[a\left(m, m^{\dagger}\right)-h m-h^{\dagger} m^{\dagger}\right],
$$

where the Helmholtz free energy per site is

$$
\begin{aligned}
a\left(m, m^{\dagger}\right) \equiv & A\left\{\sigma_{i}\right\} / N=-\frac{1}{2} c m^{2}-\frac{1}{2} c^{\dagger} m^{\dagger 2}+\frac{1}{4}\left(1-m-m^{\dagger}\right) \\
& \times \ln \left(1-m-m^{\dagger}\right)+\frac{1}{4}\left(1-m+m^{\dagger}\right) \ln \left(1-m+m^{\dagger}\right) \\
& +\frac{1}{4}\left(1+m-m^{\dagger}\right) \ln \left(1+m-m^{\dagger}\right)+\frac{1}{4}\left(1+m+m^{\dagger}\right) \\
& \times \ln \left(1+m+m^{\dagger}\right),
\end{aligned}
$$

and where $c=K q_{1}+L q_{2}$ and $c^{\dagger}=-K q_{1}+L q_{2}$. Notice that $c>c^{\dagger}$ whenever $K>0$ and $c^{\dagger}>c$ whenever $K<0$. When both $K$ and $L$ are positive we expect $m_{A}=m_{B}=m$ and $m^{\dagger}$ $=0$. In this case $a(m)$ is identical to the mean-field free energy of a model with nearest-neighbor interactions only, but effective coupling $K_{\text {eff }}=K+q_{2} L / q_{1}$, and we learn nothing new. The second-neighbor coupling gives rise to interesting new physics, then, only in the antiferromagnetic regime, $K<0$.

Let us then use Eq. (4.4) to understand the onset of antiferromagnetism. The antiferromagnetic-paramagnetic phase boundary is located by considering the stability of the paramagnetic phase, where $m^{\dagger}=0$, to nonzero $m^{\dagger}$. To this end, let $m_{0}(h)$ be the value of $m$ that minimizes the right-hand side of Eq. (4.4) with $m^{\dagger} \equiv 0$ and $h^{\dagger} \equiv 0$, i.e.,

$$
m_{0}(h)=\tanh \left[c m_{0}(h)+h\right],
$$

and let the corresponding free energy be $f_{0}(h)$. To see if this is the true minimum we Taylor expand the right-hand side of Eq. (4.4) in the deviations $\delta m \equiv m-m_{0}(h)$ and $m^{\dagger}$ (maintaining $\left.h^{\dagger}=0\right)$. Since we expect any phase transition to be driven by the onset of $m^{\dagger}$, we further minimize the resulting expression over $\delta m$ for a given $m^{\dagger}$, yielding

$$
\begin{aligned}
\delta m= & -\frac{m_{0}}{\left(1-m_{0}^{2}\right)\left[1-c\left(1-m_{0}^{2}\right)\right]} m^{\dagger 2} \\
& +\frac{m_{0}}{\left(1-m_{0}^{2}\right)^{3}\left[1-c\left(1-m_{0}^{2}\right)\right]}\left\{\frac{m_{0}^{2}}{\left[1-c\left(1-m_{0}^{2}\right)\right]^{2}}\right. \\
& \left.-\frac{1+3 m_{0}^{2}}{\left[1-c\left(1-m_{0}^{2}\right)\right]}+\left(1+m_{0}^{2}\right)\right\} m^{\dagger 4}+O\left(m^{\dagger 6}\right) .
\end{aligned}
$$

We obtain then the result

$$
\begin{aligned}
a\left(m, m^{\dagger}\right)-h m= & f_{0}\left(m_{0}\right)+\frac{1}{2} r^{\dagger} m^{\dagger 2}+u^{\dagger} m^{\dagger 4}+v^{\dagger} m^{\dagger 6}+w^{\dagger} m^{\dagger 8} \\
& +O\left(m^{\dagger 10}\right),
\end{aligned}
$$

where

$$
\begin{gathered}
r^{\dagger}=\frac{1-c^{\dagger}\left(1-m_{0}^{2}\right)}{\left(1-m_{0}^{2}\right)}, \\
u^{\dagger}=\frac{\left(1-3 m_{0}^{2}\right)-c\left(1+3 m_{0}^{2}\right)\left(1-m_{0}^{2}\right)}{12\left(1-m_{0}^{2}\right)^{3}\left[1-c\left(1-m_{0}^{2}\right)\right]}, \\
v^{\dagger}=\frac{m_{0}^{2}}{6\left(1-m_{0}^{2}\right)^{5}\left[1-c\left(1-m_{0}^{2}\right)\right]^{3}}\left[\left(m_{0}^{2}-3\right)+3 c\left(m_{0}^{2}+3\right)\right. \\
\left.\times\left(1-m_{0}^{2}\right)-6 c^{2}\left(1+m_{0}^{2}\right)\left(1-m_{0}^{2}\right)^{2}\right] .
\end{gathered}
$$

We have therefore obtained a standard Landau free-energy functional for $m^{\dagger}$. Thus, if $u^{\dagger}>0$, there is an instability toward antiferromagnetism when $r^{\dagger}<0$. The antiferromagnetic critical point therefore occurs when

$$
c^{\dagger} \equiv T^{\dagger}(h) / T_{0}^{\dagger}=1-m_{0}(h)^{2},
$$

where $T_{0}^{\dagger}=\left(\left|J_{1}\right| q_{1}+J_{2} q_{2}\right) / k_{B} \equiv J_{0}^{\dagger} / k_{B}$ is the transition temperature at $h=0$. Note that $T / T_{0}^{\dagger}<1$. For very large $h$, $m_{0}(h)$ will be very close to unity, and $r^{\dagger}>0$. As $h$ decreases, for a given fixed $T<T_{0}^{\dagger}, m_{0}(h)$ decreases and eventually the phase boundary will be reached for some critical $h=h^{\dagger}(T)$, which increases as $T$ decreases. Below the transition $m^{\dagger}$ increases continuously from zero as $m^{\dagger} \sim\left(h^{\dagger}-h\right)^{\beta}$ with $\beta=\frac{1}{2}$ in this mean-field approximation (an exact theory would yield the two-dimensional Ising result $\beta=\frac{1}{8}$ ).

Another possibility is that $u^{\dagger}<0$ but $v^{\dagger}>0$. In this case the transition will be first order, with the minimum at $m^{\dagger}$ $=0$ trading stability with two degenerate minima at nonzero $m^{\dagger}$. The point $u^{\dagger}=r^{\dagger}=0$ where the transition converts from second to first order is then a tricritical point. From Eq. (4.9) we see that $u^{\dagger}$ is positive for

$$
c \equiv T / T_{0}>\frac{\left(1-m_{0}^{2}\right)\left(1+3 m_{0}^{2}\right)}{1-3 m_{0}^{2}},
$$

where $T_{0}=\left(q_{2} J_{2}-q_{1}\left|J_{1}\right|\right) / k_{B} \equiv J_{0} / k_{B}$. This inequality will be valid for sufficiently small $m_{0}$. Therefore the antiferromagnetic transition line will be second order if $T / T_{0}^{\dagger}$ is large enough. On the other hand, the simultaneous condition $r^{\dagger}$ $=0$ and $u^{\dagger}=0$ then yields a tricritical point $T_{\text {tri }}$ at

$$
\frac{k_{B} T_{\text {tri }}}{J_{0}^{\dagger}}=\frac{2}{3} \frac{1+2 J_{0} / J_{0}^{\dagger}}{1+J_{0} / J_{0}^{\dagger}}
$$

This actually leads to a line of tricritical points in the $K-L$ plane. It is easy to check that $u^{\dagger}$ remains negative for all $T$ $<T_{\text {tri }}$.

When $T<T_{\text {tri }}$ the transition line is no longer given by $r^{\dagger}$ $=0$. Rather, one must look to see when the minimum at $m^{\dagger}=0$ is no longer the absolute minimum. For small negative $u^{\dagger}$ one then finds a first-order transition at $r^{\dagger}=u^{\dagger 2} / 2 v^{\dagger}$ $>0$ at which $m^{\dagger}$ jumps from zero to $m^{\dagger}=\sqrt{-u^{\dagger} / 2 v^{\dagger}}$. The transition therefore takes place before the putative secondorder line at $r^{\dagger}=0$.

The tricritical point exists so long as $v^{\dagger}>0$. One may check the sign of $v^{\dagger}$ on the tricritical line and verify that it is positive for 
(i)

(ii)

(iii)

(iv)

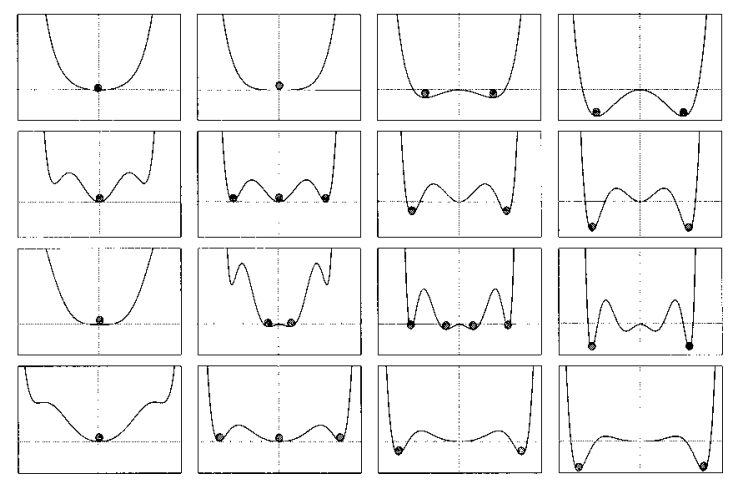

FIG. 19. Behavior of the free energy in the vicinity of the tetracritical point. The sequences shown correspond to paths $(i),(i i)$, (iii), and (iv) in Fig. 5(a).

$$
\frac{k_{B} T}{J_{0}^{\dagger}}>\frac{11-\sqrt{33}}{6} \simeq 0.8759 .
$$

The point $k_{B} T_{\text {tet }} / J_{0}^{\dagger}=(11-\sqrt{33}) / 6$ is tetracritical since $r^{\dagger}, \quad u^{\dagger}$, and $v^{\dagger}$ all vanish simultaneously. For $k_{B} T / J_{0}^{\dagger}$ $<(11-\sqrt{33}) / 6$ new behavior occurs. In Fig. 19 we show the structure of the minima in the free energy, Eq. (4.8). One finds that the tricritical point now becomes a critical end point that terminates the second-order line before the firstorder line ends. The first-order line now terminates in a critical point completely within the antiferromagnetic phase (see Fig. 5). Thus at temperatures below the critical end point, as a function of magnetic field, there is a first-order transition to the antiferromagnetic phase, while above it the transition is second order. However, at temperatures above, but close to the critical end point, the second-order transition is followed by a first-order transition from one nonzero value of $m^{\dagger}$ to another. A 3D view of this structure is shown in Fig. 5(b). Note that the antiferromagnetic transition in the model with nearest-neighbor interactions only $\left(J_{2}=0\right)$ is always second order.

\section{Spin-1 computations: the reconstructed-rough phase}

We consider next the spin-1 model (2.9). This will allow us to deal with phases and phase transitions involving three different layer thicknesses. It will turn out that this model contains essentially all the physics needed to explain all the phases in the exact Hamiltonian. The simplest application is to layering in the ferromagnetic regime where the spin-1 model exhibits two layering transitions at low temperatures: between the phase with $m \simeq-1$ and the phase with $m \simeq 0$ and between the phase with $m \simeq 0$ and the phase with $m$ $\simeq 1$. However, the results here do not contain any new physics, and the extension to the full layering phase diagram, Fig. 1(a), is clear. In this subsection, therefore, we focus instead on the film analogue of the reconstructed-rough phase, and transitions from it to the reconstructed-flat and disordered flat phases. All of these may be elucidated from the singlesite mean-field theory. Only the preroughening transition between the flat and disordered flat phases requires the retention of a plaquette of spins, and this will be discussed in Sec. IV C.

For the spin- $-\frac{1}{2}$ model the RSOS condition was redundant because the spins could take only two values. In the spin-1 case we must include it explicitly. Since we are still dealing only with single-site mean-field theories the condition must be imposed on the mean field alone and the discussion in Sec. III C 2 is relevant. We choose to approximate the RSOS condition by a nearest-neighbor quartic interaction. Thus we consider the mean-field theory of the Hamiltonian,

$$
\begin{aligned}
\overline{\mathcal{H}}_{1}^{\prime}= & \overline{\mathcal{H}}_{1}+\frac{1}{4} K^{\prime} \sum_{\langle i j\rangle}\left(s_{i}-s_{j}\right)^{4}=-\left(K+2 K^{\prime}\right) \sum_{\langle i j\rangle} s_{i} s_{j} \\
& +\frac{3}{2} \sum_{\langle i j\rangle} s_{i}^{2} s_{j}^{2}-L \sum_{(i k)} s_{i} s_{k}-h \sum_{i} s_{i}+\widetilde{h_{2}} \sum_{i} s_{i}^{2},
\end{aligned}
$$

with $K^{\prime} \equiv J_{1}^{\prime} / k_{B} T>0, \quad \widetilde{h_{2}}=h_{2}+\frac{1}{2} K q_{1}+\frac{1}{4} K^{\prime} q_{1}+\frac{1}{2} L q_{2}$ and we have used the fact that $s_{i}^{3}=s_{i}$.

Since the interactions between spins now include quadratic terms the formalism in Appendix A tells us that the most general single-site free-energy functional we need to consider is

$$
\begin{aligned}
\Phi\left(H, H_{2}\right) & =-\ln \left(\frac{1}{3} \sum_{s=0, \pm 1} e^{-H s-H_{2} s^{s^{2}}}\right) \\
& =-\ln \left\{\frac{1}{3}\left[1+2 e^{-H_{2}} \cosh (H)\right]\right\} .
\end{aligned}
$$

The full mean-field free-energy functional is now obtained by associating independent saddle-point theory variables $\sigma_{i}$ with $s_{i}$ and $\tau_{i}$ with $s_{i}^{2}$, yielding

$$
\begin{aligned}
\mathcal{F}= & -\left(K+2 K^{\prime}\right) \sum_{\langle i j\rangle} \sigma_{i} \sigma_{j}+\frac{3}{2} K^{\prime} \sum_{\langle i j\rangle} \tau_{i} \tau_{j}-L \sum_{(i k)} \sigma_{i} \sigma_{k} \\
& -\sum_{i}\left(H_{i}+h\right) \sigma_{i}-\sum_{i}\left(H_{2, i}-\widetilde{h}_{2}\right) \tau_{i}+\sum_{i} \Phi\left(H_{i}, H_{2, i}\right) .
\end{aligned}
$$

The only coupling between the $\sigma$ 's and the $\tau$ 's is indirectly through the coupling of the $H$ 's and $H_{2}$ 's in $\Phi$. Notice the antiferromagnetic coupling between the $\tau$ 's.

To elucidate the nature of the reconstructed-rough phase, specialize to $h=0$. What we will show is that when $J_{1}<0$ and $J_{2}$ is not too large there exists a phase in which $H_{i}, \sigma_{i}$ $\equiv 0$, but $\tau_{i}$ has long-range antiferromagnetic order. This means that the magnetization vanishes on all sites, but there is antiferromagnetic order in the magnitude of the fluctuations on each site. This is intuitively plausible because if we consider the special case $J_{2}=0$, the exact $T=0$ ground state of $\mathcal{H}_{1}^{\prime}$ has $s_{i} \equiv 0$ on one sublattice and $s_{i}= \pm 1$ randomly on the other sublattice. Thus even though $\left\langle s_{i}\right\rangle=0$ everywhere, $s_{i}^{2}$ alternates between 0 and 1 . Note that the RSOS condition is required to stabilize this state: in its absence the ground state would have $s_{i}=1$ on one sublattice and $s_{i}=-1$ on the other. Similarly, in our mean-field treatment we expect such a state to exist only in a certain range of sufficiently large $J_{1}^{\prime}$. At high enough temperature we expect this order to be destroyed, signaling the film analogue of the reconstructed rough to fully rough transition [the negative part of the $K$ axis in the central phase diagram of Fig. 1]. We shall also see below that inclusion of $J_{2}>0$ allows for a transition to a phase with true antiferromagnetic order in the $s_{i}$. This cor- 
responds to the film analogue of the reconstructed-rough to reconstructed-flat transition [path 5 in Fig. 1]. This is again intuitively plausible because $J_{2}>0$ will force the ground state to break the symmetry of $s_{i}= \pm 1$ on the second sublattice, forcing all these $s_{i}$ to take a common value. There will then be a first-order layering-type transition as a function of field, $h$, between the state with alternating 0 's and 1's and that with alternating 0 's and -1 's. When $J_{2}$ is large enough we shall find that the antiferromagnetic order can be lost with increasing temperature before the layering line terminates, corresponding to a reconstructed-flat to disordered flat transition [path 4 in Fig. 1]. Thus long-range positional order in the 0 's and 1's (or 0's and -1 's) can be lost while still maintaining a broken symmetry between 1 's and -1 's. This is the film analogue of the $K<0$ region of the DOF phase. As mentioned earlier, to describe the film analogue of the preroughening transition in the $K>0$ region of the central phase diagram in Fig. 1 we will require a plaquette of more than one spin (see Sec. IV C below).

Specializing the free-energy functional (4.16) to a two sublattice stucture for $\sigma_{i}, \tau_{i}$, and $H_{2, i}$ we have

$$
\begin{aligned}
f \equiv & \mathcal{F} / N=-\frac{1}{2}\left(K+2 K^{\prime}\right) q_{1} m_{A} m_{B}-\frac{1}{4} L q_{2}\left(m_{A}^{2}+m_{B}^{2}\right) \\
& -\frac{1}{2}\left(H_{A}+h\right) m_{A}-\frac{1}{2}\left(H_{B}+h\right) m_{B}+\frac{3}{4} K^{\prime} q_{1} \tau_{A} \tau_{B} \\
& -\frac{1}{2}\left(H_{2 A}-\widetilde{h_{2}}\right) \tau_{A}-\frac{1}{2}\left(H_{2 B}-\widetilde{h_{2}}\right) \tau_{B}-\frac{1}{2} \ln \left\{\frac { 1 } { 3 } \left[1+2 e^{-H_{2 A}}\right.\right. \\
& \left.\left.\times \cosh \left(H_{A}\right)\right]\right\}-\frac{1}{2} \ln \left\{\frac{1}{3}\left[1+2 e^{-H_{2 B}} \cosh \left(H_{B}\right)\right]\right\} .
\end{aligned}
$$

Variation with respect to $H_{2 A}, H_{2 B}$ yields

$$
\tau_{A}=\frac{2 e^{-H_{2 A}} \cosh \left(H_{A}\right)}{1+2 e^{-H_{2 A}} \cosh \left(H_{A}\right)}, \quad \tau_{B}=\frac{2 e^{-H_{2 B}} \cosh \left(H_{B}\right)}{1+2 e^{-H_{2 B}} \cosh \left(H_{B}\right)} .
$$

Variation with respect to $H_{A}$ and $H_{B}$ yields

$$
\begin{aligned}
& m_{A}=-\frac{2 e^{-H_{2 A} \sinh \left(H_{A}\right)}}{1+2 e^{-H_{2 A}} \cosh \left(H_{A}\right)}, \\
& m_{B}=-\frac{2 e^{-H_{2 B} \sinh \left(H_{B}\right)}}{1+2 e^{-H_{2 B}} \cosh \left(H_{B}\right)} .
\end{aligned}
$$

Inverting these and substituting them back into (4.17) we obtain the Bogoliubov free energy,

$$
\begin{aligned}
f_{\text {Bog }}= & -\frac{1}{2}\left(K+2 K^{\prime}\right) q_{1} m_{A} m_{B}-\frac{1}{4} L q_{2}\left(m_{A}^{2}+m_{B}^{2}\right)-\frac{1}{2} h\left(m_{A}\right. \\
& \left.+m_{B}\right)+\frac{3}{4} K^{\prime} q_{1} \tau_{A} \tau_{B}+\frac{1}{2}\left(\tau_{A}+\tau_{B}\right)\left[\widetilde{h}_{2}-\ln (2)\right]+\ln (3) \\
& +\frac{1}{2}\left(1-\tau_{A}\right) \ln \left(1-\tau_{A}\right)+\frac{1}{2}\left(1-\tau_{B}\right) \ln \left(1-\tau_{B}\right)+\frac{1}{4}\left(\tau_{A}\right. \\
& \left.+m_{A}\right) \ln \left(\tau_{A}+m_{A}\right)+\frac{1}{4}\left(\tau_{A}-m_{A}\right) \ln \left(\tau_{A}-m_{A}\right)+\frac{1}{4}\left(\tau_{B}\right. \\
& \left.+m_{B}\right) \ln \left(\tau_{B}+m_{B}\right)+\frac{1}{4}\left(\tau_{B}-m_{B}\right) \ln \left(\tau_{B}-m_{B}\right) .
\end{aligned}
$$

Focusing first on the reconstructed-rough phase, we set $h=0$ and assume that $m_{A}=m_{B}=0$. Minimizing $f_{\mathrm{Bog}}$ with respect to $\tau_{A}$ and $\tau_{B}$ then yields

$$
\tau_{A}=\frac{2 e^{-3 / 2 K^{\prime} q_{1}\left(\tau_{B}-\tau_{0}\right)}}{1+2 e^{-3 / 2 K^{\prime} q_{1}\left(\tau_{B}-\tau_{0}\right)}}, \quad \tau_{B}=\frac{2 e^{-3 / 2 K^{\prime} q_{1}\left(\tau_{A}-\tau_{0}\right)}}{1+2 e^{-3 / 2 K^{\prime} q_{1}\left(\tau_{A}-\tau_{0}\right)}},
$$

where $\tau_{0}=-\frac{2}{3} \widetilde{h_{2}} / K^{\prime} q_{1}$ and $\widetilde{h_{2}}$ was defined below Eq. (4.14). At high temperatures we expect $\tau_{A}=\tau_{B} \equiv \bar{\tau}$ with 0 $\leqslant \bar{\tau} \leqslant 1$ satisfying

$$
\bar{\tau}=\frac{2 e^{-3 / 2 K^{\prime} q_{1}\left(\bar{\tau}-\tau_{0}\right)}}{1+2 e^{-3 / 2 K^{\prime} q_{1}\left(\bar{\tau}-\tau_{0}\right)}} .
$$

As $T$ decreases we expect an instability either to a state with $\tau^{\dagger} \equiv \frac{1}{2}\left(\tau_{A}-\tau_{B}\right) \neq 0$, but $m_{A}=m_{B}=0$ still, or to a state with $m_{A}=m_{B} \equiv m \neq 0$, but $\tau^{\dagger}=0$. Treating the first case first, let $\tau=\frac{1}{2}\left(\tau_{A}+\tau_{B}\right)$ and $\delta \tau=\tau-\bar{\tau}$. Completely analogous to the computation leading to Eq. (4.8), we expand the free energy in a double Taylor series in $\tau^{\dagger}$ and $\delta \tau$. Mininimizing the result over $\delta \tau$ we find

$$
\delta \tau=-\frac{1}{2} \frac{(1-\bar{\tau})^{-2}-\bar{\tau}^{-2}}{3 K^{\prime} q_{1} / 2+\bar{\tau}^{-1}(1-\bar{\tau})^{-1}} \tau^{\dagger 2}+O\left(\tau^{\dagger 4}\right) .
$$

Substituting this into the free energy we obtain the Landau expansion in $\tau^{\dagger}$ alone:

$$
f_{\mathrm{Bog}}=f_{0}+\frac{1}{2} r^{\dagger} \tau^{\dagger 2}+u^{\dagger} \tau^{\dagger 4}+O\left(\tau^{\dagger 6}\right)
$$

with

$$
\begin{gathered}
f_{0}=\frac{3}{4} K^{\prime} q_{1} \bar{\tau}^{2}=\ln (3)+\left[\widetilde{h}_{2}-\ln (2)\right] \bar{\tau}+\bar{\tau} \ln (\bar{\tau}) \\
+(1-\bar{\tau}) \ln (1-\bar{\tau}), \\
r^{\dagger}=\bar{\tau}^{-1}(1-\bar{\tau})^{-1}-\frac{3}{2} K^{\prime} q_{1}, \\
u^{\dagger}=\frac{1}{12}\left[(1-\bar{\tau})^{-3}+\bar{\tau}^{-3}\right] \\
-\frac{1}{8} \frac{\left[(1-\bar{\tau})^{-2}-\bar{\tau}^{-2}\right]^{2}}{3 K^{\prime} q_{1} / 2+\bar{\tau}^{-1}(1-\bar{\tau})^{-1}} .
\end{gathered}
$$

There is a unique minimum at $\tau^{\dagger}=0$ for $\bar{\tau}^{-1}(1-\bar{\tau})^{-1}$ $=3 K^{\prime} q_{1} / 2$. Therefore the critical point occurs at a temperature $T_{c}$ determined by

$$
\bar{\tau}_{c}\left(1-\bar{\tau}_{c}\right)=\bar{T}_{c}
$$

where $\bar{T} \equiv 2 k_{B} T / 3 J_{1}^{\prime} q_{1}$ and

$$
\bar{\tau}_{c}=\frac{2 e^{-\left(\bar{\tau}_{c}-\tau_{0}\right) / \bar{T}_{c}}}{1+2 e^{-\left(\bar{\tau}_{c}-\tau_{0}\right) / \bar{T}_{c}}} .
$$

It is easy to check that $u^{\dagger}>0$ at this point. Since $\bar{\tau}(1-\bar{\tau})$ $\leqslant \frac{1}{4}$ it is clear that $\tau^{\dagger}=0$ for $\bar{T}>\frac{1}{4}$. Whether or not solutions to Eqs. (4.25),(4.26) exist depends on the temperatureindependent parameter $\tau_{0}=-\left(2 H_{2}+J_{1} q_{1}+J_{2} q_{2}\right.$ $\left.+J_{1}^{\prime} q_{1} / 2\right) / 3 J_{1}^{\prime} q_{1}$ (recall that $H_{2}=k_{B} T h_{2}$ is the curvature of the substrate potential). For example, if $\tau_{0}=\frac{2}{3}$ then $\bar{\tau}=\frac{2}{3}$ for all $\bar{T}$, and we have $\bar{T}_{c}=\frac{2}{9}$. If $H_{2}=0$ on a square lattice $\left(q_{1}\right.$ $=4$ ) then this situation corresponds to $\left|J_{1}\right| / J_{1}^{\prime}=\frac{3}{2}$. The maxi- 
mal $\bar{T}_{c}$ is $\frac{1}{4}$ and corresponds to $\bar{\tau}_{c}=\frac{1}{2}$. This occurs for $\tau_{0}$ $=(2-\ln (2)) / 4 \simeq 0.3267$. The general solution for $\tau_{0}$ given any $0<\bar{\tau}_{c}<1$ is

$$
\begin{aligned}
\tau_{0} & =\bar{\tau}_{c}\left(1-\bar{\tau}_{c}\right)\left\{\left(1-\bar{\tau}_{c}\right)^{-1}+\ln \left[\bar{\tau}_{c} / 2\left(1-\bar{\tau}_{c}\right)\right]\right\} \\
& \approx\left\{\begin{array}{l}
\bar{\tau}_{c} \ln \left(\bar{\tau}_{c} / 2\right) \rightarrow 0, \quad \bar{\tau}_{c} \rightarrow 0 \\
1-\left(1-\bar{\tau}_{c}\right) \ln \left[2\left(1-\bar{\tau}_{c}\right)\right] \rightarrow 1, \quad \bar{\tau}_{c} \rightarrow 1
\end{array}\right.
\end{aligned}
$$

Treating now the second case, we take $\tau^{\dagger}=0$ and expand the free energy in $\delta \tau$ and $m$. Again, minimizing the result with respect to $\delta \tau$ for given $m$ we obtain

$$
\delta \tau=\frac{1-\bar{\tau}}{3 \bar{\tau}} m^{2}+O\left(m^{4}\right) .
$$

Substituting this back into the free energy we obtain a Landau expansion in $m$ alone:

$$
f_{\mathrm{Bog}}=f_{0}+\frac{1}{2} r m^{2}+u m^{4}+O\left(m^{6}\right),
$$

with

$$
\begin{gathered}
r=\bar{\tau}^{-1}-\left(K+2 K^{\prime}\right) q_{1}-L q_{2} \\
u=\left(2 \bar{\tau}^{-2}+\bar{\tau}^{-3}\right) / 36>0 .
\end{gathered}
$$

There is a phase transition to a ferromagnetic phase at $r$ $=0$. This yields a critical temperature $T_{0}$ determined by

$$
\bar{\tau}_{0} \equiv \bar{\tau}\left(T_{0}\right)=\widetilde{T}_{0}, \quad \widetilde{T} \equiv k_{B} T /\left(J_{1} q_{1}+2 J_{1}^{\prime} q_{1}+J_{2} q_{2}\right) \text {. }
$$

The temperatures $T_{c}$ and $T_{0}$ coincide when $J_{1}$, $J_{1}^{\prime}, J_{2}$, and $h_{2}$ satisfy the constraint

$$
\begin{gathered}
\tau_{0}=\rho(1-\rho)\left\{\rho^{-1}+\ln [(1-\rho) / 2 \rho]\right\}, \\
\rho \equiv\left(J_{1} q_{1}+2 J_{1}^{\prime} q_{1}+J_{2} q_{2}\right) /\left(3 J_{1}^{\prime} q_{1} / 2\right),
\end{gathered}
$$

where $\tau_{0}$ was defined below Eq. (4.27). For given $J_{1}, J_{1}^{\prime}$ and $h_{2}$ it is easy to check that for $J_{2}$ larger than that satisfying Eq. (4.33) one has $T_{0}>T_{c}$ : the instability to the thin-film analogue of the DOF phase occurs first, with a transition to a reconstructed checkerboard state occurring only at lower temperature. Conversely, for smaller $J_{2}$ one has $T_{c}>T_{0}$ : the transition to the film analogue of the reconstructed-rough phase occurs first, with the transition to the true antiferromagnetic state occuring only at lower temperature. The special value of $J_{2}$ at which $T_{0}=T_{c}$ is bicritical with a direct transition from the paramagnetic to antiferromagnetic state. In Fig. 20 we show a numerical computation of the full phase diagram in the $H-T$ plane for various values of $J_{2}$. The behavior of the phase boundary near $T_{c}$ changes as $T_{0}$ passes through $T_{c}$ : one finds that $T_{\operatorname{Rec}}(H)-T_{c} \sim H^{2}$ for $T_{c}$ $>T_{0} ; \quad T_{\operatorname{Rec}}(H)-T_{c} \sim H^{2 / 3}$ for $T_{c}=T_{0} ;$ and $T_{\operatorname{Rec}}(H)-T_{c}$ $\sim|H|$ for $T_{c}<T_{0}$.

\section{B. Plaquette computations and preroughening}

We have now established all of the essential physics of the various phases in the antiferromagnetic regime where we have seen that a single-spin mean-field theory suffices. We (a)

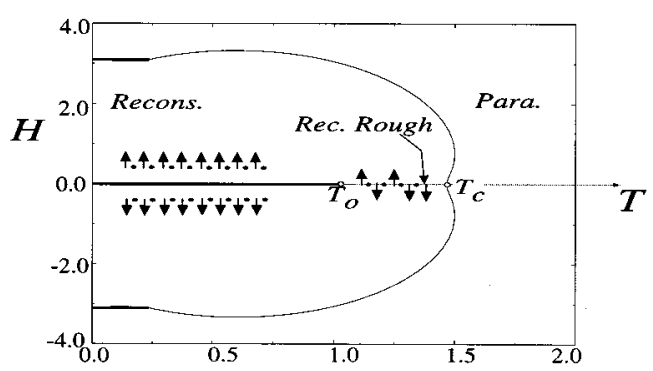

(b)

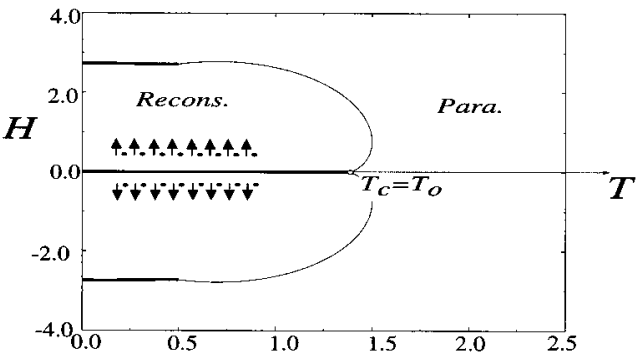

(c)

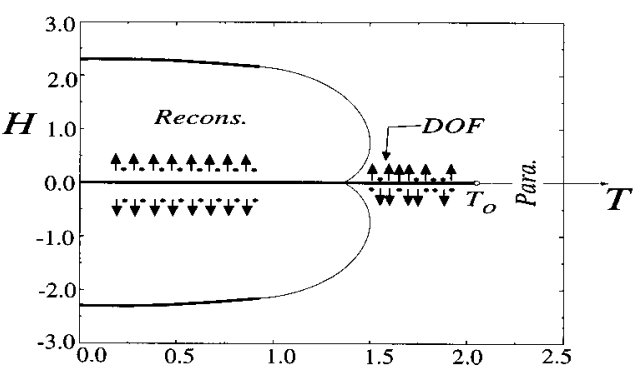

FIG. 20. Spin-1 phase diagrams showing the thin-film analogues of the reconstructed flat, reconstructed-rough and disordered flat phases as the second-neighbor coupling, $J_{2}$, varies: (a) small $J_{2}$, showing the termination of the antiferromagnetic layering line inside the reconstructed-rough phase; (b) bicritical value of $J_{2}$, showing a direct transition from the rough to antiferromagnetic phase; (c) large $J_{2}$, showing first the appearance of the DOF phase, followed by the antiferromagnetic phase.

now turn to the more experimentally relevant problem of understanding the thin-film analogue of the actual preroughening transition when $J_{1}>0$ (where it is now safe to take $\left.J_{1}^{\prime} \equiv 0\right)$. In this subsection we carry out detailed calculations using the plaquette mean-field formalism constructed in Sec. II. We begin with the simplest spin-1 model on the square lattice, Eq. (2.13), and the two sublattice magnetizations, Eq. (2.12). The function $\Phi^{(4)}\left(H_{A}, H_{B}\right)$ is computed in Appendix C.

As we have discussed, the film analogue of preroughening corresponds to a second-order phase transition at $h=0$ to a state with uniform magnetization $M_{A}=M_{B} \equiv M \neq 0$. As usual, we perform a Taylor expansion of the free energy in $M$ in the neighborhood of the transition. From Eq. (2.15) we have $-H=\gamma_{4} M+h$, where $\gamma_{4} \equiv 2 \lambda_{1} K+3 \lambda_{2} L$. Therefore $H$ will be small when $M$ is.

To proceed correctly, we first solve Eq. (2.14), which now reads

$$
M=\frac{1}{4} \frac{\partial \Phi^{(4)}}{\partial H},
$$


for $H(M)$. Using Eq. (C4) and defining

$$
\left\langle l^{p}\right\rangle \equiv\left(\sum_{l=0}^{4} l^{p} a_{l}\right) /\left(\sum_{l=0}^{4} a_{l}\right)
$$

we then obtain

$$
-H=\frac{4}{\left\langle l^{2}\right\rangle}+\frac{32}{3} \frac{\left\langle l^{4}\right\rangle-3\left\langle l^{2}\right\rangle}{\left\langle l^{2}\right\rangle^{4}} M^{3}+O\left(M^{5}\right) .
$$

Substituting this back into $f^{(4)}$ and using Eq. (2.17) we obtain the Landau expansion for the Helmholtz free energy,

$$
\frac{1}{N} A^{(4)}=f_{0}^{(4)}+\frac{1}{2} \chi^{-1} M^{2}+u M^{4}+O\left(M^{4}\right),
$$

where

$$
\chi^{-1}=\left(\frac{\partial^{2} A^{(4)}}{\partial M^{2}}\right)_{M=0}=\frac{4}{\left\langle l^{2}\right\rangle}-\gamma_{4}
$$

is the zero-field inverse susceptibility in the paramagnetic phase, and

$$
f_{0}^{(4)}=-\frac{1}{4} \ln \left(\sum_{l=0}^{p} a_{l}\right), \quad u=\frac{8}{3} \frac{3\left\langle l^{2}\right\rangle^{2}-\left\langle l^{4}\right\rangle}{\left\langle l^{2}\right\rangle^{4}}
$$

As usual, if $u$ is positive (which may be checked), the second-order phase transition takes place in zero field when the susceptibility diverges, i.e., $\chi^{-1}=0$. We must therefore solve the equation

$$
\frac{4}{\gamma_{4}}=\left(\sum_{l=0}^{p} l^{2} a_{l}\right) /\left(\sum_{l=0}^{p} a_{l}\right),
$$

which will yield a critical surface in the three parameter space $\left(K, L, h_{2}\right)$.

Let us first examine limiting cases. At low temperatures where $K$ and $L$ are large (and positive) the right-hand side of Eq. (4.40) vanishes exponentially in $1 / T$ since $x, y$, and $z$ (defined in Appendix C) do. The inverse of this term therefore dominates at low temperature, $\chi^{-1}$ is large and positive, $M=0$, and there can be no symmetry breaking. Similarly, at high temperature the inverse of this term again dominates since it remains of order unity while $\gamma_{4}$ vanishes as $1 / T$. Thus $\chi^{-1}$ is again positive and $M=0$. We have therefore established the reentrant property of the phase transition, assuming that it exists.

To establish existence, let us look at the simplest possible case, $K=h_{2}=0$, keeping only $L>0$ (note that $\widetilde{h}_{2}$ is positive even if $h_{2}=0$, which reflects the fact that fluctuations renor- malize $h_{2}$ to larger values because there is more fluctuation entropy available when the spin is zero). We then have $y$ $=1$ and $z=e^{-\gamma_{4} / 2}$. Defining $\bar{x} \equiv x^{2}=e^{-L}$ and taking $\lambda_{2}$ $=1$ so that $z=x^{3}$, Eq. (4.40) becomes

$$
\ln \left(\frac{1}{\bar{x}}\right)=\frac{1+8 \bar{x}^{2}+4 \bar{x}^{3}+8 \bar{x}^{4}+12 \bar{x}^{5}+2 \bar{x}^{6}}{6 \bar{x}^{2}\left(1+2 \bar{x}+4 \bar{x}^{2}+9 \bar{x}^{3}+4 \bar{x}^{4}\right)} .
$$

Solving this equation numerically yields two roots,

$$
\bar{x}_{-} \simeq 0.588, \quad \bar{x}_{+} \simeq 0.439,
$$

which implies that a nonzero $M$ exists in the interval $T_{-}$ $<T<T_{+}$, where

$$
T_{-} / J_{2} \simeq 1.214, \quad T_{+} / J_{2} \simeq 1.885 \text {. }
$$

We may similarly establish the existence on the triangular lattice. As discussed in Sec. II, the six- and seven-spin plaquettes shown in Fig. 6 have inequivalent sites with, in general, unequal magnetizations. This effect should be small, however, and we begin by simply taking $H_{\text {in }}=H_{\text {out }} \equiv H$ and $M_{\text {in }}=M_{\text {out }} \equiv M$ in the plaquette free energies (C6) and (C9). We also begin by setting $K=0, h_{2}=0$, and $\lambda_{2}=1$.

For the six spin plaquette the free energy is given by Eq. (C6) and for these parameters one finds $z_{o}=z_{i}=x^{5}$, and the equation to be solved is

$$
\begin{aligned}
\frac{5}{6} \ln (1 / \bar{x})= & \left(1+12 \bar{x}^{3}+6 \bar{x}^{5}+30 \bar{x}^{6}+6 \bar{x}^{7}+24 \bar{x}^{8}+22 \bar{x}^{9}\right. \\
& \left.+18 \bar{x}^{10}+24 \bar{x}^{11}+12 \bar{x}^{13}+2 \bar{x}^{15}\right) /\left(12 \bar{x}^{3}+24 \bar{x}^{5}\right. \\
& +96 \bar{x}^{6}+216 \bar{x}^{8}+150 \bar{x}^{9}+108 \bar{x}^{10}+384 \bar{x}^{11} \\
& \left.+300 \bar{x}^{13}+72 \bar{x}^{15}\right) .
\end{aligned}
$$

The solutions are

$$
\bar{x}_{-} \simeq 0.796, \quad \bar{x}_{+} \simeq 0.547
$$

corresponding to upper and lower critical temperatures

$$
T_{-} / J_{2} \simeq 1.657, \quad T_{+} / J_{2} \simeq 4.372 .
$$

Similarly, for the seven-spin plaquette the free energy is given by Eq. (C9) and for these parameters one finds $z=x^{4}$, and the equation to be solved becomes 


$$
\begin{aligned}
\frac{4}{7} \ln (1 / \bar{x})= & \left(1+2 \bar{x}^{2}+12 \bar{x}^{3}+24 \bar{x}^{5}+28 \bar{x}^{6}+18 \bar{x}^{7}+58 \bar{x}^{8}\right. \\
& +12 \bar{x}^{9}+9 \bar{x}^{10}+24 \bar{x}^{11}+20 \bar{x}^{12}+24 \bar{x}^{-13} \\
& \left.+8 \bar{x}^{14}\right) /\left(2 \bar{x}^{3}+12 \bar{x}^{3}+96 \bar{x}^{5}+108 \bar{x}^{6}+54 \bar{x}^{7}\right. \\
& +550 \bar{x}^{8}+192 \bar{x}^{9}+900 \bar{x}^{10}+600 \bar{x}^{11}+522 \bar{x}^{12} \\
& \left.+480 \bar{x}^{13}+98 \bar{x}^{14}\right) .
\end{aligned}
$$

The solutions are

$$
\bar{x}_{-} \simeq 0.848 \text { and } \quad \bar{x}_{+} \simeq 0.520 \text {, }
$$

corresponding to upper and lower critical temperatures

$$
\frac{T_{-}}{J_{2}} \simeq 1.528, \quad \frac{T_{+}}{J_{2}} \simeq 6.075
$$

As claimed in the Introduction, the reentrant transition does indeed occur on the triangular lattice, despite the absence of a reconstructed phase. We have chosen values for various fudge factors in a somewhat arbitrary manner, so it is difficult to make quantitative comparisons between the three calculations. It is nevertheless clear that the two calculations on the triangular lattice yield similar results, and that reentrant layering on the triangular lattice occurs over a much larger interval of temperatures than on the square lattice. The reentrant layering is driven by an entropic preference for a disordered top layer, despite the energetic preference for a flat interface. This disordering entropy is presumably larger for the triangular lattice because the second-neighbor interaction divides the lattice into three independent pieces instead of only two, leading to a floppier, more loosely bound interface. The absence of a reconstructed phase therefore actually enhances the reentrant transition.

Translated into the language of layering phenomena, for $T_{-}<T<T_{+}$the layer thickness will vary continuously with chemical potential for top layer coverages in the interval $-1+M_{n-1}<x<-M_{n}$, i.e., for some interval around halffilling of the $n$th layer, where $M_{n}$ is the "magnetization", computed above for the spin-1 model centered on the $n$th layer. However, when the coverage reaches $x=-M_{n}$ it will jump discontinuously, via a first-order phase transition, to a coverage of $x=M_{n}$, i.e., a partially filled $(n+1)$ st layer. More accurately, going beyond the spin-1 approximation, the RSOS model would yield asymmetric magnetizations, $M_{n}^{-}$ and $M_{n}^{+}$, with a jump between $x=-M_{n}^{-}$and $x=M_{n}^{+}$. The discontinuities are, therefore, centered on integer coverages, as seen in the experiments. ${ }^{11-13}$ The reentrant interval will shrink as $h_{2}$ increases. It will shrink as well as $K$ increases to positive values, but grow as $K$ decreases to negative values. On the square lattice, for sufficiently large negative $K$, as we have seen in the previous subsection, the various antiferromagnetic phases will appear (see also the central phase diagram in Fig. 1). On the triangular lattice the DOF phase presumably survives for arbitrarily negative $K$ (see Fig. 2). One can imagine situations where a reconstructed phase with coverage $x_{R}$ different from $\frac{1}{2}$ is stabilized at negative $K$. In this case, presumably, $M$ will continuously approach $x_{R}$, rather than $\frac{1}{2}$, at the phase boundary between the reentrant and reconstructed phases. This clearly does not qualitatively (though it will quantitatively) affect the reentrant phase at positive $K$. In particular, first-order jumps in coverage will always be centered near integer coverage no matter what the nature of the potential reconstructed phase.

The essential message that emerges from these computations is that the interactions that give rise to preroughening of a bulk crystal interface do indeed lead to layering transitions in films between states with roughly half-integer coverage, and that the first-order layering lines are reentrant, with well-defined upper and lower critical points $T_{n}^{2}$ and $T_{c, n}$, as shown in Fig. 1(b).

\section{Beyond spin-1: a correspondence between microscopic and sine-Gordon theories}

We have seen that all of the essential physics of the various phases can be understood qualitatively by considering only three layers. However, in order to see how the various layering transitions evolve as the film grows thicker we must include many more layers. By keeping an effectively infinitely large number of layers, a mean-field phase diagram of the bulk interface may also be worked out and compared to the predictions of the sine-Gordon theory. One way to do this is to generalize the results of Appendix $\mathrm{C}$ and simply enumerate (numerically if necessary) all possible surface configurations for an ever increasing number of layers. However, for thick films one may avoid such a tedious procedure by considering a very special substrate potential that allows one to do everything analytically. In so doing we shall discover a very nice correspondence between the plaquette mean-field theory and the sine-Gordon theory. In some sense the plaquette computation may be viewed as a single renormalization-group transformation that already generates parameters $y_{0}, u_{0}$, and $\kappa_{0}$ for input into Eqs. (3.2) and (3.3). We have done numerical enumerations using the proper van der Waals substrate potential only in order to explore the detailed structure in very thin films.

So far we have discussed two rather different approaches to the study of surface phase transitions. We began our study by looking at solid-on-solid models. These are microscopic system-dependent models, with parameters like the nearestand next-nearest-neighbor interaction strengths, lattice structure, chemical potential, substrate strength, etc. Such an approach is useful when detailed comparisons with experiment or first-principles simulations are to be made.

In Sec. III we adopted a different point of view: since much of the interesting physics should be amenable to a long-wavelength coarse-grained description, we examined a sine-Gordon Hamiltonian (3.1) with partially renormalized parameters $y_{0}, u_{0}, K_{0}$ and an effective substrate potential $V_{0}[h]$. One may find the renormalization-group flow equations for this model and then obtain detailed information about the manner in which the roughening and preroughening critical points of a film approach bulk behavior as the film thickens. Moreover, simple assumptions about the behavior of $y_{0}(T)$ (namely, that it changes sign at some temperature $T_{c}$ ) and about $u_{0}$ lead to phase diagrams that match qualitatively those obtained from the experimental data.

In order to use the sine-Gordon-type Hamiltonian for specific microscopic systems, a method of mapping the discrete 
lattice-based parameters of the RSOS model onto the partially renormalized parameters of the sine-Gordon (SG) Hamiltonian is needed. The renormalization connection between the eight-vertex model (which includes the BCSOS model of roughening - see Appendix D) and the Gaussian model has been studied in Refs. 25 and 26. The main goal of these studies was to find which Gausssian operators are generated by specific eight-vertex operators. While this approach yields much useful information about the relevance of specific operators and about the universality class of the Hamiltonians, it does not provide an explicit mapping between the RSOS Hamiltonian and the corresponding Gaussian Hamiltonian. The restricted SOS condition further complicates attempts to find the precise correspondence between the microscopic and coarse-grained models. As we shall see, however, the mean-field approximation does make it possible to find an approximate mapping between the RSOS and the SG models.

\section{Single-site theory}

We first formulate the single-site mean-field theory of the bulk crystal-vapor interface (no substrate). The Hamiltonian is

$$
\begin{aligned}
\overline{\mathcal{H}} & =\frac{1}{2} K \sum_{\langle i, j\rangle}\left(s_{i}-s_{j}\right)^{2}+\frac{1}{2} L \sum_{(i, k)}\left(s_{i}-s_{k}\right)^{2} \\
& =\alpha \sum_{i} s_{i}^{2}-K \sum_{\langle i, j\rangle} s_{i} s_{j}-L \sum_{(i, k)} s_{i} s_{k},
\end{aligned}
$$

where $\alpha=\left(q_{1} K+q_{2} L\right) / 2$. The formalism of Appendix A leads to the single-site free energy

$$
f_{\mathrm{MF}}(g)=-\alpha[\sigma(g)-g]^{2}-\ln \left[\sum_{s=-\infty}^{\infty} e^{-\alpha(s-g)^{2}}\right]
$$

where $g=H_{i} / 2 \alpha$ (the $H_{i}$, clearly all equal here, were defined in Appendix A), and

$$
\sigma(g)=\left(\sum_{s=-\infty}^{\infty} s e^{-\alpha(s-g)^{2}}\right) /\left(\sum_{s=-\infty}^{\infty} e^{-\alpha(s-g)^{2}}\right)
$$

results from the second saddle-point equation (A9). Physically, $\sigma(g)$ corresponds to the equilibrium film thickness. In the absence of a substrate the free energy will have an infinite sequence of minima reflecting the perfect periodicity of the system: $f_{\mathrm{MF}}(g+n)=f_{\mathrm{MF}}(g)$ for any integer $n$. Thus if $g_{0}$ is a minimum, so is $g_{0}+n$. In order to retain this periodicity we avoid truncating the sum over $s$ in Eq. (4.51) by using the Poisson summation formula,

$$
\sum_{k=-\infty}^{\infty} f(k)=\sum_{m=-\infty}^{\infty} \int_{-\infty}^{\infty} d x f(x) e^{-i 2 \pi m x}
$$

which then yields

$$
\sum_{s=-\infty}^{\infty} e^{-\alpha(s-g)^{2}}=\sqrt{\pi / \alpha}\left[1+\sum_{n=1}^{\infty} 2 e^{-n^{2} \pi^{2} / \alpha} \cos (2 \pi n g)\right] .
$$

The factor $e^{-n^{2} \pi^{2} / \alpha}$ in the sum guarantees that this new series is very rapidly convergent and for most purposes it is adequate to keep the first few terms, each of which is manifestly periodic in $g$. Thus we have

$$
\begin{aligned}
f_{\mathrm{MF}}(g)= & -\frac{1}{2} \ln (\pi / \alpha)-y_{R} \cos (2 \pi g)-u_{R} \cos (4 \pi g) \\
& +O\left(y_{R}^{9}\right),
\end{aligned}
$$

where $y_{R}=2 e^{-\pi^{2} / \alpha}$ and $u_{R}=O\left(y_{R}^{4}\right) \ll y_{R}$. It is clear that in the single-site mean-field theory $y_{R}$ is always positive so that $f_{\mathrm{MF}}$ is minimized for integer valued $g$. Thus the film is always globally flat with integer valued thickness, and there is no possibility of either a rough phase or a disordered flat phase. We shall see below that the plaquette mean-field theory does give a DOF phase, but even there an exponentially small corrugation remains to arbitrarily high temperature (i.e., small $\alpha$ ). This is an artifact of the mean-field approximation, which misses entirely the subtle features of the roughening transition. However, for the purposes of estimating phase boundaries, as we have done in Figs. 1 and 2, one may define the roughening temperature as the point where the corrugation falls below some small value, $y_{R}^{\mathrm{min}}$. In Fig. 1 the vertical roughening line running through the points $L$ and $M$ has actually been taken for convenience from the exact solution of the six vertex model (discussed in Appendix D), while that running from the $K=0$ axis to the point $L$ has been estimated from the mean-field theory using $y_{R}^{\min }$ $=10^{-6}$ (essentially the limit of our numerical resolution). The roughening line in Fig. 2 uses the same $y_{R}^{\min }$, but, in order to give a better feel for the errors involved, the width of the line corresponds to $20 \%$ adjustments to either side of this. Clearly the ambiguities are greatest close to the preroughening line.

\section{Plaquette theory}

We shall next show that Eq. (4.55) remains valid for the square lattice using a four-site mean-field theory, but that $y_{R}$ may now change sign precisely as indicated in Fig. 12, thus exhibiting a DOF phase. We confine the discussion to $J_{1}$ $>0$, though we have also carried out a more involved calculation, which we shall not detail here, for $J_{1}<0$, to investigate reconstructed phases. We will also now include a substrate potential in order to exhibit layering.

The Hamiltonian is exactly as in Eq. (4.50) except that we now add a term $\frac{1}{2} \kappa \Sigma_{i}\left(s_{i}-h_{0}\right)^{2}$, where $\kappa$ models the strength of the effective substrate potential and $h_{0}$ determines the film thickness. We shall see that this quadratic form of the substrate potential still permits an analytic analysis. Following the formalism in Appendix A, we tile the lattice with $2 \times 2$ plaquettes and rewrite the Hamiltonian in the form $\overline{\mathcal{H}}$ $=\Sigma_{P}\left[\overline{\mathcal{H}}_{P}+\overline{\mathcal{H}}_{\text {interplaq }}\right]$ where $P$ denotes a sum over plaquettes and (in the notation of Fig. 7), 


$$
\begin{aligned}
\overline{\mathcal{H}}_{P}= & \frac{1}{2} K\left[\left(s_{P 1}-s_{P 2}\right)^{2}+\left(s_{P 2}-s_{P 3}\right)^{2}+\left(s_{P 3}-s_{P 4}\right)^{2}\right. \\
& \left.+\left(s_{P 4}-s_{P 1}\right)^{2}\right]+\frac{1}{2} L\left[\left(s_{P 1}-s_{P 3}\right)^{2}+\left(s_{P 2}-s_{P 4}\right)^{2}\right] \\
& +\left(K+\frac{3}{2} L\right)\left(s_{P 1}^{2}+s_{P 2}^{2}+s_{P 3}^{2}+s_{P 4}^{2}\right)+\frac{1}{2} \kappa\left[\left(s_{P 1}-h_{0}\right)^{2}\right. \\
& \left.+\left(s_{P 2}-h_{0}\right)^{2}+\left(s_{P 3}-h_{0}\right)^{2}+\left(s_{P 4}-h_{0}\right)^{2}\right],
\end{aligned}
$$

and $\overline{\mathcal{H}}_{\text {interplaq }}$ contains terms such as $-(K / 2) s_{P 1} s_{P_{3} 2}$ and

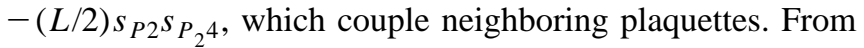
Appendix A, the mean-field free energy per site is then found to be

$$
f_{\mathrm{MF}}=-\left(K+\frac{3}{2} L\right) \sigma^{2}+g \sigma-\frac{1}{4} \log \left(Z_{\mathrm{Bog}}\right)
$$

where

$$
Z_{\mathrm{Bog}}=\sum_{s_{1}, s_{2}, s_{3}, s_{4}}^{\prime} \exp \left[-\overline{\mathcal{H}}_{P}+g\left(s_{1}+s_{2}+s_{3}+s_{4}\right)\right]
$$

is an effective plaquette partition function and $\sigma(g)$ $=\frac{1}{4} \partial \ln Z_{\mathrm{Bog}} / \partial g$ is again the average film thickness. The prime on the summation is a reminder that the RSOS condition must be obeyed. If $s_{1}$ is considered as an unconstrained variable, then for $s_{1}=n$, this condition allows only 19 different configurations for the other variables. The energy $\epsilon_{i}$ of configuration $i$ may be written in the form $\epsilon_{i}=a_{i} n^{2}+b_{i} n$ $+c_{i}$. For example, if all four spins take value $n$ we have $a_{1}=4 K+6 L+2 \kappa, b_{1}=-4 g-4 h_{0} \kappa$ and $c_{1}=2 \kappa h_{0}^{2}$. The partition function therefore takes the discrete Gaussian form,

$$
Z_{\mathrm{Bog}}=\sum_{i=1}^{19} \sum_{n=-\infty}^{\infty} e^{-a_{i} n^{2}-b_{i} n-c_{i}}
$$

Each sum over $n$ can be reexpressed in terms of periodic functions using Eq. (4.54) with the result

$$
\begin{aligned}
Z_{\mathrm{Bog}}= & \sqrt{2 \pi / \Lambda} e^{-2 \kappa h_{0}^{2}+\mu^{2} / 2 \Lambda}[A+B \cos (2 \pi \mu / \Lambda) \\
& +C \cos (4 \pi \mu / \Lambda)+\cdots],
\end{aligned}
$$

where

$$
\begin{gathered}
\Lambda=8 K+12 L+4 \kappa \\
\mu=4 g+4 h_{0} \kappa \\
A=1+4 \exp [-(\kappa+4 K+5 L)]+4 \exp [-(\kappa+4 K+5 L) / 2] \\
+2 \exp [-(\kappa+6 K+3 L) / 2] \\
+8 \exp [-(3 \kappa+14 K+13 L) / 8] \\
B=2 \exp \left(-2 \pi^{2} / \Lambda\right)(1+4 \exp [-(\kappa+4 K+5 L)] \\
-4 \exp [-(\kappa+4 K+5 L) / 2] \\
-2 \exp [-(\kappa+6 K+3 L) / 2])
\end{gathered}
$$

$$
\begin{aligned}
C= & 2 \exp \left(-8 \pi^{2} / \Lambda\right)(1+4 \exp [-(\kappa+4 K+5 L)] \\
& +4 \exp [-(\kappa+4 K+5 L) / 2]+\exp [-(\kappa+6 K+3 L) / 2] \\
& -8 \exp [-(3 \kappa+14 K+13 L) / 8]) .
\end{aligned}
$$

From Eqs. (4.57) and (4.59) we find

$$
\begin{aligned}
f_{\mathrm{MF}}(\theta)= & f_{0}-y_{R} \cos (2 \pi \theta)-u_{R} \cos (4 \pi \theta)+\frac{1}{2} \kappa\left[\theta-h_{0}\right. \\
& -d(\theta)]^{2},
\end{aligned}
$$

where $f_{0}$ is a constant. This free energy is now in the sineGordon form. A more convenient variational parameter $\theta$ $=4 g+h_{0} \kappa / \Lambda$ is introduced here, and we have defined

$$
d(\theta)=\left(2 \pi y_{R} / \Lambda\right) \sin (2 \pi \theta)+\left(4 \pi u_{R} / \Lambda\right) \sin (4 \pi \theta) .
$$

The sine-Gordon parameters may now be expressed in terms of $K, L$, and $\kappa$ as

$$
\begin{gathered}
y_{R}=\frac{B}{4 A}+O\left[\left(\frac{B}{A}\right)^{3}\right] \\
u_{R}=\frac{C}{4 A}-\left(\frac{1}{16}+\frac{\pi^{2}}{8 \Lambda}\right)\left(\frac{B}{A}\right)^{2}-\frac{1}{64}\left(\frac{B}{A}\right)^{4}+O\left[\left(\frac{B}{A}\right)^{5}\right] .
\end{gathered}
$$

Once $\theta\left(h_{0}, g\right)$ is determined by minimizing $f_{\mathrm{MF}}$, the film thickness is given by $\sigma=\theta-d(\theta)$.

It is not hard to see that $y_{R}$ is positive at low temperature (large $K=J_{1} / T$ and $L=J_{2} / T$ ), but at high temperatures it changes sign: although Eq. (4.61) implies that $A$ is always positive, $B$, and hence $y_{R} \approx B / 4 A$, change sign. This confirms the existence of the DOF phase. Those portions of Fig. 1 that do not involve reconstruction are based mainly on numerically mapping out the surface and layering phase diagrams using Eq. (4.57) [with the exception of Fig. 1(d), whose $\theta$ DOF phase, as we have emphasized, does not appear in this model; we have substituted the phase diagram computed from the sine-Gordon model in this case]. However, for smaller film thicknesses we have also used the more realistic van der Waals substrate potential shown in Fig. 3 and numerically enumerated the surface configurations to compute the mean-field free energy. The phase diagrams involving reconstruction obviously require more than a single $H_{i}$ and we have applied the appropriate generalizations of Eq. (4.57) to their computation, again using numerical enumeration and a more realistic substrate potential at smaller film thicknesses.

If we view $\theta$ as a new renormalized continuous spin, replacing the four original spins, then $f_{\mathrm{MF}}$ represents a corrugation plus substrate potential that we should substitute into Eq. (3.2). Notice that the effective substrate part has been modified slightly by the periodic terms. Missing from this analysis is an estimate for the interplaquette coupling $K_{0}$. Thus a true renormalization-group transformation would also generate renormalized couplings between the plaquettes. We have not looked into this, but simple estimates could presum- 
ably be made by comparing the estimated roughening temperatures derived from the plaquette calculation and the single-site theory, Eq. (4.55).

\section{CONCLUSIONS}

In this last section we briefly compare the theoretical results we have obtained from the RSOS and sine-Gordon models with the results of the experiments on noble gases on graphite substrates ${ }^{11-14,18}$ and discuss other possible interpretations of the data. We end by discussing work for the future.

\section{A. Comparison with experiment}

If we accept the premise that the RSOS model indeed captures the essential physics of the thin-film equilibria, and that the experimental measurements have not missed any significant features in the phase diagram, then it is difficult not to conclude that the reentrant layering is indeed a reflection of the DOF phase on the bulk interface. Thus, although Figs. $1(\mathrm{~g}, \mathrm{~h})$, which involve reconstruction, show phase diagrams remarkably similar to Fig. 1(c) there are also distinct differences. In Fig. $1(\mathrm{~g})$ the experimental vapor pressure isotherms will have steps at the wrong coverages, which seems to be ruled out by the experimental data. Similarly in Fig. 1(h), although the steps in the vapor pressure isotherms now occur at the correct coverages, there is a film analogue of the transition from the rough to reconstructed-rough phase at higher temperatures that is not seen in the experiments. This transition is second order, rather than first order, so it might be more difficult to see. Both these scenarios, however, leave open the question of what kind of triangular lattice reconstructed and reconstructed-rough phases might replace the square lattice checkerboard phase. Direct probes of the surface structure through scattering measurements would be required to see if, in fact, the upper layer of the film has nontrivial spatial order.

On the other hand, accepting the premise that the DOF phase is responsible for the reentrant layering, and the fact that there is not expected to be a reconstructed phase on the triangular lattice, we have seen that the phase boundary between the flat and DOF phases extends, in principle, to arbitrarily large $J_{2} / J_{1}$. However, the Kosterlitz-Thouless theory tells us that only a finite segment of this boundary can be a continuous transition. The majority of this boundary must therefore be first order, and in retrospect it may not be too surprising then that the experimental data show evidence of a first-order preroughening transition.

One might be concerned by the fact that the real underlying lattice structure of argon on graphite is fcc rather than triangular. As mentioned in the Introduction, this means that although individual layers indeed form two-dimensional triangular lattices, they do not lie directly on top of one another, but are displaced horizontally from one another so that subsequent layers lie in the interstices of the preceding ones. In principle, this will affect the quantitative predictions of the RSOS model. This certainly should be checked, ${ }^{19}$ but all evidence so far indicates that the results are not particularly sensitive to lattice structure. In the present work we have considered both square and triangular lattices while, for example, the original work of Rommelse and den Nijs was based on a bcc lattice. ${ }^{2,3}$
Another possibility is that a lattice model is simply insufficient for describing the properties of the film. Such would be the case, for example, if two-dimensional melting were to occur. The lattice model cannot describe a phase where incommensurability effects occur, i.e., when the film is in a floating solid phase, with a lattice structure incommensurate with that of the substrate. Such phases indeed occur in very thin films: the data in Fig. 1 of Ref. 17 clearly show twodimensional melting lines, as well as triple points where twodimensional liquid, vapor, and solid coexist, in the first two or three layers of argon on graphite. The RSOS model is clearly inadequate if such phases were to persist in the upper layers of arbitrarily thick films.

In a recent letter, Phillips, Zhang, and Larese ${ }^{18}$ (PZL) take precisely this point of view. They report a Monte Carlo simulation of up to several thousand Lennard-Jones argon atoms on a two-dimensional substrate, with an extent such that about 1000 atoms fill one layer, and studied films up to about three layers thick. They found the usual layering transitions at lower temperatures, and smooth, continuous growth of the film at higher temperatures. However, at intermediate temperatures they found, as a function of increasing coverage (or, equivalently, increasing chemical potential) at fixed temperature, a sudden increase in the occupation of the fourth layer at the expense of the occupation of the third layer just before third layer completion. This is accompanied by a positional disordering of the third layer, which is interpreted as a melting transition. As more particles are added, the density in the third layer increases again, and at a nominal coverage of about 3.5 layers the third layer apparently resolidifies. This resolidification, apparently induced by the hydrostatic pressure of the particles above due to the binding energy of the substrate, is argued to give rise to the steps in the vapor pressure isotherms in the reentrant layering regime. This process is argued to repeat itself layer by layer as the film grows. Since their scenario involves both liquid and solid phases in the film PZL question the use ${ }^{17}$ of the RSOS lattice model.

There are various problems with this scenario. ${ }^{27}$ First of all, the behavior of the third layer is rather different from that of higher layers, where our DOF phase interpretation is claimed to be valid, and is, therefore, not a good basis for generalization. Thus, although the first and second layers of argon have independent two-dimensional solid, liquid, and gas phases, complete with critical points, triple points, and melting transitions, the fourth, fifth, and sixth layers behave rather differently. In particular, they do not have triple points or two-dimensional liquid-gas critical points, but they do have low-temperature layering transitions at integer layer coverages, and higher-temperature "reentrant" layering transitions at half-integer coverages, zipped to the lowtemperature layering transitions by the zigzagging line of heat capacity peaks. The third layer, on the other hand, is an intermediate case, showing both types of behavior: there is a two-dimensional triple point and a two-dimensional critical point, but there is also the first reentrant layering transition, marked by coexistence between 2.5 and 3.5 layers, which entrains the melting of the third layer. It is not surprising, then, that PZL see evidence of melting associated with that rather complicated situation, but the very different nature of 
the phase diagram for thicker films makes us skeptical of the generalizations they draw from that observation.

There are also two quantitative reasons for doubting the PZL scenario in third and higher layers. First, if they were due to solidification we would expect the vertical steps in adsorption isotherms that are the signature of the phenomenon to be roughly $10 \%$ of a layer in height, the typical density difference between liquids and solids (note, in fact, that for continuous two-dimensional melting there is no density difference at all). Instead, all of the data, including PZL's own isotherms, consistently show steps of roughly a full layer. Second, the hydrostatic pressure that is supposed to induce the transition is negligible in the third layer and smaller yet in higher layers. This point shows up clearly in the energetics: the binding energy of the third layer is little more than $k_{B} T / 10$, and decreases as the inverse cube of the film thickness. The canonical ensemble simulation method used by these authors does not allow a direct reconstruction of the isotherms, so no prediction is given for the size of the discontinuous step, nor is any other direct thermodynamic evidence given for this freezing transition. The apparent absence of melting phenomena leads us to believe that the RSOS model provides an adequate description of the thicker films in which the physics approaches that of the bulk interface. The DOF phase predicted by this model then produces the full step reentrant layering transitions (coexistence between $n+\frac{1}{2}$ and $n-\frac{1}{2}$ layers). This, along with the natural explanation of the zipper in terms of a first-order preroughening transition, demonstrates that the RSOS model has remarkable descriptive powers and the agreement of its predictions with the experimental data is striking. Its very simplicity, which is a shortcoming in thinner films, becomes a virtue in thicker films.

\section{B. Future work}

Given the RSOS model parameters $J_{1}$ and $J_{2}$ the theory developed in the present work then allows reasonable estimates of the renormalized sine-Gordon parameters $y$ and $u$ that determine the actual phase boundaries. Perhaps the largest gap in our theoretical understanding of the reentrant layering phenomenon is the connection between the microscopic interparticle interactions and these effective RSOS model parameters. If one models the particles, as in Ref. 18, using a Lennard-Jones potential with hard core radius $\sigma$ and attractive minimum depth $-\varepsilon$, the question is whether there is a reasonably well-defined mapping $J_{1}=J_{1}(\sigma, \varepsilon, T)$ and $J_{2}=J_{2}(\sigma, \varepsilon, T)$, and if so what range of $J_{1}$ and $J_{2}$ the mapping covers for physically motivated ranges of $\sigma$ and $\varepsilon$. In particular, can the effective $J_{2}$ be made small enough to produce continuous preroughening, and do any of the corresponding Lennard-Jones potentials match that of a real material? Answering this question theoretically would require extending the PZL simulations to other Lennard-Jones potentials besides that of argon and to much thicker films.

Another point is that we have seen that the onecomponent RSOS model we study does not produce a $\theta$ DOF phase. The two-component BCSOS model does have a $\theta$ DOF phase but there would be considerable experimental difficulty in investigating such a two-adsorbate system assuming that one existed. If this phase is, in fact, experimen- tally realizable for a system with a single-adsorbate species (as opposed to "alloys" - see Appendix D) then we conclude, at the very least, that something beyond an RSOS model with only first- and second-neighbor interactions is required. One can therefore ask: What potential would be required in order to generate a $\theta \mathrm{DOF}$ phase in a onecomponent system?

To conclude, recent experiments have shown that there is much new interesting physics to be found in thin-film and bulk interface studies. The present work will hopefully motivate future experimental efforts in search of the as yet unseen phases and phase diagrams that we have found.

\section{ACKNOWLEDGMENTS}

We thank Peter Day, David Goodstein, and David Huse for enlightening conversations. The support of the NSF through Grant No. DMR-9308205 is gratefully acknowledged.

\section{APPENDIX A: PLAQUETTE MEAN-FIELD THEORIES}

In this appendix we outline the general formalism for constructing consistent mean-field theories, using plaquettes of arbitrary size, based on any given Hamiltonian. By consistent we mean that the mean-field free energy should obey all thermodynamic principles. We guarantee this by demonstrating that the mean-field theory becomes exact for a limiting case of a certain model Hamiltonian closely related to the original given one. The formalism we present here is a fairly straightforward generalization of that described in Ref. 28.

The idea is to treat each plaquette as a single site with a set of internal variables, each of which may interact with the internal variables on other plaquettes. If we label the plaquettes by an index $P$, we denote the complete set of internal variables by $\left\{S_{P \alpha}\right\}, \alpha=1, \ldots, K$. Often the different plaquettes will be identical copies of one another, but this is not assumed in general. The internal variables will include, for example, not only the height variables $h_{i}$ within the plaquette $P$, but also all powers and products of them, $h_{i}^{2}$, $h_{i}^{3}, h_{i} h_{j}, h_{i}^{2} h_{j} h_{k}^{3}$ (with $i, j$, and $k$ all in $P$ ), etc. We consider then a rather general reduced Hamiltonian, $\overline{\mathcal{H}}=\mathcal{H} / k_{B} T$, of the form

$$
\overline{\mathcal{H}}=\sum_{P} \overline{\mathcal{H}}_{P}\left\{S_{P \alpha}\right\}-\sum_{P, \alpha} h_{P \alpha} S_{P \alpha}+\mathcal{A}\left\{S_{P \alpha}\right\},
$$

where $\overline{\mathcal{H}}_{P}\left\{S_{P \alpha}\right\}$ depends only on the internal variables in plaquette $P$, and the conjugate fields $h_{P \alpha}$ should not be confused with the original height variables $h_{i}$. If the plaquettes are identical $\overline{\mathcal{H}}_{P}$ will not depend on $P$. The potential $\mathcal{A}$ contains all interactions between different plaquettes. These interactions are forbidden from containing products of the $S_{P \alpha}$ within the same plaquette $P$. Technically this means that the derivative $\partial \mathcal{A} / \partial S_{P \alpha}$ is independent of $S_{P \beta}$ for all $\beta$ $=1, \ldots, K$, and hence that $\mathcal{A}$ is a sum of terms multilinear in the $S_{P \alpha}$. From a practical point of view this means that a term like $\left(h_{i}-h_{j}\right)^{2}$ must be multiplied out so that $h_{i}^{2}$ and $h_{j}^{2}$ are included in $\overline{\mathcal{H}}_{P}$ for their respective plaquettes, while the cross term $h_{i} h_{j}$ is included in $\mathcal{A}$ (assuming that $i$ and $j$ lie in 
different plaquettes, otherwise the entire term belongs in $\overline{\mathcal{H}}_{P}$ ). The conjugate fields $h_{P \alpha}$ are introduced in a term separate from $\overline{\mathcal{H}}_{P}$ and $\mathcal{A}$ for later convenience. The partition function,

$$
Z\left\{h_{P \alpha}\right\}=\int D S e^{-\overline{\mathcal{H}}\left\{S_{P \alpha}\right\}},
$$

is then a functional integral over some fundamental field $S$ out of which the $S_{P \alpha}$ are constructed. The reduced free energy is $F / k_{B} T \equiv \bar{F}=-\ln (Z)$.

We now introduce independent continuous variables $\sigma_{P \alpha}$ and their conjugate fields $H_{P \alpha}$ as follows: we first use the variables $\sigma_{P \alpha}$ to represent the variables $S_{P \alpha}$ simply by introducing appropriate $\delta$ functions:

$$
\begin{aligned}
Z\left\{h_{P \alpha}\right\}= & \int D S e^{-\Sigma_{P} \overline{\mathcal{H}}_{P}\left\{S_{P \alpha}\right\}} \int D \sigma \prod_{P, \alpha} \delta\left(\sigma_{P \alpha}-S_{P \alpha}\right) \\
& \times e^{-\mathcal{A}\left\{\sigma_{P \alpha}\right\}+\Sigma_{P, \alpha} h_{P \alpha} \sigma_{P \alpha}} .
\end{aligned}
$$

We then introduce the $H_{P \alpha}$ by using the usual Fourier representation of the $\delta$ function:

$$
\delta(\sigma-S)=\int_{C} \frac{d H}{2 \pi i} e^{H(\sigma-S)}
$$

where the integral is over a vertical contour $C$, extending from $c-i \infty$ to $c+i \infty$ in the complex $H$ plane, where $c$ is an arbitrary real number, which will later be chosen for convenience to satisfy a certain saddle point condition. If we define the single plaquette reduced free energies $\Phi_{P}$ via

$$
e^{-\Phi_{P}\left\{H_{\alpha}\right\}} \equiv \int D S e^{-\overline{\mathcal{H}}_{P}\left\{S_{\alpha}\right\}-\Sigma_{\alpha} H_{\alpha} S_{\alpha}},
$$

then the partition function may be written

$$
Z_{n}\left\{h_{P \alpha}\right\}=\int D H \int D \sigma e^{-n \mathcal{F}\left\{H_{P \alpha}, \sigma_{P \alpha} ; h_{P \alpha}\right\}},
$$

where $n=1$, but for convenience has been introduced as a free parameter, and the free-energy functional is

$$
\begin{aligned}
\mathcal{F}\left\{H_{P \alpha}, \sigma_{P \alpha} ; h_{P \alpha}\right\} \equiv & \sum_{P} \Phi_{P}\left\{H_{P \alpha}\right\}-\sum_{P, \alpha}\left(H_{P \alpha}+h_{P \alpha}\right) \sigma_{P \alpha} \\
& +\mathcal{A}\left\{\sigma_{P \alpha}\right\} .
\end{aligned}
$$

We now consider the saddle-point approximation, which becomes exact in the limit $n \rightarrow \infty$ : define the mean-field reduced free energy,

$$
\bar{F}_{\mathrm{MF}}\left\{h_{P \alpha}\right\}=\mathcal{F}\left\{H_{P \alpha}^{0}, \sigma_{P \alpha}^{0} ; h_{P \alpha}\right\},
$$

where $\left\{H_{P \alpha}^{0}, \sigma_{P \alpha}^{0}\right\}$ satisfy the saddle-point equations

$$
\begin{gathered}
\left(\frac{\partial \mathcal{F}}{\partial H_{P \alpha}}\right)_{0}=0 \Rightarrow \sigma_{P \alpha}^{0}=\left(\frac{\partial \Phi_{P}}{\partial H_{P \alpha}}\right)_{0}, \\
\left(\frac{\partial \mathcal{F}}{\partial \sigma_{P \alpha}}\right)_{0}=0 \Rightarrow H_{P \alpha}^{0}+h_{P \alpha}=\left(\frac{\partial \mathcal{A}}{\partial \sigma_{P \alpha}}\right)_{0},
\end{gathered}
$$

where the subscript 0 indicates evaluation at the saddle point. Clearly the solutions must be real, and we may specify the number $c$ in Eq. (A4) to be $H_{P \alpha}^{0}$ for the corresponding contour. We emphasize that because the integration is over complex values of the $H_{P \alpha}, \quad F_{\mathrm{MF}}$ is not in general the minimum of $\mathcal{F}$ over all $H_{P \alpha}$ and $\sigma_{P \alpha}$, not even over all real values of $H_{P \alpha}$ and $\sigma_{P \alpha}$. The direction of steepest descent through the saddle point is often a nontrivial angle in the complex plane. However, if there are multiple saddle points one must obviously choose the one with minimal free energy. We will discuss at the end how to define $F_{\mathrm{MF}}$ through a proper extremum principle. The first equation gives the mean-field approximation for $-\partial F / \partial h_{P \alpha}=\left\langle S_{P \alpha}\right\rangle$ in terms of the effective single plaquette free energy $\Phi_{P}$ while the second equation gives the effective fields $H_{P \alpha}$ acting on plaquette $P$ due to the external field $h_{P \alpha}$ as well as the mean fields $\sigma_{P^{\prime} \alpha^{\prime}}$ on plaquettes $P^{\prime}$ with which it interacts. The latter then serve as inputs to $\Phi$ in the first equation. Notice that

$$
\begin{aligned}
-\frac{\partial \bar{F}_{\mathrm{MF}}}{\partial h_{P \alpha}}= & -\frac{\partial \mathcal{F}}{\partial h_{P \alpha}}-\sum_{P^{\prime} \alpha^{\prime}}\left[\left(\frac{\partial \mathcal{F}}{\partial H_{P^{\prime} \alpha^{\prime}}}\right)_{0} \frac{\partial H_{P^{\prime} \alpha^{\prime}}^{0}}{\partial h_{P \alpha}}\right. \\
& \left.+\left(\frac{\partial \mathcal{F}}{\partial \sigma_{P^{\prime} \alpha^{\prime}}}\right)_{0} \frac{\partial \sigma_{P^{\prime} \alpha^{\prime}}}{\partial h_{P \alpha}}\right]=\sigma_{P \alpha}^{0},
\end{aligned}
$$

where the last equality follows because the saddle-point equations cause the second term to vanish identically. This proves consistency, namely, that $\sigma_{P \alpha}=\left\langle S_{P \alpha}\right\rangle_{\mathrm{MF}}$ is indeed the mean-field average of $S_{P \alpha}$. Consistency is in fact guaranteed by the deeper result that the limit $n \rightarrow \infty$ may be realized as an explicit model: ${ }^{28}$ it is straightforward to show that for general integer $n \geqslant 1$ the partition function $Z_{n}$ may be obtained from the Hamiltonian,

$$
\begin{gathered}
\overline{\mathcal{H}}_{n}=\sum_{l=1}^{n} \sum_{P} \overline{\mathcal{H}}_{P}\left\{S_{P \alpha}^{(l)}\right\}-\sum_{P, \alpha} h_{P \alpha} \Sigma_{P \alpha}+n \mathcal{A}\left\{\frac{1}{n} \Sigma_{P \alpha}\right\}, \\
\Sigma_{P \alpha} \equiv \sum_{l=1}^{n} S_{P \alpha}^{(l)},
\end{gathered}
$$

where $\left\{S_{P \alpha}^{(l)}\right\}_{l=1}^{n}$ are $n$ identical copies of the original $\left\{S_{P \alpha}\right\}$ with identical single plaquette Hamiltonians $\overline{\mathcal{H}}_{0}$ interacting only through their mean values, $\left\{1 / n \Sigma_{P \alpha}\right\}$, which appear in $\mathcal{A}$. The form (A6) follows by introducing the Fourier representation of the $\delta$ functions $\delta\left(n \sigma_{P \alpha}-\Sigma_{P \alpha}\right)$ and integrating out the $\left\{S_{P \alpha}^{(l)}\right\}$ as before. In the limit $n \rightarrow \infty$ the saddle-point equations represent an exact solution to this model.

It is worth reemphasizing that the free energy, Eq. (A8), depends only on the fields, $\left\{h_{P \alpha}\right\}$. Given only $\bar{F}_{\mathrm{MF}}\left\{h_{P \alpha}\right\}$ the 
mean-field averages $\left\{\sigma_{P \alpha}^{0}\right\}$ must be obtained through Eq. (A10). It is sometimes preferable to perform a Legendre transformation and work with a free energy that depends explicitly only on the $\left\{\sigma_{P \alpha}^{0}\right\}$. We define then the Helmholtz free energy,

$$
A_{\mathrm{MF}}\left\{\sigma_{P \alpha}^{0}\right\} \equiv \bar{F}_{\mathrm{MF}}+\sum_{P, \alpha} h_{P \alpha} \sigma_{P \alpha}^{0},
$$

in which Eq. (A10) is used to eliminate the $\left\{h_{P \alpha}\right\}$. Equivalently, we have

$$
A_{\mathrm{MF}}\left\{\sigma_{P \alpha}^{0}\right\}=\sum_{P} \Phi_{P}\left\{H_{P \alpha}\right\}-\sum_{P, \alpha} H_{P \alpha} \sigma_{P \alpha}^{0}+\mathcal{A}\left\{\sigma_{P \alpha}^{0}\right\},
$$

in which the first line of Eq. (A9) is used to eliminate the $\left\{H_{P \alpha}\right\}$ in favor of the $\left\{\sigma_{P \alpha}^{0}\right\}$. The result is explicitly independent of the $\left\{h_{P \alpha}\right\}$, which are then computed from $A_{\mathrm{MF}}$ via

$$
h_{P \alpha}=\frac{\partial A_{\mathrm{MF}}}{\partial \sigma_{P \alpha}^{0}} .
$$

From Eq. (A13) we see that the computation of $A_{\mathrm{MF}}$ from the functional $\mathcal{F}$ given in Eq. (A7) is easier than the computation of $\bar{F}_{\mathrm{MF}}$ since it involves solving only one of the saddle-point equations, Eq. (A9).

One might be concerned about an obvious ambiguity in the definition of $\overline{\mathcal{H}}_{P}$. Clearly terms like $\Sigma_{P, \alpha} h_{P \alpha} S_{P \alpha}$, which are linear in the $S_{P \alpha}$, could also be included in the single plaquette part of the Hamiltonian, thereby changing the form of the single plaquette free energy $\Phi_{P}$. Fortunately the saddle-point equations are insensitive to this ambiguity, ${ }^{28}$ which is easily seen only to result in a corresponding shift in the $\left\{H_{P \alpha}\right\}$ : the sum, $H_{P \alpha}^{0}+h_{P \alpha}$, is unchanged and from Eq. (A9) one immediately sees that the physical quantities $\left\{\sigma_{P \alpha}\right\}$ are therefore unaffected. Notice from the second line of Eq. (A9) that if $\mathcal{A}$ is independent of a particular $S_{P \alpha}$, then one immediately has the solution $H_{P \alpha}=-h_{P \alpha}$. Therefore, unless $S_{P \alpha}$ appears inside a nontrivial interplaquette interaction, one may simply include the term $h_{P \alpha} S_{P \alpha}$ in $\overline{\mathcal{H}}_{P}$ and set the corresponding $H_{P \alpha}$ to zero. Therefore, the number of free minimization parameters $\left\{H_{P \alpha}\right\}$ that need to be introduced depends only on the complexity of $\mathcal{A}$ and not on that of $\overline{\mathcal{H}}_{P}$. For example, if interactions in the roughening model take the form $\left(h_{i}-h_{j}\right)^{2}$, only fields conjugate to the individual $\left\{h_{i}\right\}$ need be introduced since $h_{i}^{2}$ (as well as $h_{i} h_{j}$ for $i$ and $j$ in the same plaquette) appear only in single plaquette terms.

It is worth commenting on the relation between this formalism and the intuitive idea of mean-field theory where one makes a distinction between a particular plaquette of variables, $S_{\alpha}$, which is treated exactly, and its "environment," which then interacts with the $S_{\alpha}$ only through its average properties. In the present formalism these notions are made precise through the distinction between the plaquette free energy, $\Phi$, which contains an explicit trace over the fluctuating internal $S_{P \alpha}$, and the interplaquette interactions $\mathcal{A}$, which contain only the nonfluctuating $\sigma_{P \alpha}$. Now, in the in- tuitive picture it is not obvious precisely what aspects of the average environmental behavior are relevant. For example, suppose the fundamental field has spin $j$, taking values $s_{i}=$ $-j,-j+1, \ldots, j$ on each site $i$ with corresponding equilibrium probabilities $p_{i}\left(s_{i}\right)$. In principle, all of these $2 j$ independent probabilities on each site ought to be determined self-consistently in the mean-field theory. Equivalently, we may determine the mean powers, $\left\langle s_{i}^{m}\right\rangle=\sum_{l=-j}^{j} l^{m} p_{i}(l), m$ $=1, \ldots, 2 j \quad(m=1$ corresponding to the order parameter). Within the formalism, however, the powers $s_{i}^{m}$ must be contained in the $\left\{S_{P \alpha}\right\}$, and their averages contained in the $\left\{\sigma_{P \alpha}\right\}$. The consistency of the theory indeed demands that all of these variables (and more if the plaquettes contain more than one site) enter appropriately, though, as we have seen, great simplifications occur for those that do not appear explicitly in the interplaquette interaction term $\mathcal{A}$.

Finally, in order to define the theory through a true extremum principle, we make the connection to the Bogoliubov method for constructing mean-field theories. The Bogoliubov inequality states that for any two Hamiltonians $\overline{\mathcal{H}}$ and $\overline{\mathcal{H}}_{1}$, with corresponding reduced free energies $\bar{F}$ and $\bar{F}_{1}$,

$$
\bar{F} \leqslant \bar{F}_{1}+\left\langle\overline{\mathcal{H}}-\overline{\mathcal{H}}_{1}\right\rangle_{1},
$$

where the average is with respect to $\overline{\mathcal{H}}_{1}$. The strategy is to pick an appropriate family of exactly soluble model Hamiltonians $\overline{\mathcal{H}}_{1}(\lambda)$ depending on a set of free parameters generically denoted by $\lambda$. One then defines the Bogoliubov meanfield free energy via

$$
F_{\text {Bog }}=\min _{\lambda}\left\{\bar{F}_{1}(\lambda)+\left\langle\overline{\mathcal{H}}-\overline{\mathcal{H}}_{1}(\lambda)\right\rangle_{1}\right\}
$$

Can one connect this procedure to the saddle-point method above? The answer is yes: $F_{\mathrm{Bog}}$ is precisely equal to $\bar{F}_{\mathrm{MF}}$ with the choice

$$
\overline{\mathcal{H}}_{1}=\sum_{P} \mathcal{H}_{P}\left\{S_{P \alpha}\right\}+\sum_{P, \alpha} H_{P \alpha} S_{P \alpha} .
$$

The minimization is over real values of the $\left\{H_{P \alpha}\right\}$. It may seem curious that the $\left\{\sigma_{P \alpha}\right\}$ do not appear explicitly anywhere. In fact, the functional being minimized on the right hand side of Eq. (A16) is precisely $\mathcal{F}\left\{H_{P \alpha}, \sigma_{P \alpha}\left\{H_{P \alpha}\right\} ; h_{P \alpha}\right\}$ in which the first line of Eq. (A9) has already been substituted for the dependence of the $\left\{\sigma_{P \alpha}\right\}$ on the $\left\{H_{P \alpha}\right\}$. This parametric dependence of the $\left\{\sigma_{P \alpha}\right\}$ on the $\left\{H_{P \alpha}\right\}$ defines a particular trajectory that not only is guaranteed to pass through the saddle point, but for which the saddle point is actually an extremum.

The Bogoliubov procedure often produces an inconsistent free energy. The procedure above is guaranteed not to suffer from this problem. The key ingredient, as we have seen, is that a free minimization parameter $H_{P \alpha}$ should be introduced for each and every single plaquette variable $S_{P \alpha}$ that appears in $\mathcal{A}$. This can actually be seen directly within the Bogoliubov procedure: just as $H_{P \alpha}$ in Eq. (A7) vanishes if the corresponding $\sigma_{P \alpha}$ does not appear in $\mathcal{A}\left\{\sigma_{P \alpha}\right\}$, it is easy to show that the same is true in Eq. (A17). Thus Eq. (A17) is the most general form of $\overline{\mathcal{H}}_{1}(\lambda)$ that one need consider. 


\section{APPENDIX B: FREE ENERGIES: INTERPLAQUETTE CONTRIBUTION}

Using the formalism developed in Appendix A, the free-energy functional corresponding to the tiling shown in Fig. 8 is given by

$$
\begin{aligned}
\mathcal{F}^{(6)}\left\{H_{P \alpha} ; \sigma_{P \alpha}\right\}= & \sum_{P} \Phi^{(6)}\left\{H_{P \alpha}\right\}-\sum_{P \alpha}\left(H_{P \alpha}+h_{P \alpha}\right) \sigma_{P \alpha}-\lambda_{1} K \sum_{P}\left[\sigma_{P 1}\left(\sigma_{Q_{1} 1}+\sigma_{Q_{2} 6}+\sigma_{Q_{5} 4}\right)+\sigma_{P 2}\left(\sigma_{Q_{5} 4}+\sigma_{Q_{5} 2}\right)\right. \\
& \left.+\sigma_{P 3}\left(\sigma_{Q_{2} 3}+\sigma_{Q_{3} 6}\right)+\sigma_{P 4}\left(\sigma_{Q_{4} 5}+\sigma_{Q_{4} 6}+\sigma_{Q_{5} 1}+\sigma_{Q_{5} 2}\right)+\sigma_{P 5}\left(\sigma_{Q_{4} 4}+\sigma_{Q_{4} 5}\right)+\sigma_{P 6}\left(\sigma_{Q_{2} 3}+\sigma_{Q_{2} 1}+\sigma_{Q_{4} 4}\right)\right] \\
& -\lambda_{2} L \sum_{P}\left[\sigma_{P 1}\left(\sigma_{Q_{1} 3}+\sigma_{Q_{2} 3}+\sigma_{Q_{5} 2}\right)+\sigma_{P 2}\left(\sigma_{Q_{2} 6}+\sigma_{Q_{4} 5}+\sigma_{Q_{5} 1}+\sigma_{Q_{5} 5}\right)+\sigma_{P 3}\left(\sigma_{Q_{1} 1}+\sigma_{Q_{2} 5}+\sigma_{Q_{2} 1}\right.\right. \\
& \left.+\sigma_{Q_{4} 4}+\sigma_{Q_{5} 4}\right)+\sigma_{P 4}\left(\sigma_{Q_{4} 4}+\sigma_{Q_{4} 3}+\sigma_{Q_{5} 3}+\sigma_{Q_{5} 4}\right)+\sigma_{P 5}\left(\sigma_{Q_{2} 3}+\sigma_{Q_{4} 2}+\sigma_{Q_{4} 6}+\sigma_{Q_{5} 2}\right) \\
& \left.+\sigma_{P 6}\left(\sigma_{Q_{2} 6}+\sigma_{Q_{2} 2}+\sigma_{Q_{3} 6}+\sigma_{Q_{4} 5}\right)\right]-\lambda_{1} K \sum_{P} \sigma_{P 1} \sigma_{P_{4} 6} \\
& -\lambda_{2} L \sum_{P}\left(\sigma_{P 1} \sigma_{P_{1} 4}+\sigma_{P 1} \sigma_{P_{4} 5}+\sigma_{P 2} \sigma_{P_{4} 6}\right)+(P \rightarrow Q)
\end{aligned}
$$

where we have defined two sublattices, $P$ and $Q$, for the two different plaquette orientations, and the plaquette labels are shown in Fig. 10. The final term, denoted symbolically, is the interaction between plaquettes on the same sublattice $Q$ and takes the same form as the two previous terms. The scale factors, $\lambda_{1}$ and $\lambda_{2}$, have again been introduced. Notice that there is no obvious rotational symmetry to the interactions, and hence that the saddle-point values of the $\sigma_{P \alpha}$ will all be different even in the unreconstructed phases. Note, however, that there is sufficient translational and inversion symmetry that they will be independent of $P$ and $Q$.

Similarly, the free-energy functional corresponding to the distorted lattice tiling shown in Fig. 10 is given by

$$
\begin{aligned}
\mathcal{F}^{(6)}\left\{H_{P \alpha} ; \sigma_{P \alpha}\right\}= & \sum_{P} \Phi^{(6)}\left\{H_{P \alpha}\right\}-\sum_{P \alpha}\left(H_{P \alpha}+h_{P \alpha}\right) \sigma_{P \alpha}-\lambda_{1} K \sum_{P}\left[\sigma_{P 1}\left(\sigma_{Q_{2} 4}+\sigma_{Q_{3} 6}\right)+\sigma_{P 2} \sigma_{Q_{2} 2}+\sigma_{P 3} \sigma_{Q_{3} 3}\right. \\
& \left.+\sigma_{P 4}\left(\sigma_{Q_{2} 1}+\sigma_{Q_{5} 6}\right)+\sigma_{P 5} \sigma_{Q_{5} 5}+\sigma_{P 6}\left(\sigma_{Q_{3} 1}+\sigma_{Q_{5} 4}\right)\right]-\alpha_{1} \sum_{P}\left[\sigma_{P 2}\left(\sigma_{Q_{2} 3}+\sigma_{Q_{2} 5}+\sigma_{Q_{3} 3}+\sigma_{Q_{5} 5}\right)\right. \\
& \left.+\sigma_{P 3}\left(\sigma_{Q_{3} 2}+\sigma_{Q_{3} 5}+\sigma_{Q_{2} 2}+\sigma_{Q_{5} 5}\right)+\sigma_{P 5}\left(\sigma_{Q_{5} 2}+\sigma_{Q_{5} 3}+\sigma_{Q_{2} 2}+\sigma_{Q_{3} 3}\right)\right]-\alpha_{2} \sum_{P}\left[\sigma_{P 1}\left(\sigma_{Q_{2} 5}+\sigma_{Q_{3} 5}\right)\right. \\
& \left.+\sigma_{P 2}\left(\sigma_{Q_{3} 6}+\sigma_{Q_{5} 6}\right)+\sigma_{P 3}\left(\sigma_{Q_{2} 4}+\sigma_{Q_{5} 4}\right)+\sigma_{P 4}\left(\sigma_{Q_{2} 3}+\sigma_{Q_{5} 3}\right)+\sigma_{P 5}\left(\sigma_{Q_{2} 1}+\sigma_{Q_{3} 1}\right)+\sigma_{P 6}\left(\sigma_{Q_{3} 2}+\sigma_{Q_{5} 2}\right)\right] \\
& -\beta \sum_{P}\left[\sigma_{P 1} \sigma_{Q_{1} 1}+\sigma_{P 4} \sigma_{Q_{4} 4}+\sigma_{P 6} \sigma_{Q_{6} 6}\right]-\gamma \sum_{P}\left[\sigma_{P 1}\left(\sigma_{Q_{2} 2}+\sigma_{Q_{3} 3}\right)+\sigma_{P 2}\left(\sigma_{Q_{2} 1}+\sigma_{Q_{2} 4}\right)\right. \\
& +\sigma_{P 3}\left(\sigma_{Q_{3} 1}+\sigma_{Q_{3} 6}\right)+\sigma_{P 4}\left(\sigma_{Q_{2} 2}+\sigma_{Q_{5} 5}\right)+\sigma_{P 5}\left(\sigma_{Q_{5} 4}+\sigma_{Q_{5} 6}\right)+\sigma_{P 6}\left(\sigma_{Q_{3} 3}+\sigma_{Q_{5} 5}\right) \\
& -\frac{1}{2} \delta \sum_{P}\left[\sigma_{P 1}\left(\sigma_{P_{1} 6}+\sigma_{P_{2} 4}\right)+\sigma_{P 4}\left(\sigma_{P_{3} 6}+\sigma P_{5} 1\right)+\sigma_{P 6}\left(\sigma_{P_{4} 4}+\sigma_{P_{6} 1}\right)\right]-\frac{1}{2} \delta(P \rightarrow Q),
\end{aligned}
$$

where the plaquette labels are shown in Fig. 10, and where the final term is again the interaction between plaquettes on the same sublattice $Q$ and takes the same form as the immediately preceding term. We choose the coefficients $\alpha_{1}, \quad \alpha_{2}, \quad \beta, \quad \gamma, \delta$ in order to best mimic the interplaquette interactions on the original undistorted lattice. In order to obtain the same overall interaction between each site and the other plaquettes we require $2 \lambda_{1} K+2 \alpha_{2}+\beta+2 \gamma$ $+2 \delta=4 \lambda_{1} K+5 \lambda_{2} L$ (for the corner sites) and $\lambda_{1} K+4 \alpha_{1}$
$+2 \alpha_{2}+2 \gamma=2 \lambda_{1} K+5 \lambda_{2} L$ (for the edge sites). This also ensures the correct values of $h_{2}^{\text {in }}$ and $h_{2}^{\text {out }}$ quoted below Eq. (2.20). By somewhat arbitrarily matching up the various bonds in Figs. 8 and 10, we take

$$
\alpha_{1}=\alpha_{2}=\frac{1}{2} \lambda_{2} L, \quad \beta=\frac{2}{3} \gamma=\delta=\frac{1}{3}\left(\lambda_{1} K+2 \lambda_{2} L\right) .
$$

Finally specializing to the unreconstructed phases where the $\sigma$ 's take the value $M_{\text {out }}$ on the corner sites and $M_{\text {in }}$ on the 
edge sites, we obtain the free energy

$$
\begin{aligned}
\frac{1}{N} \mathcal{F}^{(6)}\left(H_{\text {in }}, H_{\text {out }} ; M_{\text {in }}, M_{\text {out }}\right) \\
=\frac{1}{6} \Phi^{(6)}\left(H_{\text {in }}, H_{\text {out }}\right)-\frac{1}{2}\left[\left(H_{\text {out }}+h\right) M_{\text {out }}+\left(H_{\text {in }}+h\right) M_{\text {in }}\right] \\
\quad-\left(\lambda_{1} K+\frac{1}{2} \beta+\delta\right) M_{\text {out }}^{2}-\left(\frac{1}{2} \lambda_{1} K+2 \alpha_{1}\right) M_{\text {in }}^{2} \\
\quad-2\left(\alpha_{2}+\gamma\right) M_{\text {out }} M_{\text {in }} .
\end{aligned}
$$

Substituting Eq. (B3) yields the final result (2.21) on which we base our computations.

The seven site plaquette tiling shown in Fig. 9 yields the free energy

$$
\begin{aligned}
\mathcal{F}^{(7)}\left\{H_{P \alpha} ; \sigma_{P \alpha}\right\} & =\sum_{P} \Phi^{(7)}\left\{H_{P \alpha}\right\}-\sum_{P \alpha}\left(H_{P \alpha}+h_{P \alpha}\right) \sigma_{P \alpha} \\
& -\frac{1}{2} \lambda_{1} K \sum_{P}\left[\sigma_{P 1}\left(\sigma_{P_{6} 5}+\sigma_{P_{1} 6}+\sigma_{P_{1} 7}\right)+(\text { five terms })\right. \\
& -\frac{1}{2} \lambda_{2} L \sum_{P}\left[\sigma_{P 1}\left(\sigma_{P_{6} 7}+\sigma_{P_{6} 2}+\sigma_{P_{1} 4}+\sigma_{P_{2} 3}\right)\right. \\
& +(\text { five terms })]-\frac{1}{2} \lambda_{2} L \sum_{P} \sigma_{P 4}\left(\sigma_{P_{1} 7}+\sigma_{P_{2} 6}\right. \\
& \left.+\sigma_{P_{3} 3}+\sigma_{P_{4} 1}+\sigma_{P_{5} 2}+\sigma_{P_{6} 5}\right),
\end{aligned}
$$

where the plaquette labels are shown in Fig. 9. Similarly, the distorted lattice tiling in Fig. 11 yields

$$
\begin{aligned}
& \mathcal{F}^{(7)}\left\{H_{P \alpha} ; \sigma_{P \alpha}\right\} \\
&=\sum_{P} \Phi^{(7)}\left\{H_{P \alpha}\right\}-\sum_{P \alpha}\left(H_{P \alpha}+h_{P \alpha}\right) \sigma_{P \alpha} \\
& \quad-\frac{1}{2} \lambda_{1} K \sum_{P}\left[\sigma_{P 1}\left(\sigma_{P_{6} 5}+\sigma_{P_{1} 6}\right)+(\text { five terms })\right] \\
&-\frac{1}{2} \alpha \sum_{P}\left[\sigma_{P 1}\left(\sigma_{P_{6} 7}+\sigma_{P_{1} 7}\right)+(\text { five terms })\right] \\
&-\frac{1}{2} \beta \sum_{P}\left[\sigma_{P 1}\left(\sigma_{P_{6} 2}+\sigma_{P_{1} 3}+\sigma_{P_{2} 3}+\sigma_{P_{5} 2}\right)\right. \\
&+(\text { five terms })]-\frac{1}{2} \gamma \sum_{P}\left[\sigma_{P_{1}}\left(\sigma_{P_{6} 4}+\sigma_{P_{1} 4}\right)\right. \\
&\left.+(\text { five terms })+\sigma_{P 4}\left(\sigma_{P_{6} 5}+\sigma_{P_{1} 6}+\text { ten terms }\right)\right] .
\end{aligned}
$$

In order to best match the overall interactions between a given site and other plaquettes in Eqs. (B5) and (B6) we choose

$$
\alpha=\frac{1}{2}\left(\lambda_{1} K+\lambda_{2} L\right), \quad \beta=\gamma=\frac{1}{2} \lambda_{2} L
$$

Specializing to unreconstructed phases the $\sigma$ 's take the value $M_{\text {out }}$ on the outer ring of six sites and the value $M_{\text {in }}$ on the central site, we obtain

$$
\begin{aligned}
\frac{1}{N} \mathcal{F}^{(7)}\left(H_{\text {in }}, H_{\text {out }} ; M_{\text {in }}, M_{\text {out }}\right) & \\
= & \frac{1}{7} \Phi^{(7)}\left(H_{\text {in }}, H_{\text {out }}\right)-\frac{1}{7}\left[6\left(H_{\text {out }}+h\right) M_{\text {out }}+\left(H_{\text {in }}+h\right) M_{\text {in }}\right] \\
& \quad-\frac{1}{7}\left(6 \lambda_{1} K+6 \alpha+12 \beta\right) M_{\text {out }}^{2}-\frac{12}{7} \gamma M_{\text {out }} M_{\text {in }} .
\end{aligned}
$$

Substituting Eq. (B7) yields the final result, Eq. (2.23), on which we base our computations. In fact, since the tiling in Fig. 9 (unlike that in Fig. 8) retains the rotational symmetry of the plaquette, one may also simplify Eq. (B5) using $M_{\text {out }}$ and $M_{\text {in }}$. The result is in fact identical to Eq. (2.23), which further supports the choice of parameters, Eq. (B7).

\section{APPENDIX C: FREE ENERGIES: INTRAPLAQUETTE CONTRIBUTION}

In this appendix we perform the trace over internal plaquette variables required to compute $\Phi^{(p)}$. This computation is completely independent of previous considerations about how to embed the plaquette in the full lattice. In addition, we may now account properly for the RSOS constraint simply by restricting the trace to those configurations that respect it.

We begin with the simplest spin-1 model on the square lattice, Eq. (2.13) [Fig. 6(a)], and the two sublattice magnetizations, Eq. (2.12). To obtain the free energy we need only sum over all possible configurations of the four spins. The RSOS condition implies that spins +1 and -1 cannot be nearest neighbors. There are 21 energetically distinct allowed spin configurations and we obtain

$$
\begin{aligned}
\Phi^{(4)}\left(H_{A}, H_{B}\right) & =-\ln \left[\operatorname{tr}\left(e^{-\overline{\mathcal{H}}_{0}^{(4)}-H_{A}\left(s_{1}+s_{3}\right)-H_{B}\left(s_{2}+s_{4}\right)}\right)\right], \\
& =-\ln \left[\sum_{m=0}^{2} \sum_{n=0}^{2} a_{m n} \cosh \left(m H_{A}+n H_{B}\right)\right],
\end{aligned}
$$

where, to simplify notation, we define

$$
x=e^{-1 / 2 L}, \quad y=e^{-1 / 2 K}, \quad z=e^{-\widetilde{h}_{2}},
$$

where $\widetilde{h_{2}}=h_{2}+\lambda_{1} K+\frac{3}{2} \lambda_{2} L$. In terms of these variables,

$$
\begin{gathered}
a_{00}=1+4 x^{4} y^{4} z^{2}, \quad a_{01}=a_{10}=4 x y^{2} z \\
a_{02}=a_{20}=2 y^{4} z^{2}, \quad a_{11}=8 x^{2} y^{2} z^{2}, \\
a_{12}=a_{21}=4 x y^{2} z^{3}, \quad a_{22}=2 z^{4} .
\end{gathered}
$$

These equations, in conjunction with the saddle-point conditions (2.14) and (2.15), completely determine the thermodynamics of the model. Note that all spins are clearly equivalent for this plaquette and we need not worry about inner and outer values of $H$ and $M$ as we did for the triangular lattice [see Eqs. (2.21) and (2.23) and below]. To describe the unreconstructed phases we may take $H_{A}=H_{B} \equiv H$. Equation (C1) then simplifies to 


$$
\Phi^{(4)}(H)=-\ln \left[\sum_{m=0}^{4} a_{m} \cosh (m H)\right]
$$

where

$$
\begin{gathered}
a_{0}=1+4 x^{4} y^{4} z^{2}, \quad a_{1}=8 x y^{2} z \\
a_{2}=8 x^{2} y^{2} z^{2}+4 y^{4} z^{2}, \quad a_{3}=8 x y^{2} z^{3}, \quad a_{4}=2 z^{4} .
\end{gathered}
$$

For the six spin triangular lattice plaquette [Fig. 6(b)] we consider only unreconstructed phases. As discussed in Sec. II C 2 and in Appendix B we still need to keep two fields, $H_{\text {out }}$ and $H_{\text {in }}$. There are 47 energetically distinct allowed spin configurations and we obtain then

$$
\begin{aligned}
\Phi^{(6)}( & \left.H_{\text {in }}, H_{\text {out }}\right) \\
= & -\ln \left(\operatorname { t r } \left\{\operatorname { e x p } \left[-\overline{\mathcal{H}}_{0}^{(6)}-H_{\text {out }}\left(s_{1}+s_{4}+s_{6}\right)\right.\right.\right. \\
& \left.\left.\left.-H_{\text {in }}\left(s_{2}+s_{3}+s_{5}\right)\right]\right\}\right) \\
= & -\ln \left[\sum_{m=0}^{3} \sum_{n=-1}^{3} a_{m n} \cosh \left(m H_{\text {out }}+n H_{\text {in }}\right)\right] .
\end{aligned}
$$

Defining $x$ and $y$ as in Eq. (C2), and

$$
z_{o}=e^{-\widetilde{h}_{2}^{\text {out }}}, \quad z_{i}=e^{-\widetilde{h}_{2}^{\text {in }},}
$$

where $\widetilde{h}_{2}^{\text {out }}=h_{2}+2 \lambda_{1} K+\frac{5}{2} \lambda_{2} L$ and $\widetilde{h_{2}^{\text {in }}}=h_{2}+\lambda_{1} K+\frac{5}{2} \lambda_{2} L$, the nonvanishing $a_{m n}$ are

$$
\begin{gathered}
a_{00}=1+6 x^{2} y^{4} z_{o}^{2}, \quad a_{10}=6 x y^{2} z_{o}+6 x^{3} y^{6} z_{o}^{3}, \\
a_{01}=6 x y^{4} z_{i}+12 x^{5} y^{6} z_{o} z_{i}, \quad a_{20}=6 x^{2} y^{4} z_{o}^{2}, \\
a_{02}=6 x^{2} y^{6} z_{i}^{2}, \quad a_{11}=12 x^{2} y^{4} z_{o} z_{i}+6 y^{6} z_{o} z_{i}, \\
a_{1,-1}=6 x^{4} y^{6} z_{o} z_{i}, \quad a_{30}=2 x^{3} y^{6} z_{o}^{3}, \quad a_{03}=2 x^{3} y^{6} z_{i}^{3}, \\
a_{21}=6 x^{3} y^{4} z_{o}^{2} z_{i}+12 x y^{6} z_{o}^{2} x_{i}, \\
a_{12}=6 x^{3} y^{4} z_{o} z_{i}^{2}+12 x y^{6} z_{o} z_{i}^{2}, \\
a_{31}=6 x^{2} y^{6} z_{0}^{3} z_{i}, \quad a_{13}=6 x^{2} y^{4} z_{o} z_{i}^{3}, \\
a_{22}=12 x^{2} y^{4} z_{o}^{2} z_{i}^{2}+6 y^{6} z_{o}^{2} z_{i}^{2}, \quad a_{32}=6 x y^{4} z_{o}^{3} z_{i}^{2}, \\
a_{23}=6 x y^{2} z_{o}^{2} z_{i}^{3}, \quad a_{33}=2 z_{o}^{3} z_{i}^{3} .
\end{gathered}
$$

For the seven spin plaquette [Fig. 6(c)] there are 63 energetically distinct allowed spin configurations, and we obtain

$$
\begin{aligned}
\Phi^{(7)}( & \left.H_{\text {in }}, H_{\text {out }}\right) \\
= & -\ln \left(\operatorname { t r } \left\{\operatorname { e x p } \left[-\overline{\mathcal{H}}_{0}^{(6)}-H_{\text {out }}\left(s_{1}+s_{2}+s_{3}+s_{5}+s_{6}+s_{7}\right)\right.\right.\right. \\
& \left.\left.\left.-H_{\mathrm{in}} s_{4}\right]\right\}\right) \\
= & -\ln \left[\sum_{m=0}^{6} \sum_{n=0}^{1} a_{m n} \cosh \left(m H_{\text {out }}+n H_{\text {in }}\right)\right]
\end{aligned}
$$

Using Eqs. (C2) amd (C7), where now $\widetilde{h}_{2}^{\text {out }}=h_{2}+\frac{3}{2} \lambda_{1} K$ $+2 \lambda_{2} L$ and $\widetilde{h_{2}^{\text {in }}}=h_{2}+3 \lambda_{2} L$, the nonvanishing $a_{m n}$ are

$$
\begin{gathered}
a_{00}=1+12 x^{6} y^{6} z_{o}^{2}+6 x^{4} y^{6} z_{o}^{2}+6 x^{12} y^{8} z_{o}^{4}, \quad a_{01}=2 y^{6} z_{i} \\
a_{10}=12 x^{2} y^{3} z_{o}+24 x y^{8} z_{o}^{3}+12 x^{8} y^{9} z_{o}^{3}, \quad a_{11}=2 x^{2} y^{7} z_{o} z_{i} \\
a_{20}=12 x^{4} y^{4} z_{o}^{2}+12 x^{2} y^{6} z_{o}^{3}+6 x^{4} y^{6} z_{o}^{2}+12 x^{10} y^{8} z_{o}^{4} \\
a_{21}=12 x^{4} y^{6} z_{o}^{2} z_{i}+12 x^{2} y^{8} z_{o}^{2} z_{i}+6 x^{4} y^{8} z_{o}^{2} z_{i} \\
a_{30}=12 x^{4} y^{5} z_{o}^{3}+24 x^{4} y^{7} z_{o}^{3} \\
a_{31}=12 x^{4} y^{5} z_{o}^{3} z_{i}+24 x^{4} y^{7} z_{o}^{3} z_{i}+4 y^{9} z_{o}^{3} \\
a_{40}=12 x^{4} y^{6} z_{o}^{4}+12 x^{2} y^{8} z_{o}^{4}+6 x^{4} y^{8} z_{o}^{4} \\
a_{41}=12 x^{4} y^{4} z_{o}^{4} z_{i}+12 x^{2} y^{6} z_{0}^{4} z_{i}+6 x^{4} y^{6} z_{o}^{4} z_{i} \\
a_{50}=12 x^{2} y^{7} z_{o}^{5}, \quad a_{51}=12 x^{2} y^{3} z_{o}^{5} z_{i} \\
a_{60}=2 y^{6} z_{0}^{6}, \quad a_{61}=2 z_{o}^{6} z_{i}
\end{gathered}
$$

\section{APPENDIX D: AN EXPLICIT RSOS MODEL WITH A $\theta$ DOF PHASE}

In Sec. II we observed very generally that in a model with renormalized parameter $u_{R}<0$, when $y_{R}$ changes sign, one expects to see a $\theta$ DOF phase, characterized by a continuously varying upper layer coverage. However, none of the solid-on-solid models we have treated show this phase. In this appendix we outline briefly the derivation of a more complicated RSOS model which does contain a $\theta$ DOF phase.

The basic steps in the derivation are as follows. We first introduce the staggered eight-vertex $(8 \mathrm{~V})$ model, which is then shown to be equivalent to a staggered body-centered cubic solid-on-solid (BCSOS) model. ${ }^{29}$ The free energy of the staggered $8 \mathrm{~V}$ model is invariant under a certain symmetry operation and this fact is then used to obtain a more convenient $8 \mathrm{~V}$ model. We then show that this modified $8 \mathrm{~V}$ model is exactly mappable to a system of interpenetrating Ising spins with four-spin interactions. Finally we show that the isotropic Ashkin-Teller (AT) model $^{24}$ can be mapped onto exactly the same Ising system. The AT model contains a $\theta \mathrm{DOF}$ phase and this sequence of mappings then produces a BCSOS model with this phase and a corresponding intermeshed layering phase diagram.

\section{Vertex models}

The $8 \mathrm{~V}$ models are defined on a square lattice with $d i$ rected bonds between sites. Each site is constrained to have an even number of bonds going in or out of it. This leads to six types of vertex with two bonds in and two bonds out, one type with four bonds in, and one type with four bonds out, for a total of eight. Each vertex type has an associated Boltzmann weight, $w_{i}=e^{-\beta \epsilon_{i}}>0, i=1, \ldots, 8$. If the last two are given zero weight one obtains the six-vertex $(6 \mathrm{~V})$ model. ${ }^{30}$ The latter have a direct mapping to an RSOS model with the additional condition that any two neighboring plaquettes have a unit height difference. Thus each directed line is associated with a surface step (up to the right, down to the left), and a unique correspondence can be made with any 


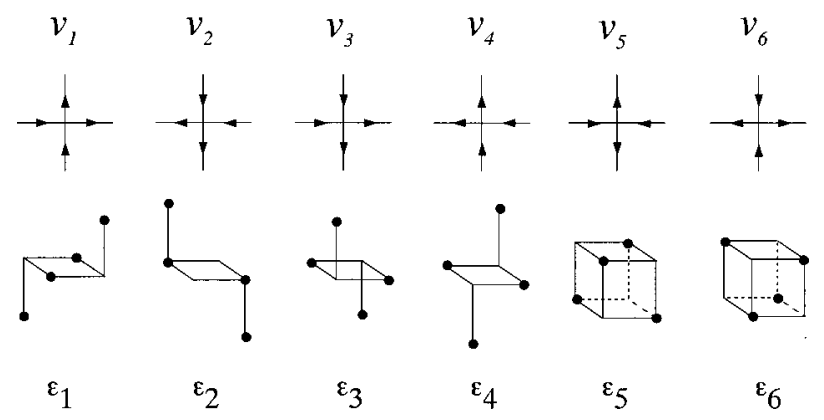

FIG. 21. The six-vertex model. Shown below each vertex is a schematic of the corresponding surface configuration.

pattern of bonds and a set of surface heights (see Fig. 21). Since nearest-neighbor heights always differ by one, the heights on each of the two antiferromagnetic sublattices all have the same parity: one sublattice will have all even heights, the other all odd. One may conveniently identify this with the (100) surface of a body-centered-cubic structure, and this mapping of the $6 \mathrm{~V}$ model, therefore, defines the BCSOS model of the surface.

The $6 \mathrm{~V}$ model can be solved exactly when the additional symmetries $w_{1}=w_{2} \equiv a, w_{3}=w_{4} \equiv b$, and $w_{5}=w_{6} \equiv c$ are imposed. ${ }^{31}$ The model shows a Kosterlitz-Thouless roughening transition on the line $a+b=c$ between a checkerboard ordered phase $(a+b<c)$ and a rough phase $(a+b>c)$. If the last pair of vertices, with weights $w_{7}=w_{8} \equiv d$, are included (see Fig. 22), there is no longer a consistent mapping to a set of surface height models, but exact solubility is maintained in certain subspaces. ${ }^{32}$ Although in our applications to roughening we shall always set $d=0$, it is useful to carry it along more generally until the end.

We shall require a further generalization of these models. Since the two antiferromagnetic sublattices now correspond to two different sets of (100) planes in a bcc structure, a natural generalization is to a two-component model with the two sets of planes composed of two different species of atoms, $A$ and $B$, forming a $\mathrm{NaCl}$ type of structure. As shown in Fig. 23 the $6 \mathrm{~V}$ model now generalizes to a staggered $\mathrm{BCSOS}$ model with 12 vertices. As shown, we will still consider only a three-parameter subspace of these models. The staggered $8 \mathrm{~V}$ model is defined similarly. As stated, it will be formally useful to carry along the four extra staggered $8 \mathrm{~V}$ model vertices as well, all with weight $d$ that will vanish in the end.

\section{Equivalence of staggered BCSOS and AT models}

In an obvious notation, let $Z(a, b, c, d \mid b, a, c, d)$ be the partition function of the class of staggered $8 \mathrm{~V}$ models defined above. In order to map this model onto the AT model we will need the identity

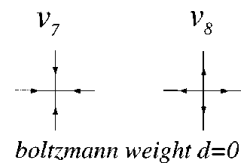

FIG. 22. Vertices included (with, in the end, zero weight) to allow the $6 \mathrm{~V}$ model to be considered as a special case of the $8 \mathrm{~V}$ model.

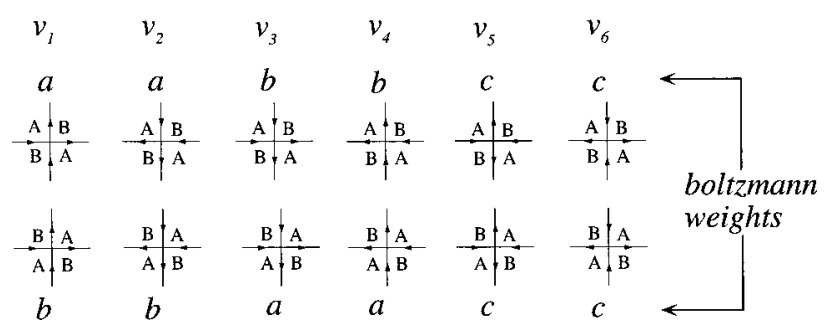

FIG. 23. The 12 distinct vertices in the two-component BCSOS model along with their Boltzmann weights.

$$
Z(a, b, c, d \mid b, a, c, d)=Z(c, d, a, b \mid c, d, b, a) .
$$

To see Eq. (D1) simply note that the vertices of the $8 \mathrm{~V}$ model can be thought of as lying in one of two sublattices $L_{1}$ and $L_{2}$. For a given configuration, on any bond of the lattice, reverse the direction of the arrow only if the edge is horizontal (vertical) and there is a site of sublattice $L_{2}$ immediately to the right (bottom) of the edge. If the vertices are labeled by $v_{i},(i=1, \ldots, 8)$ then under this transformation: $v_{1} \leftrightarrow v_{5}$, $v_{2} \leftrightarrow v_{6}, v_{3} \leftrightarrow v_{7}$, and $v_{4} \leftrightarrow v_{8}$ on both sublattices and we have generated a new configuration. The weight of each configuration of the original model can be thought of as the weight of this resultant configuration in a different $8 \mathrm{~V}$ model and this immediately yields Eq. (D1).

An Ising spin model with four-spin interactions is now associated with the staggered $8 \mathrm{~V}$ model introduced above as follows. On each plaquette (of either $A$ or $B$ type) of this $8 \mathrm{~V}$ model we place an Ising spin and assume that spin on one special site $S_{0}$ is fixed so that it can only point up. A correspondence between the $8 \mathrm{~V}$ configurations and the spin configurations is established. If the arrow on an edge points to the right or up (left or down) then the product of the spins on either side of the edge is $+1(-1)$. We see that for each arrow configuration there is a unique spin configuration when we fix the one spin. Thus there is a clear mapping to an Ising spin system where interactions around a vertex (i.e., four-spin interactions) are allowed. It is then easy to see that if we consider a Hamiltonian of the form

$$
\mathcal{H}=-P \sum_{\langle i j\rangle \in A} s_{i_{A}} s_{j_{A}}-Q \sum_{\langle k l\rangle \in B} s_{k_{B}} s_{l_{B}}-R \sum_{V} s_{i_{A}} s_{j_{A}} s_{k_{B}} s_{l_{B}}
$$

where the first two sums are over nearest neighbors on the $A$ and $B$ sublattices and the last sum is over plaquettes, then the correspondences

$$
\begin{gathered}
c=\exp (P+Q+R), \quad d=\exp (-P-Q+R), \\
b=\exp (-P+Q-R), \quad a=\exp (Q-P-R)
\end{gathered}
$$

[substituted into the right-hand side of Eq. (D1); the mapping produces an $8 \mathrm{~V}$ model with nonzero $d$, and it is here that the symmetry (D1) is required to produce the required $6 \mathrm{~V}$ 
model] produces precisely the same Boltzmann weights for the two models. Freeing the constraint on the one special spin we have $Z_{\text {spin }}=2 Z_{\text {staggered } 8 \mathrm{~V}}$. Thus the staggered $8 \mathrm{~V}$ model is isomorphic to this Ising model.

Now, the AT model is defined on a simple square lattice. On each lattice site $i$ there are two Ising spins, $s_{i}$ and $\sigma_{i}$. Spins on nearest-neighbor sites are coupled by two- and four-spin interactions:

$$
\mathcal{H}_{\mathrm{AT}}=-\sum_{\langle i j\rangle}\left(K s_{i} s_{j}+K \sigma_{i} \sigma_{j}+K_{4} s_{i} s_{j} \sigma_{i} \sigma_{j}\right) .
$$

This defines the isotropic AT model (more generally the two $K$ 's could have been different; an extreme anisotropic limit maps onto a certain one-dimensional quantum problem. ${ }^{25}$ ) If a duality transformation is performed on one set of spins (say the $\sigma$ spins; see Ref. 32 for details) then the AT model can be expressed as a system of two interpenetrating square Ising lattices with four-spin interactions, precisely as above, with the same Hamiltonian (D2). The relation between $P, Q$, and $R$ of Eq. (D2) and $K$ and $K_{4}$ of Eq. (D4) is

$$
\begin{gathered}
\exp (P+Q+R)=e^{2 K+K_{4}}\left(1+e^{-4 K}\right) / \sqrt{2}, \\
\exp (-P-Q+R)=0, \\
\exp (-P+Q-R)=\sqrt{2} e^{2 K+K_{4}} e^{-2\left(K+K_{4}\right)}, \\
\exp (Q-P-R)=e^{2 K+K_{4}}\left(1-e^{-4 K}\right) / \sqrt{2} .
\end{gathered}
$$

This completes the mapping of the BCSOS model onto the isotropic AT model. The AT model has been studied extensively. Its full phase diagram can be found in Ref. 32. If we define $a=e^{-J_{A}}, b=e^{-J_{B}}$, normalize $c=1$ and take $d$ $=0$ we then find

$$
J_{A}=2 K_{4}+\ln \cosh (2 K), \quad J_{B}=-\ln \tanh (2 K) .
$$

The phase diagram of the staggered BCSOS model can then be mapped out in terms of $J_{A}$ and $J_{B}$; the relevant portion of it is shown in Fig. 24. There is a line of continuously varying

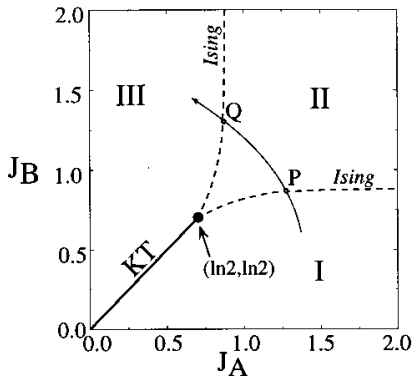

(a)

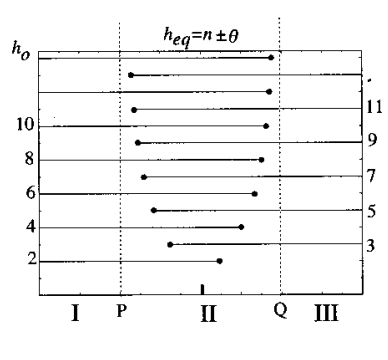

(b)
FIG. 24. Staggered BCSOS model phase diagram with $a$ $\equiv e^{-J_{A}}, \quad b \equiv e^{-J_{B}}$, and $c=1$. The line labeled PQ is a path through phase space that would yield the layering diagram shown in Fig. 18 with region I corresponding to $y<-y_{R}^{c}$, region II to $-y_{R}^{c}<y_{R}$ $<y_{R}^{c}$, and region III to $y>y_{R}^{c}$.

exponents that splits into two Ising lines. In region I the average height of the surface is an even integer and the $A$ sublattice is essentially completely ordered while the $B$ lattice is disordered with about half the $B$ atoms at a height one layer above the $A$ lattice height and half of the $B$ atoms one layer below. In region III one has the complementary situation in which the $B$ lattice is ordered and the $A$ lattice is disordered. In region II symmetry breaking occurs, and the average column height either increases or decreases continuously, interpolating between the phases in regions II and III. Thus if, as the temperature is varied, the system follows the path $P Q$ shown in the figure, then there will be two Ising transitions with continuous surface height growth occurring between them. When, in addition, a substrate potential is present and the full chemical potential versus temperature phase diagram is mapped out, it will be as shown in Fig. $1(\mathrm{~d})$. It should be noted that the filling factor of the top layer $\theta$ ranges continuously from 0 to 1 (rather than from 0 to half as in previous sections) because we have chosen to define one unit of height as one layer of $A$ atoms or one layer of $B$ atoms instead of as being the sum of one layer of each.
${ }^{1}$ J. V. Jose, L. P. Kadanoff, S. Kirkpatrick, and D. R. Nelson, Phys. Rev. B 16, 1217 (1977).

${ }^{2}$ K. Rommelse and M. den Nijs, Phys. Rev. Lett. 59, 2578 (1987).

${ }^{3}$ M. den Nijs and K. Rommelse, Phys. Rev. B 40, 4709 (1989).

${ }^{4}$ M. den Nijs, Phys. Rev. Lett. 64, 435 (1990).

${ }^{5}$ M. den Nijs, in Phase Transitions in Surface Films 2, edited by H. Taub et al. (Plenum, New York, 1992).

${ }^{6}$ P. B. Weichman and A. Prasad, Phys. Rev. Lett. 76, 2322 (1996).

${ }^{7}$ J. Villain and M. Gordon, Surf. Sci. 125, 1 (1983); V. L. Pokrovsky and A. L. Talapov, in Soviet Scientific Reviews (Academic, New York, 1988), Vol. 1; D. A. Huse and M. E. Fisher, Phys. Rev. B 29, 239 (1984); P. Bak, Rep. Prog. Phys. 45, 587 (1982)

${ }^{8}$ D. R. Nelson and B. I. Halperin, Phys. Rev. B 19, 2457 (1979); A. P. Young, Phys. Rev. B 19, 1855 (1979); K. J. Strandburg, Rev. Mod. Phys. 60, 161 (1988).

${ }^{9}$ H. Taub et al., Phys. Rev. B 16, 455 (1978); C. Tiby and H. Lauter, Surf. Sci. 117, 277 (1982); T. T. Chung, ibid. 87, 348 (1979); Y. Larher, ibid. 87, 348 (1979); J. P. McTague, J. Als-
Nielsen, J. Bohr, and M. Nielsen, Phys. Rev. B 25, 7765 (1982); D. M. Butler, J. A. Litzinger, G. A. Stewart, and R. B. Griffiths, Phys. Rev. Lett. 42, 1289 (1979); E. D. Specht et al., Z. Phys. B 69, 347 (1987).

${ }^{10}$ Notable early experimental work includes A. Thomy and X. Duval, J. Chim. Phys. Phys.-Chim. Biol. 66, 1966 (1969); A. Thomy and X. Duval, ibid. 67, 286 (1970), where layer-by-layer film growth of xenon and krypton on graphite was observed at low temperatures. For further references see Ref. 11 below.

${ }^{11}$ H. S. Youn and G. B. Hess, Phys. Rev. Lett. 64, 918 (1990); H. S. Youn, X. F. Meng, and G. B. Hess, Phys. Rev. B 48, 14556 (1993).

${ }^{12}$ P. Day, M. Lysek, M. LaMadrid, and D. Goodstein, Phys. Rev. B 47, 10716 (1993).

${ }^{13}$ P. Day, M. LaMadrid, M. Lysek, and D. Goodstein, Phys. Rev. B 47, 7501 (1993).

${ }^{14}$ P. Day, Ph.D. thesis, California University of Technology, 1993.

${ }^{15}$ F. Rieutord, R. Simon, R. Conradt, and R. Müeller-Buschbaum, Europhys. Lett. 37, 565 (1997). 
${ }^{16}$ D. Huse, Phys. Rev. B 30, 1371 (1984).

${ }^{17}$ P. B. Weichman, P. Day, and D. Goodstein, Phys. Rev. Lett. 74, 418 (1995).

${ }^{18}$ J. M. Phillips, Q. M. Zhang, and J. Z. Larese, Phys. Rev. Lett. 71, 2971 (1993). See also J. M. Phillips and J. Z. Larese, ibid. 75, 4330 (1995).

${ }^{19}$ More realistic RSOS models that account for such nontrivial lattice structure have appeared in the literature. See, e.g., S. Prestipino, G. Santoro, and E. Tosatti, Phys. Rev. Lett. 75, 4468 (1995); G. Santoro, M. Vendruscolo, S. Prestipino, and E. Tosatti, Phys. Rev. B 53, 13169 (1996); D. L. Woodraska and J. A. Jaszczak, Phys. Rev. Lett. 78, 258 (1997).

${ }^{20}$ We note in passing that more general forms of $v(h)$ were used in early work to investigate the interplay between layering and wetting phenomena: see, e.g., M. P. Nightingale, W. F. Saam, and M. Schick, Phys. Rev. B 30, 3830 (1984), and references therein. In our model the surface is wet at all temperatures. On the other hand, if, for example, a constant term of appropriate sign is added to $v(h)$ one finds a regime in which the film retains a finite thickness at low temperatures even at coexistence, $\Delta \mu \rightarrow 0$, i.e., the surface is nonwet at low temperatures. Layering behavior, induced by entropic effects, is then observed only after a first-order wetting line $T_{w}(\Delta \mu)$ is encountered. If wetting at coexistence occurs below the roughening transition, $T_{w}(0)$ $<T_{r}$, then first-order layering lines emanate from the wetting line at a sequence of triple points [thus $T_{w}(\Delta \mu)$ plays the role of the $\Delta \mu$ axis at $T=0$ in Fig. 1(a)]. If $T_{w}(0)>T_{r}$ the layering lines retract into $T_{w}(\Delta \mu)$ and disappear. In principle, one could include all of these effects into our theory and explore the interplay between wetting and all of the phase diagrams shown in Fig. 1. We shall not pursue this avenue here, however.

${ }^{21}$ Generally, the rigorous statement is that if the $p$ th one is the first nonvanishing Fourier component, the surface will be rough for
$K_{R}=\lim _{l \rightarrow \infty} K(l)<\pi p^{2} / 2$. Generically this will only happen if one has $p-1$ control variables to tune.

${ }^{22}$ The power-law approaches of the layering line endpoints, Eq. (3.15), to the bulk transition temperature presumably have logarithmic corrections at this marginality point for $u$. We have not tried to compute these explicitly.

${ }^{23}$ J. Ashkin and E. Teller, Phys. Rev. 64, 178 (1943).

${ }^{24}$ M. Kohmoto, M. den Nijs, and L. P. Kadanoff, Phys. Rev. B 24, 5229 (1981).

${ }^{25}$ H. J. F. Knops and L. W. J. den Ouden, Ann. Phys. (Leipzig) 138, 155 (1982).

${ }^{26}$ L. P. Kadanoff and A. C. Brown, Ann. Phys. (Leipzig) 121, 318 (1979).

${ }^{27}$ P. B. Weichman and D. Goodstein, Phys. Rev. Lett. 75, 4331 (1995).

${ }^{28}$ J. Zinn-Justin, Quantum Field Theory and Critical Phenomena (Oxford University Press, New York, 1989), Chap. 21 and Appendix 21.

${ }^{29}$ This model was first introduced in a different context by $\mathrm{H}$. Knops, Phys. Rev. B 20, 4670 (1979). See also G. Mazzeo, E. Carlon, and H. van Beijeren, Phys. Rev. Lett. 74, 1391 (1995) for a more recent discussion of certain aspects of its phase diagram.

${ }^{30}$ These are used to model a variety of systems, including ice [J. C. Slater, J. Chem. Phys. 9, 16 (1941)], hence the alternate name ice-type models.

${ }^{31}$ The even more restricted $F$ model in which $a=b<1$ and $c=1$ was first solved by Lieb: see E. H. Lieb, Phys. Rev. 162, 162 (1967); Phys. Rev. Lett. 18, 1046 (1967); 19, 108 (1967). The model for general $a, b, c$ was then solved subsequently: B. Sutherland, Phys. Rev. Lett. 19, 103 (1967); C. N. Yang, ibid. 19, 586 (1967).

${ }^{32}$ See R. J. Baxter, Exactly Solved Models in Statistical Mechanics (Academic Press, London, 1982). 